\title{
Harnessing the Phytotherapeutic Treasure Troves of the Ancient Medicinal Plant Azadirachta indica (Neem) and Associated Endophytic Microorganisms
}

Authors

Ravindra N. Kharwar ${ }^{1}$, Vijay K. Sharma ${ }^{1,2}$, Ashish Mishra ${ }^{1}$, Jitendra Kumar ${ }^{1,2}$, Dheeraj K. Singh ${ }^{1 \#, ~ S a t i s h ~ K . ~ V e r m a ~}{ }^{1}$,

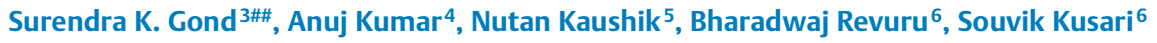

Affiliations

1 Mycopathology and Microbial Technology Laboratory, Department of Botany, Institute of Science, Banaras Hindu University, Varanasi, India

2 Medical School of Kunming University of Science and Technology, Kunming, P. R. China

3 Department of Botany, Visva-Bharati, Shantiniketan, India

4 Department of Botany, Buddha PG College, Kushinagar, India

5 Amity Food and Agriculture Foundation, Amity University, Noida, India

6 Institute of Environmental Research (INFU), Department of Chemistry and Chemical Biology, Technische Universität Dortmund, Dortmund, Germany

Key words neem, Azadirachta indica, Meliaceae, endophytic fungi, actinomycetes, bioactive compounds

received

accepted after revision

published online

Bibliography

Planta Med 2020; 86: 906-940

DOI 10.1055/a-1107-9370

ISSN 0032-0943

(c) 2020. Thieme. All rights reserved.

Georg Thieme Verlag KG, Rüdigerstraße 14, 70469 Stuttgart, Germany

Correspondence

Prof. Dr. Ravindra Nath Kharwar

Mycopathology and Microbial Technology Laboratory,

Department of Botany, Institute of Science, Banaras Hindu

University

Varanasi 221005, Uttar Pradesh, India

Phone: + 9194152900 01, Fax: + 915422368174

rnkharwar@gmail.com
Correspondence

Priv.-Doz. Dr. Souvik Kusari

Institute of Environmental Research (INFU), Department of

Chemistry and Chemical Biology, Technische Universität

Dortmund

Otto-Hahn-Straße 6, 44221 Dortmund, Germany

Phone: + 49(0) 23175540 86, Fax: + 49(0) 2317554084

souvik.kusari@tu-dortmund.de

\section{ABSTRACT}

Azadirachta indica, commonly known as neem, is an evergreen tree of the tropics and sub-tropics native to the Indian subcontinent with demonstrated ethnomedicinal value and importance in agriculture as well as in the pharmaceutical industry. This ancient medicinal tree, often called the "wonder tree", is regarded as a chemical factory of diverse and complex compounds with a plethora of structural scaffolds that is very difficult to mimic by chemical synthesis. Such multifaceted chemical diversity leads to a fantastic repertoire of functional traits, encompassing a wide variety of biological activity and unique modes of action against specific and generalist pathogens and pests. Until now, more than 400 compounds have been isolated from different parts of neem including important bioactive secondary metabolites such as azadirachtin, nimbidin, nimbin, nimbolide, gedunin, and many more. In addition to its insecticidal property, the plant is also known for antimicrobial, antimalarial, antiviral, anti-inflammatory, analgesic, antipyretic, hypoglycaemic, antiulcer, antifertility, anticarcinogenic, hepatoprotective, antioxidant, anxiolytic, molluscicidal, acaricidal, and antifilarial properties. Notwithstanding the chemical and biological virtuosity of neem, it has also been extensively explored for associated microorganisms, especially a class of mutualists called endophytic microorganisms (or endophytes). More than 30 compounds, including neem "mimetic" compounds, have been reported from endophytes harbored in the neem trees in different ecological niches. In this review, we provide an informative and in-depth overview of the topic that can serve as a point of reference for

\# Present Address: Department of Botany, Harish Chandra PG College, Varanasi, India

\#\# Present address: Department of Botany, MMV, Banaras Hindu University, Varanasi, India 
an understanding of the functions and applications of a medicinal plant such as neem, including associated endophytes, within the overall theme of phytopathology. Our review further exemplifies the already-noted current surge of interest in plant and microbial natural products for implications both within the ecological and clinical settings, for a more secure and sustainable future.

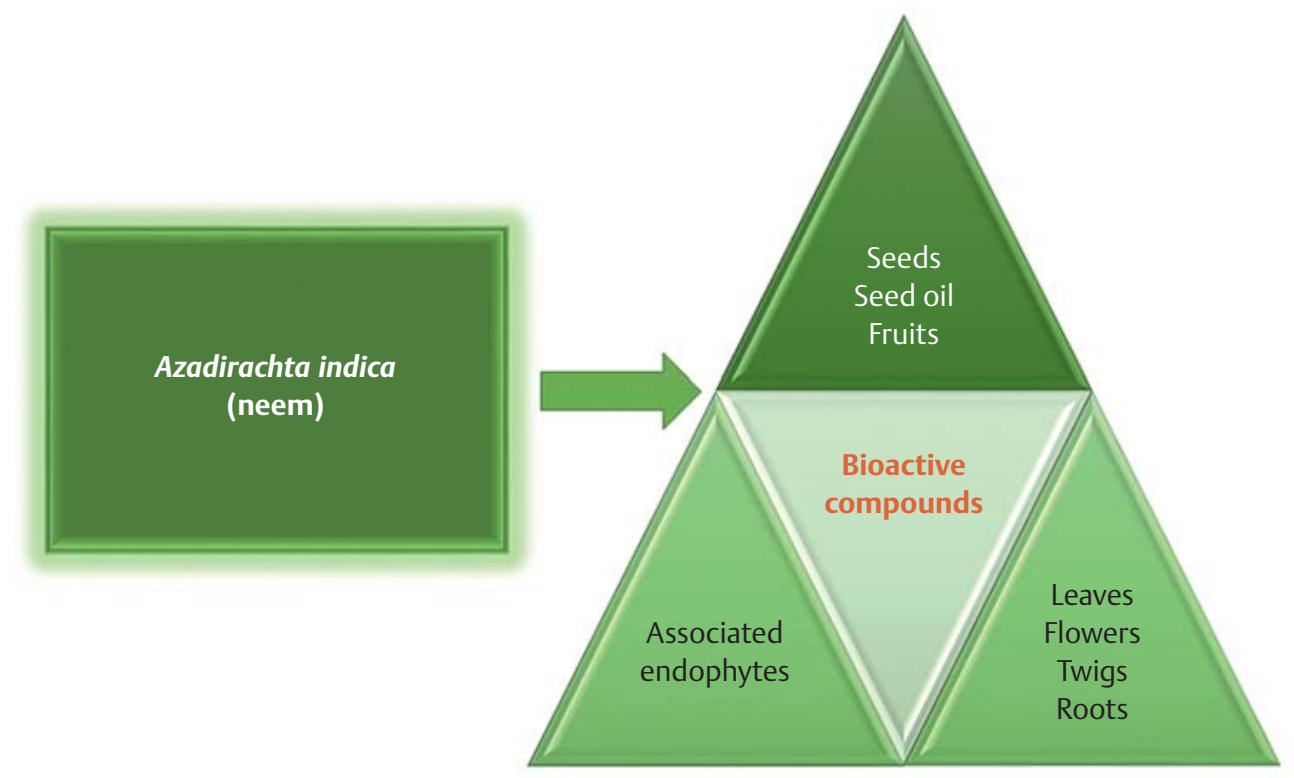

\section{Introduction}

Neem (Azadirachta indica A. Juss.) is native to the Indian subcontinent and is often called a "wonder tree". Considering the importance of neem in agriculture, medicine, industry, and environment, it is colloquially regarded as a tree for solving global problems [1]. India has a long history of using ethnomedicinal plants as traditional medicines (e.g., in Ayurveda, Unani, and Siddha). Neem is a preeminent natural resource containing a vast range of chemically diverse and structurally complex compounds possessing unique biological activities. It is interesting to note that over 400 secondary metabolites of different classes have already been reported from neem prospected from different ecological niches, which certainly justifies its historical use in the traditional medicinal sector ([1] and references therein).

Microbial associations are prevalent in plants; these multifaceted associations range from pathogenic, saprophytic, or opportunistic to more sustained mutualistic interactions such as mycorrhizal and endophytic (fungal, bacterial, and actinobacterial) [2]. Fungi are considered to be one of the most diverse life forms on earth. Although the magnitude of fungal diversity around the world is still open to debate, the estimate of hundreds to thousands of species to even millions has been put forth [2]. The most widely accepted estimate is that of Hawksworth [3] who estimated the size of 1.5 million species, out of which currently over 100000 species have been discovered. Among the different niches that support the growth of microorganisms, particularly fungi, one unique and specialized habitat is inter- and intracellular spaces of higher plants. These microorganisms are called endophytes. The term "endophyte" was first used by De Bary [4], and the commonly accepted definition pertains to "fungi or bacteria which for all or part of their life cycle, invade the tissues of living plant and cause unapparent and asymptomatic infections entirely within plant tissues, without disease" [5]. Mostert and co-workers (2000) further postulated that "true endophytes are fungi whose colonization never results in visible disease symptoms" [6]. It is now well established that endophytes are capable of maintaining mutualistic associations with their host plants, which often lead to the co-evolution of certain functional traits such as the production of bioactive secondary metabolites. During their co-existence with host plants, endophytes encounter invasion by a plethora of specific and generalist pathogens. Therefore, in order to survive in their ecological niches, endophytes might evolve additional defense strategies such as production of chemical defense compounds, small-molecule chemical modulators for activating host plant defenses, and precursors to host plant secondary metabolites, among others. Thus far, endophytes have emerged as relevant sources of biologically active natural products, and they play an essential role in maintaining the ecological balance in plants. Endophytes harboring neem are, therefore, also an invaluable resource of both novel as well as well-known natural compounds having high and diverse biological functionality for significant medicinal, agricultural, and industrial exploitation [7]. In this review, we provide a detailed elaboration on the metabolites identified to date in the neem plant as well as their biological activities. Further, we discuss thoroughly endophytes reported from neem 


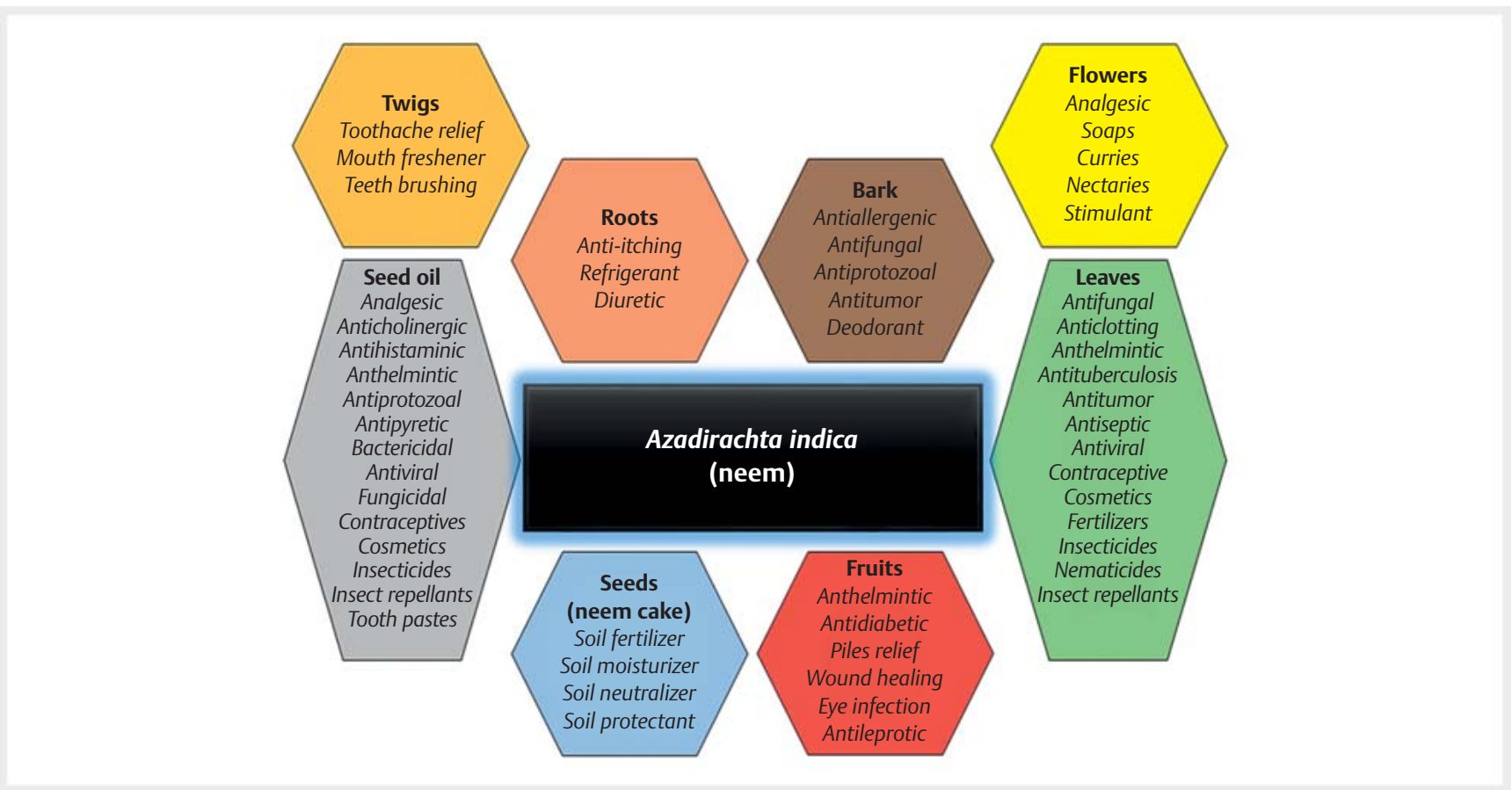

- Fig. 1 Important Azdirachta indica (neem) plant parts and their uses.

plants and the production of novel, biologically active metabolites.

\section{Neem, the "Wonder Tree"}

Neem (Azadirachta indica A. Juss.) is an indigenous medicinal plant of the Indian subcontinent [8]. The scientific name of this plant is derived from the Persian word "Azad dirakhat-I-Hind" meaning "noble or free tree of India" [9]. Neem had been described as early as 1830 by De Jussieu as an evergreen tree of the tropics and subtropics belonging to the family Meliaceae [10]. Medicinal properties of neem have been inscribed in the ancient testaments of Sanskrit literature as "Arishtha", which translates to "reliever of sickness". Dube (1996) described the ancient Indian names for the neem tree as prabhadra (very useful), paribhadrak (spreading its utility over vast distances), sarvobhadrak (useful in every way), and rajbhadrak (best among all the useful trees), all pointing towards its colossal worth in the Indian way of life [11]. Neem is widely used as a folk medicine for various therapeutic purposes as well as a source of agrochemicals for many centuries in the Indian agricultural system. The essential components of neem and their uses are summarized in > Fig. 1.

\section{Chemical Diversity of Compounds Reported from Neem}

Around 406 compounds have been isolated from different tissues of neem, and several sporadic reviews have also been published on the chemistry and structural diversity of these compounds. The compounds have been divided into 2 major classes: isopre- noids and non-isoprenoids ( $\bullet$ Fig. 2 ). On the one hand, isoprenoids include diterpenoids and triterpenoids encompassing protomeliacins, limonoids, azadirone, and its derivatives; gedunin and its derivatives; vilasinin type of compounds; and C-secomeliacins such as nimbin, salanin, and azadirachtin. On the other hand, non-isoprenoids are comprised of proteins (amino acids) and carbohydrates (polysaccharides), sulfur compounds, polyphenolics such as flavonoids and their glycosides, dihydrochalcone, coumarin, and tannins including aliphatic compounds, to name a few. The first isolated and characterized compound was nimbin, followed by nimbinin $[12,13]$. A considerable assortment of compounds have been isolated from different tissues of neem including leaves, twigs, flowers, fruits, seeds, seeds oil, bark, and roots, which are summarized in $>$ Table 1 along with their reported biological activities.

\section{Selected Bioactive Principles of Neem and Their Specific Activity}

\section{Anti-inflammatory, analgesic, and antipyretic activities}

Kaempferol, reported from neem as well as from a different, unrelated plant Rhamnus procumbens, was found to have anti-inflammatory and anti-ulcer activities [83,207]. Anti-inflammatory and immunomodulatory activity was observed in 2 flavonoids, catechin and epi-catechin, reported from the bark of the neem tree [177]. Nimbidin, a major active component of the Azadirachta indica seed oil, was found to significantly inhibit some of the functions of macrophages and neutrophils relevant to the inflamma- 


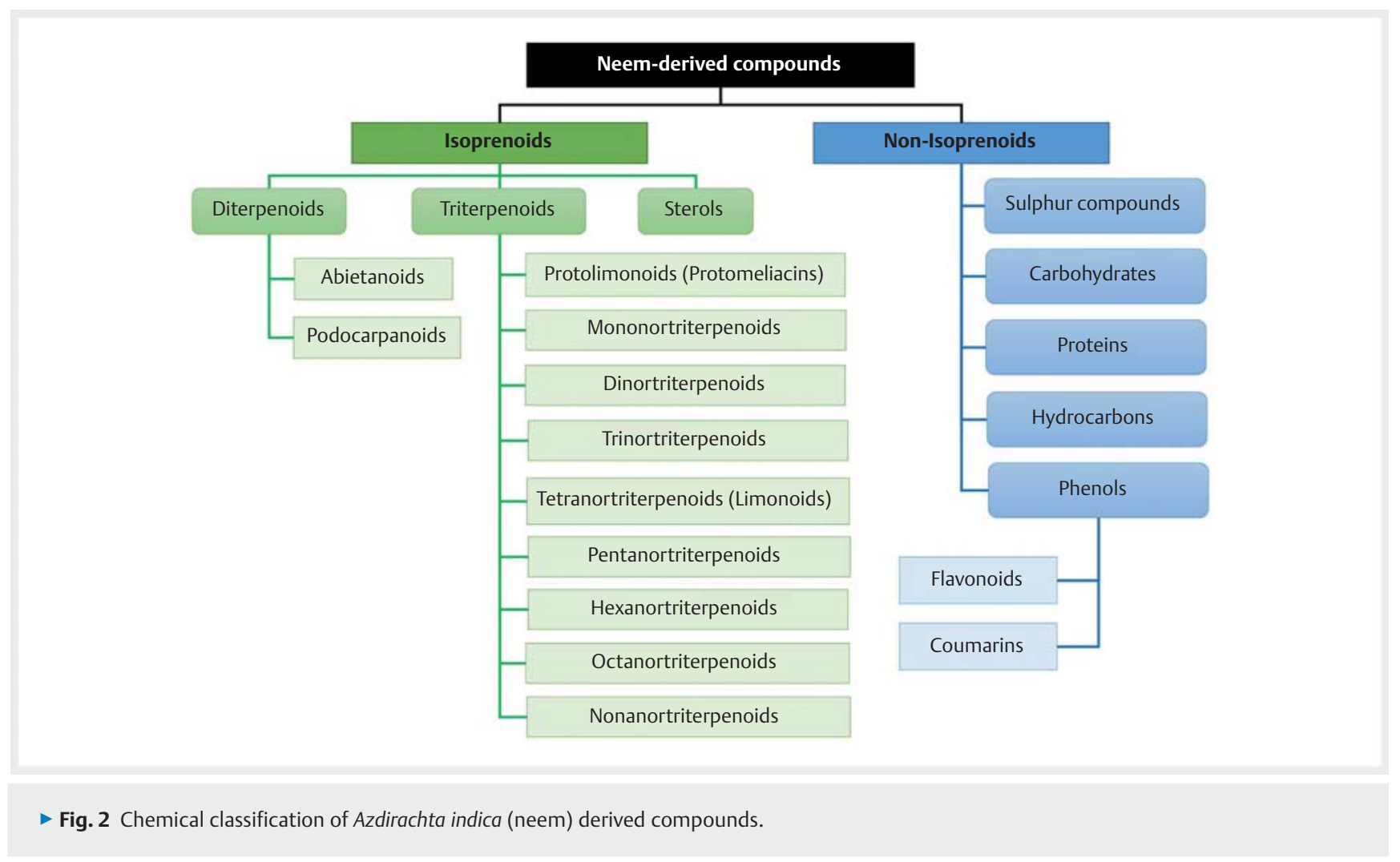

tory responses both in vitro as well as in vivo, signifying anti-inflammatory and anti-arthritic potential of the compound [209]. In another study, the effect of aqueous extract of neem leaves (400 mg/kg body weight) was compared with that of dexamethasone $(0.75 \mathrm{mg}$, intraperitoneal) in rats, which demonstrated statistically significant reduction by extract, albeit less pronounced compared to dexamethasone [210]. Crude ethanol extract of neem leaves yielded anti-inflammatory responses by suppression of paw edema induced by carrageenan and reduced cotton pelletinduced granuloma formation in chronic model rats [211]. Furthermore, the ethanol extract obtained from $A$. indica leaves $(1 \mathrm{~g} /$ $\mathrm{kg} \mathrm{w} / \mathrm{w}$ ) showed significant antinociceptive effect by inhibition of abdominal writhes produced by acetic acid. $A$. indica extract also showed statistically significant antipyretic effect $(p<0.05)$ at $1 \mathrm{~g} /$ $\mathrm{kg}$ and $500 \mathrm{mg} / \mathrm{kg}$ dose level on yeast-induced pyrexia in rats [211].

\section{Immunostimulant activities}

Neem oil is shown to selectively activate the cell-mediated immune mechanisms that elicit an enhanced response to subsequent mitogenic or antigenic challenges by acting as a non-specific immunostimulant [212]. Pre-treatment of rats with an odorous and volatile fraction of neem oil, coded NIM-76, was found to increase polymorphonuclear leukocytes, with a decrease in lymphocyte count displaying immunomodulatory efficacy [213].

\section{Radiosensitizing effects}

Neem oil was found to increase the radiosensitivity of the Balbc/ 3 T3 cells and severe combined immunodeficiency (SCID) cells during $x$-irradiation under aerobic conditions [214]. Application of neem oil reduced the $G 2+M$ phase of the cell cycle, thereby inhibiting the repair of cells from lethal damage [214].

\section{Hypoglycemic activities}

Neem kernel powder, in combination with glibenclamide, yielded significant antidiabetic and antihyperlipemic effects in alloxan diabetic rats [215]. Antihyperglycemic effect of aqueous neem leaf extract was also observed in insulin-dependent diabetes mellitus and non-insulin-dependent diabetes mellitus animal models [216]. Mixed water extracts of Abroma augusta roots, when combined with the leaves of $A$. indica and given orally to alloxan diabetic rats, showed hypoglycemic action with decreased formation of lipid peroxides estimated as thiobarbituric acid reactive substance along with increased antioxidants in erythrocytes [217]. Extracts of $A$. indica combined with extracts of Vernonia amygdalina (Del.) was found to have enhanced anti-diabetic effect in albino Wistar rats [218]. Ethanolic extracts of $A$. indica in streptozotocin-induced hyperglycemia normalized the glucose level and reversed dyslipidemia [219]. Hypoglycemic action of ethanolic neem leaf extract was evaluated in diabetic rats, which demonstrated that after treatment for $24 \mathrm{~h}$ with a single dose of $250 \mathrm{mg} / \mathrm{kg}$ extract reduced glucose (18\%), cholesterol (15\%), triglycerides (32\%), urea (13\%), creatinine (23\%), and lipids (15\%) [220]. Further, in a multiple-dose study that lasted for 15 days, reduction of creatinine, urea, lipids, triglycerides, and glucose were 
- Table 1 Biomolecules reported from different tissues of Azadirachta indica.

\begin{tabular}{|c|c|c|c|c|c|}
\hline SI. No. & Plant tissue & Compounds & Compound class & Biological activity & References \\
\hline 1 & Leaves & $\begin{array}{l}\text { Quercetin-3-o- } \beta \text {-d-gluco- } \\
\text { pyranoside (isoquercitrin) }\end{array}$ & Flavonoids & n.a. & [14] \\
\hline 2 & Leaves & $\begin{array}{l}\text { 3-hydroxystigmasta-5-en- } \\
\text { 7-one }\end{array}$ & Steroids & Antiplatelet aggregation & {$[15,16]$} \\
\hline 3 & Leaves & n-Hexacosanol & Miscellaneous compounds & $\begin{array}{l}\text { Potent feeding stimulants } \\
\text { for larvae of the silkworm }\end{array}$ & {$[17,18]$} \\
\hline 4 & Leaves & Tetratriacontane & Hydrocarbons & n.a. & [19] \\
\hline 5 & Leaves & Hexacosene & Hydrocarbons & n.a. & {$[19,20]$} \\
\hline 6 & Leaves & $\beta$-carotene & Hydrocarbons & n.a. & {$[21]$} \\
\hline 7 & Leaves & Nimbandiol & Pentanortriterpenoids & Antimalarial activity & {$[22]$} \\
\hline 8 & Leaves & $\alpha$-Linolenic acid & Fatty acids and their derivatives & Reduces cardiovascular disease & {$[23,24]$} \\
\hline 9 & Leaves & Xanthophylls & Miscellaneous compounds & Antioxidant activities & {$[20,21,25]$} \\
\hline 10 & Leaves & $\begin{array}{l}\text { Kaempferol-3-O-rutinoside } \\
\text { (or nicotiflorin) }\end{array}$ & Flavonoids & n.a. & [26] \\
\hline 11 & Leaves & Myricetin-3-O-rutinoside & Flavonoids & n.a. & {$[26]$} \\
\hline 12 & Leaves & $\begin{array}{l}\text { Quercetin-3-O- } \alpha \text {-L- } \\
\text { rhamnoside }\end{array}$ & Flavonoids & n.a. & [26] \\
\hline 13 & Leaves & Quercetin-3-O-rutinoside & Flavonoids & n.a. & {$[26]$} \\
\hline 14 & Leaves & Quercetrin & Flavonoids & $\begin{array}{l}\text { Inhibitors of azoxymethanol- } \\
\text { induced colonic neoplasia }\end{array}$ & {$[27-31]$} \\
\hline 15 & Leaves & Rutin & Flavonoids & $\begin{array}{l}\text { Inhibitors of azoxymethanol- } \\
\text { induced colonic neoplasia }\end{array}$ & [27-29] \\
\hline 16 & Leaves & Oxalic acid & Acids and their derivatives & n.a. & [32] \\
\hline 17 & Leaves & Ascorbic acid & Acids and their derivatives & n.a. & [32] \\
\hline 18 & Leaves & Melianol & Mononotriterpenoids & n.a. & {$[32]$} \\
\hline 19 & Leaves & 2',3'-dehydrosalannol & $\begin{array}{l}\text { Ring-C-seco-tetrano- } \\
\text { triterpenoids }\end{array}$ & n.a. & [33] \\
\hline 20 & Leaves & $\begin{array}{l}\text { 1,3-diacetyl-11,19-deoxo- } \\
\text { 19-oxomelia-carpin }\end{array}$ & $\begin{array}{l}\text { Ring-C-seco-tetrano- } \\
\text { triterpenoids }\end{array}$ & n.a. & [34] \\
\hline 21 & Leaves & Isoazadirolide & $\begin{array}{l}\text { Ring-C-seco-tetrano- } \\
\text { triterpenoids }\end{array}$ & n.a. & [35] \\
\hline 22 & Leaves & $\begin{array}{l}\text { Desfurano-6- } \alpha \text {-hydroxy- } \\
\text { azadiradione }\end{array}$ & Octanortriterpenoids & $\begin{array}{l}\text { Insecticidal activity against } \\
\text { fourth instar larvae of } \\
\text { mosquito (Anopheles stephensi) }\end{array}$ & [36] \\
\hline 23 & Leaves & 22, 23-dihydronimocinol & Tetranortriterpenoids & $\begin{array}{l}\text { Insecticidal activity fourth } \\
\text { instar larvae of the mosquito } \\
\text { (Anopheles stephensi) }\end{array}$ & [36] \\
\hline 24 & Leaves & Nimbocinolide & $\gamma$-Hydroxybutenolides & n.a. & [37] \\
\hline 25 & Leaves & Isonimbocinolide & $\gamma$-Hydroxybutenolides & n.a. & {$[38]$} \\
\hline 26 & Leaves & 23-O-methylnimocinolide & $y$-Hydroxybutenolides & $\begin{array}{l}\text { Insect growth regulating } \\
\text { effect on mosquitoes } \\
\text { (Aedes aegypti) }\end{array}$ & [39] \\
\hline 27 & Leaves & $\begin{array}{l}\text { 1,7-O-deacetyl-23-O-methyl- } \\
7 \alpha \text {-O-senecioylnimocinolide }\end{array}$ & $\gamma$-Hydroxybutenolides & $\begin{array}{l}\text { Insect growth regulating } \\
\text { effect on mosquitoes } \\
\text { (Aedes aegypti) }\end{array}$ & [39] \\
\hline 28 & Leaves & Meldenin & Tetranortriterpenoids & Antimalarial activity & [40-42] \\
\hline 29 & Leaves & Isomeldenin & Tetranortriterpenoids & Antimalarial activity & [41-43] \\
\hline 30 & Leaves & Meldenindiol & Tetranortriterpenoids & n.a. & {$[41,43]$} \\
\hline 31 & Leaves & $\beta$-sitosterol & Steroids & Insecticidal activity & {$[20,44]$} \\
\hline 32 & Leaves & Zafaral & Tetranortriterpenoids & $\begin{array}{l}\text { Insect growth regulating } \\
\text { against houseflies and toxic } \\
\text { on larvae of mosquitoes }\end{array}$ & {$[45]$} \\
\hline
\end{tabular}


- Table 1 Continued

\begin{tabular}{|c|c|c|c|c|c|}
\hline SI. No. & Plant tissue & Compounds & Compound class & Biological activity & References \\
\hline 33 & Fresh leaves & Zeeshanol & Trinortriterpenoids & n.a. & [46] \\
\hline 34 & Dried leaves & $\begin{array}{l}4 \alpha, 6 \alpha \text {-dihydroxy-A- } \\
\text { homozadirone }\end{array}$ & Tetranortriterpenoids & n.a. & [47] \\
\hline 35 & Air dried leaves & Azadirachtolide & Tetranortriterpenoids & n.a. & [48] \\
\hline 36 & Air dried leaves & Deoxyazadirachtolide & Tetranortriterpenoids & n.a. & [48] \\
\hline 37 & Air dried leaves & $\begin{array}{l}\text { Sulfonoquinovosyldiacyl- } \\
\text { glyceride }\end{array}$ & Glyceride & Antileukemic activity & [49] \\
\hline 38 & Fresh leaves & Azadirachtanin & Tetranortriterpenoids & Hyperglycemic activity & {$[50,51]$} \\
\hline 39 & Fresh leaves & Nimocinolide & $\gamma$-Hydroxybutenolides & $\begin{array}{l}\text { Insect growth-regulating } \\
\text { properties }\end{array}$ & [44] \\
\hline 40 & Fresh leaves & Isonimocinolide & $\gamma$-Hydroxybutenolides & $\begin{array}{l}\text { Insect growth- regulating } \\
\text { properties }\end{array}$ & [44] \\
\hline 41 & Undried leaves & Nimbocinone & Protolimonoids & n.a. & [51] \\
\hline 42 & Leaves extract & Meliacinanhydride & Tetranortriterpenoids & n.a. & [45] \\
\hline 43 & $\begin{array}{l}\text { Undried winter } \\
\text { leaves }\end{array}$ & $\begin{array}{l}\text { Nimocinol (nimonol or } \\
\text { 6-hydroxyazadirone) }\end{array}$ & Tetranortriterpenoids & Antimalarial activity & {$[42,52]$} \\
\hline 44 & $\begin{array}{l}\text { Fresh green } \\
\text { whole leaves }\end{array}$ & 14, 15-epoxynimonol & Tetranortriterpenoids & n.a. & [53] \\
\hline 45 & Fresh leaves & $\begin{array}{l}\text { Desfurano-desacetylnimbin- } \\
\text { 17-one }\end{array}$ & $\begin{array}{l}\text { Octanortriterpenoids (ring-C } \\
\text { seco-octanortriterpenoids) }\end{array}$ & n.a. & [54] \\
\hline 46 & $\begin{array}{l}\text { Chloroform } \\
\text { extract of dried } \\
\text { leaves }\end{array}$ & Meliacinolin & Tetranortriterpenoid & Antidiabetic & [55] \\
\hline 47 & $\begin{array}{l}\text { Methanolic } \\
\text { extract of fresh } \\
\text { leaves }\end{array}$ & Odoratone & Protolimonoids & $\begin{array}{l}\text { Mortality on fourth instar } \\
\text { larvae of mosquitoes } \\
\text { (Anopheles stephensi) }\end{array}$ & [56] \\
\hline 48 & $\begin{array}{l}\text { Methanolic } \\
\text { extract of fresh } \\
\text { leaves }\end{array}$ & Meliatetraolenone & Tetranortriterpenoids & Insecticidal activity & [56] \\
\hline 49 & $\begin{array}{l}\text { Methanolic } \\
\text { extract of the } \\
\text { fresh leaves }\end{array}$ & $\begin{array}{l}6 \alpha \text {-O-acetyl-7-deacetyl- } \\
\text { nimocinol }\end{array}$ & Tetranortriterpenoids & $\begin{array}{l}\text { Toxicity on fourth instar larvae } \\
\text { of mosquitoes (Aedes aegypti) }\end{array}$ & [57] \\
\hline 50 & $\begin{array}{l}\text { Methanolic } \\
\text { extract of the } \\
\text { fresh leaves }\end{array}$ & Meliacinol & Tetranortriterpenoids & $\begin{array}{l}\text { Protection against Tacaribe } \\
\text { virus to mice brain }\end{array}$ & [57] \\
\hline 51 & $\begin{array}{l}\text { Powdered } \\
\text { neem leaves }\end{array}$ & $\begin{array}{l}\text { 3-deacetyl-3-cinnamoyl } \\
\text { azadirachtin }\end{array}$ & $\begin{array}{l}\text { Ring-C-seco-tetrano- } \\
\text { triterpenoids }\end{array}$ & n.a. & [58] \\
\hline 52 & Flower & Neeflone & Tetranortriterpenoid & n.a. & [59] \\
\hline 53 & Flower & Flowerone & Flavonoids & n.a. & [60] \\
\hline 54 & Flower & Flowerin & Flavonoids & n.a. & {$[60]$} \\
\hline 55 & Flower & Azharone & Flavonoids & n.a. & [61] \\
\hline 56 & Flower & $3^{\prime}$-prenylnaringenin & Flavonoids & n.a. & [60] \\
\hline 57 & Flower & Trichilenone acetate & Triterpenoid & n.a. & {$[60]$} \\
\hline 58 & Flower & $\begin{array}{l}\text { Prenylated flavanones, 5,7,4'- } \\
\text { trihydroxy-8-prenylflavanone }\end{array}$ & Flavanones & Antimutagenic & [62] \\
\hline 59 & Flower & $\begin{array}{l}\text { 5,4'-dihydroxy-7-methoxy- } \\
\text { 8-prenylflavanone }\end{array}$ & Flavanones & Antimutagenic & {$[62]$} \\
\hline 60 & Flower & $\begin{array}{l}\text { 5,7,4'-trihydroxy-3',8- } \\
\text { diprenylflavanone }\end{array}$ & Flavanones & Antimutagenic & {$[62]$} \\
\hline 61 & Flower & $\begin{array}{l}\text { 5,7,4'-trihydroxy-3',5'- } \\
\text { diprenylflavanone }\end{array}$ & Flavanones & Antimutagenic & [62] \\
\hline 62 & Flowers & $\begin{array}{l}\text { Melicitrin (a glycoside } \\
\text { of myricetin) }\end{array}$ & Flavonoids & n.a. & [63] \\
\hline
\end{tabular}


- Table 1 Continued

\begin{tabular}{|c|c|c|c|c|c|}
\hline SI. No. & Plant tissue & Compounds & Compound class & Biological activity & References \\
\hline 63 & Flowers & $\begin{array}{l}\text { Kaempferol-3-glucoside (also } \\
\text { called astragalin) }\end{array}$ & Flavonoids & n.a. & [63] \\
\hline 64 & Flowers & $\begin{array}{l}\text { Quercetin-3-galactoside (or } \\
\text { hyperin) }\end{array}$ & Flavonoids & n.a. & [63] \\
\hline 65 & Flowers & Myricetin-3-arabinoside & Flavonoids & n.a. & {$[63]$} \\
\hline 66 & Fresh flowers & Diepoxyazadirol & Apo-protolimonoids & n.a. & {$[60]$} \\
\hline 67 & Fresh flowers & Trichilinone & Tetranortriterpenoids & n.a. & {$[60]$} \\
\hline 68 & Fresh flowers & O-methylazadironolide & $\gamma$-Hydroxybutenolides & n.a. & {$[60]$} \\
\hline 69 & Fresh flowers & $\begin{array}{l}\text { 1S,2S,5R-1,4,4-Trimethyl- } \\
\text { tricyclo[6.3.1.0 }(2,5)] \text { dodec- } \\
\text { 8-ene }\end{array}$ & Sesquiterpenoids & n.a. & {$[64]$} \\
\hline 70 & Fresh flowers & $\begin{array}{l}\text { 2-Methoxy-5,4'-dimethyl- } \\
\text { benzenebutanal }\end{array}$ & n.a. & n.a. & {$[64]$} \\
\hline 71 & Fresh flowers & $\begin{array}{l}\text { 1,4-Dimethoxy-2-(methyl- } \\
\text { thio)-benzene }\end{array}$ & n.a. & n.a. & {$[64]$} \\
\hline 72 & Fresh flowers & $\begin{array}{l}\text { n-Hexadecanoic acid } \\
\text { or palmitic acid }\end{array}$ & n.a. & n.a. & [64] \\
\hline 73 & Fresh flowers & $\begin{array}{l}\text { Methyl n-hexadecanoate } \\
\text { or methyl palmitate }\end{array}$ & n.a. & n.a. & {$[64]$} \\
\hline 74 & Fresh flowers & $\begin{array}{l}\text { Methyl octadecanoate acid } \\
\text { or methyl stearate }\end{array}$ & n.a. & n.a. & {$[64]$} \\
\hline 75 & Fresh flowers & $\begin{array}{l}\text { Methyl 17-hydroxymethyl- } \\
\text { octadecanoate }\end{array}$ & n.a. & n.a. & {$[64]$} \\
\hline 76 & Fresh flowers & $\begin{array}{l}\text { Decahydro-1,1,3a-trimethyl- } \\
\text { 7-methylene-[1aS-(1aa', 3aa', } \\
\text { 7aa', 7ba']-1H-cyclopropa[a] } \\
\text { naphthalene }\end{array}$ & Sesquiterpenoids & n.a. & [64] \\
\hline 77 & Fresh flowers & $\begin{array}{l}\text { 1,1,4,8-Tetramethyl-cis,cis,cis- } \\
4,7,10 \text {-cycloundecatriene }\end{array}$ & Sesquiterpenoids & n.a. & [64] \\
\hline 78 & Fresh flowers & $\begin{array}{l}\text { Methyl docosanoate } \\
\text { or methyl beheniate }\end{array}$ & n.a. & n.a. & [64] \\
\hline 79 & Fresh flowers & $\begin{array}{l}\text { Methyl dodecanoate } \\
\text { or methyl laurate }\end{array}$ & n.a. & n.a. & {$[64]$} \\
\hline 80 & Fresh flowers & $\begin{array}{l}\text { Methyl tetradecanoate } \\
\text { or methyl myristate }\end{array}$ & n.a. & n.a. & {$[64]$} \\
\hline 81 & Fresh flowers & Methyl eicosanoate & Hydrocarbons & n.a. & {$[64]$} \\
\hline 82 & Fresh flowers & Methyl heptadecanoate & Hydrocarbons & n.a. & {$[64]$} \\
\hline 83 & Fresh flowers & Methyl hexacosanoate & Hydrocarbons & n.a. & {$[64]$} \\
\hline 84 & Fresh flowers & $\begin{array}{l}\text { Methyl 15-methylhepta- } \\
\text { decanoate }\end{array}$ & Hydrocarbons & n.a. & {$[64]$} \\
\hline 85 & Fresh flowers & $\begin{array}{l}\text { Methyl 14-methylpenta- } \\
\text { decanoate }\end{array}$ & Hydrocarbons & n.a. & {$[64]$} \\
\hline 86 & Fresh flowers & Methyl 12-methyltridecanoate & Hydrocarbons & n.a. & {$[64]$} \\
\hline 87 & Fresh flowers & $\begin{array}{l}\text { Nonanedioic acid } \\
\text { monomethylester }\end{array}$ & Hydrocarbons & n.a. & {$[64]$} \\
\hline 88 & Fresh flowers & Methyl octacosanoate & Hydrocarbons & n.a. & {$[64]$} \\
\hline 89 & Fresh flowers & Methyl 9-oxononaoate & Hydrocarbons & n.a. & {$[64]$} \\
\hline 90 & Fresh flowers & Methyl 4-oxooctanoate & Hydrocarbons & n.a. & {$[64]$} \\
\hline 91 & Fresh flowers & Methyl pentacosanoate & Hydrocarbons & n.a. & {$[64]$} \\
\hline 92 & Fresh flowers & Methyl tetracosanoate & Hydrocarbons & n.a. & [64] \\
\hline 93 & Fresh flowers & $\begin{array}{l}\text { Methyl 9-methyltetra- } \\
\text { decanoate }\end{array}$ & Hydrocarbons & n.a. & {$[64]$} \\
\hline
\end{tabular}


- Table 1 Continued

\begin{tabular}{|c|c|c|c|c|c|}
\hline SI. No. & Plant tissue & Compounds & Compound class & Biological activity & References \\
\hline 94 & Fresh flowers & Methyl 8-(2-furyl)-octanoate & Hydrocarbons & n.a. & {$[64]$} \\
\hline 95 & Fresh flowers & Germacrene B & Sesquiterpenoids & n.a. & {$[64]$} \\
\hline 96 & Fresh flowers & a-himachalene & Sesquiterpenoids & n.a. & [64] \\
\hline 97 & Fresh flowers & $\alpha$-Sitosterol & Steroids & n.a. & [64] \\
\hline 98 & Fresh flowers & $\alpha$-Sitosterol acetate & Steroids & n.a. & {$[64]$} \\
\hline 99 & Fresh flowers & Lanosterol & Steroids & n.a. & {$[64]$} \\
\hline 100 & Fresh flowers & Tyrosol & Phenolic constituents & n.a. & {$[60]$} \\
\hline 101 & Fresh flowers & Dodecasanoic acid & Fatty acids and their derivatives & n.a. & [15] \\
\hline 102 & Fresh flowers & $\begin{array}{l}\text { Heptadecanoic acid } \\
\text { or margarnoic acid }\end{array}$ & Fatty acids and their derivatives & n.a. & [15] \\
\hline 103 & Fresh flowers & $\begin{array}{l}\text { Hexacosanoic acid } \\
\text { or cerotic acid }\end{array}$ & Fatty acids and their derivatives & n.a. & [15] \\
\hline 104 & Fresh flowers & 15-methylheptadecanoic acid & Fatty acids and their derivatives & n.a. & [15] \\
\hline 105 & Fresh flowers & $\begin{array}{l}\text { 12-methyltridecanoic acid } \\
\text { or isomyristic acid }\end{array}$ & Fatty acids and their derivatives & n.a. & [15] \\
\hline 106 & Fresh flowers & Nonanedioic acid & Fatty acids and their derivatives & n.a. & [64] \\
\hline 107 & Fresh flowers & Octacosanoic acid & Fatty acids and their derivatives & n.a. & [15] \\
\hline 108 & Fresh flowers & $\begin{array}{l}\text { Octadecanoic acid } \\
\text { or stearic acid }\end{array}$ & Fatty acids and their derivatives & n.a. & {$[64]$} \\
\hline 109 & Fresh flowers & $\begin{array}{l}\text { 9-oxononaoic acid } \\
\text { or azelaldehydic acid }\end{array}$ & Fatty acids and their derivatives & n.a. & [15] \\
\hline 110 & Fresh flowers & 4-oxooctanoic acid & Fatty acids and their derivatives & n.a. & [15] \\
\hline 111 & Fresh flowers & Pentacosanoic acid & Fatty acids and their derivatives & n.a. & {$[15]$} \\
\hline \multirow[t]{2}{*}{112} & Fresh flowers & 9-methyltetradecanoic acid & Fatty acids and their derivatives & n.a. & [15] \\
\hline & Fresh flowers & 17-hydroxysteric acid & Fatty acids and their derivatives & n. a. & [15] \\
\hline 113 & Fresh fruits & Melianone & Protolimonoids & Antifeeding activity & {$[65,66]$} \\
\hline 114 & Fresh fruits & Nimolinone & Protolimonoids & n.a. & {$[65,67]$} \\
\hline 115 & Fresh fruits & Nimocin & Tetranortriterpenoids & n.a. & {$[44]$} \\
\hline 116 & Fresh fruits & Nimbocinol & Tetranortriterpenoids & $\begin{array}{l}\text { Insect growth inhibitory } \\
\text { activity }\end{array}$ & {$[44,67]$} \\
\hline 117 & Fresh ripe fruits & Azadirachtol & Apo-protolimonoids & Potent antifeedant & [68-70] \\
\hline 118 & Fresh ripe fruits & Azadirachnol (naheedin) & Apo-protolimonoids & n.a. & [71] \\
\hline 119 & Fresh ripe fruits & Azadirol & Apo-protolimonoids & п.а. & {$[72]$} \\
\hline 120 & Fruits & 17-epi-Azadiradione & Tetranortriterpenoids & n.a. & [73] \\
\hline 121 & Fruits & $17-\beta$-hydroxyazadiradione & Tetranortriterpenoids & $\begin{array}{l}\text { Antifeedant activities and } \\
\text { growth inhibitor activity } \\
\text { against larvae of rice leaf folder }\end{array}$ & {$[73,74]$} \\
\hline 122 & Fruits & Nimbocetin & Acids and their derivatives & n.a. & [31] \\
\hline 123 & Fruits & 5-hydroxymethylfurfural & Miscellaneous compounds & n.a. & [31] \\
\hline 124 & Fruits & Ochinolide B & $\begin{array}{l}\text { Ring-C-seco-tetrano- } \\
\text { triterpenoids }\end{array}$ & n. a. & {$[75,76]$} \\
\hline 125 & Fruits & Nimbochalcin & Coumarin and chalcones & n.a. & [31] \\
\hline 126 & Fresh fruit coats & Azadironic acid & Tetranortriterpenoids & $\begin{array}{l}\text { Toxic against mosquito } \\
\text { (Anopheles stephensi) }\end{array}$ & {$[77,78]$} \\
\hline 127 & Fresh fruit coats & Limocinin & Tetranortriterpenoids & n.a. & [79] \\
\hline 128 & Fresh fruit coats & Limocin-A & Tetranortriterpenoids & Insecticidal activity & {$[77,79]$} \\
\hline 129 & Fresh fruit coats & Limocin-B & Tetranortriterpenoids & Insecticidal activity & {$[77,79]$} \\
\hline 130 & Fresh fruit coats & Limocin-C & Tetranortriterpenoids & n.a. & [80] \\
\hline 131 & Fresh fruit coats & Limocin-D & Tetranortriterpenoids & n. a. & {$[80]$} \\
\hline
\end{tabular}


- Table 1 Continued

\begin{tabular}{|c|c|c|c|c|c|}
\hline SI. No. & Plant tissue & Compounds & Compound class & Biological activity & References \\
\hline 132 & Fresh fruit coats & Meliacinolactol & Trinortriterpene & n.a. & {$[80]$} \\
\hline 133 & $\begin{array}{l}\text { Fresh undried } \\
\text { ripe fruits }\end{array}$ & Nimolicinolic acid & Hexanortriterpenoids & n.a. & {$[81]$} \\
\hline 134 & Fresh fruit coats & Desfuranoazadiradione & Octanortriterpenoids & $\begin{array}{l}\text { Insect growth regulating } \\
\text { activity }\end{array}$ & {$[77,82]$} \\
\hline 135 & $\begin{array}{l}\text { Fresh fruit } \\
\text { coatings }\end{array}$ & $\begin{array}{l}\text { Galoxolide or 1,3,4,6,7,8-hexa- } \\
\text { hydro-4,6,6,7,8,8-hexamethyl- } \\
\text { cyclopenta[g]-2-benzopyran }\end{array}$ & n.a. & n.a. & {$[17,83]$} \\
\hline 136 & $\begin{array}{l}\text { Fresh fruit } \\
\text { coatings }\end{array}$ & $\begin{array}{l}\text { Methyl 14-methyl-penta- } \\
\text { decanoate }\end{array}$ & n.a. & n.a. & {$[83]$} \\
\hline 137 & $\begin{array}{l}\text { Fresh fruit } \\
\text { coatings }\end{array}$ & $\begin{array}{l}\text { 2,6-bis(1,1-dimethylethyl)- } \\
\text { 4-methyl phenol }\end{array}$ & n.a. & n.a. & {$[83]$} \\
\hline 138 & $\begin{array}{l}\text { Fresh fruit } \\
\text { coatings }\end{array}$ & $\begin{array}{l}\text { 2-(phenylmethylene)-octanal } \\
\text { or } \alpha \text {-hexylcinnamaldehyde }\end{array}$ & n.a. & n.a. & {$[83]$} \\
\hline 139 & $\begin{array}{l}\text { Fresh fruit } \\
\text { coatings }\end{array}$ & $\begin{array}{l}\text { Ethyl hexadecanoate } \\
\text { or ethyl palmitate }\end{array}$ & n.a. & n.a. & {$[83]$} \\
\hline 140 & $\begin{array}{l}\text { Fresh fruit } \\
\text { coatings }\end{array}$ & $\begin{array}{l}\text { Ethyl 9Z-octadecenoate } \\
\text { or ethyl oleate }\end{array}$ & n.a. & n.a. & {$[83]$} \\
\hline 141 & $\begin{array}{l}\text { Fresh fruit } \\
\text { coatings }\end{array}$ & $\begin{array}{l}\text { Methyl 14-methyl } \\
\text { pentadecanoate }\end{array}$ & n.a. & n.a. & {$[83]$} \\
\hline 142 & $\begin{array}{l}\text { Fresh fruit } \\
\text { coatings }\end{array}$ & $\begin{array}{l}\text { 3,7-dimethyl-1-octen-7-ol } \\
\text { or dihydromyrcenol }\end{array}$ & n.a. & n.a. & {$[83]$} \\
\hline 143 & Fresh fruit & Azadironol & Octanortriterpenoids & n.a. & {$[77,84]$} \\
\hline 144 & Fruit coat extract & Meliacinin & Dinortriterpenoids & $\begin{array}{l}\text { Toxic against mosquito } \\
\text { (Anopheles stephensi) }\end{array}$ & {$[77,78]$} \\
\hline 145 & Fresh fruit coats & Salimuzzalin & Tetranortriterpenoids & n.a. & {$[40,84]$} \\
\hline 146 & Fruit coatings & Limocinol & Protolimonoids & n.a. & {$[79]$} \\
\hline 147 & Fruit coatings & Limocinone & Protolimonoids & n.a. & {$[79]$} \\
\hline 148 & Fruit coatings & Kulactone & Protolimonoids & n.a. & {$[72]$} \\
\hline 149 & Fruit coats & $\begin{array}{l}\text { Methyl (2E, 6E)-farnesoate } \\
\text { or Methyl-3,7,11-trimethyl- } \\
\text { 2E,6E,10-dodecatrienoate }\end{array}$ & Sesquiterpenoids & n.a. & {$[17,83]$} \\
\hline 150 & Fruit coats & Dihydromyreenol & Monoterpenoids & n.a. & {$[17]$} \\
\hline 151 & Fruit coats & T-butylated hydroxytoluene & Phenolic constituents & n.a. & {$[17]$} \\
\hline 152 & Fruit coats & $\alpha$-hexyl cinnamaldehyde & Phenolic constituents & n.a. & {$[17]$} \\
\hline 153 & Fruit coats & $\beta$-asarone & Phenolic constituents & Antifungal activity & {$[17,83,85]$} \\
\hline 154 & Fruit coats & Azadirolic acid & Dinortriterpenoids & n.a. & {$[84]$} \\
\hline 155 & Fruit coats & Azadiradionol & Dinortriterpenoids & n.a. & {$[84]$} \\
\hline 156 & Fruit coats & Azadironolide & $\gamma$-Hydroxybutenolides & n.a. & {$[86]$} \\
\hline 157 & Fruit coats & Isoazadirodionolide & $\gamma$-Hydroxybutenolides & n.a. & {$[86]$} \\
\hline 158 & Fruit coats & Azadiradionolide & $\gamma$-Hydroxybutenolides & n.a. & {$[86]$} \\
\hline 159 & Fruit coats & Icosane & Hydrocarbons & n.a. & {$[71]$} \\
\hline 160 & Fruit coats & Docosane & Hydrocarbons & n.a. & {$[71]$} \\
\hline 161 & Fruit coats & 2-methyltricosane & Hydrocarbons & n.a. & [71] \\
\hline 162 & Fruit coats & Docosene & Hydrocarbons & n.a. & [71] \\
\hline 163 & Fruit coats & n-pentadecane & Hydrocarbons & n.a. & [83] \\
\hline 164 & Fruit coats & n-hexadecane & Hydrocarbons & n.a. & [83] \\
\hline 165 & Fruit coats & n-heptadecane & Hydrocarbons & n.a. & [83] \\
\hline 166 & Fruit coats & n-tetracosane & Hydrocarbons & n.a. & [83] \\
\hline 167 & Fruit coats & n-hexacosane & Hydrocarbons & n.a. & [83] con \\
\hline
\end{tabular}


- Table 1 Continued

\begin{tabular}{|c|c|c|c|c|c|}
\hline SI. No. & Plant tissue & Compounds & Compound class & Biological activity & References \\
\hline 168 & $\begin{array}{l}\text { Fresh, undried, } \\
\text { and unruptured } \\
\text { ripe fruits }\end{array}$ & Isonimolicinolide & Tetranortriterpenoids & n.a. & [81] \\
\hline 169 & Seeds & Vepaol & $\begin{array}{l}\text { Ring-C-seco-tetrano- } \\
\text { triterpenoids }\end{array}$ & n.a. & {$[58,87,88]$} \\
\hline 170 & Seeds & Isovepaol & $\begin{array}{l}\text { Ring-C-seco-tetrano- } \\
\text { triterpenoids }\end{array}$ & n.a. & {$[88,89]$} \\
\hline 171 & Seeds & Indole acetic acid & Acids and their derivatives & Plant growth hormone & [90] \\
\hline 172 & Seeds & Indole pyruvic acid & Acids and their derivatives & n.a. & [90] \\
\hline 173 & Seeds & Tiglic acid & Acids and their derivatives & n.a. & [90] \\
\hline 174 & Seeds & 7-deacetylazadiradione & Tetranortriterpenoids & n.a. & [91] \\
\hline 175 & Seeds & Epoxyazadiradione & Tetranortriterpenoids & $\begin{array}{l}\text { Insecticidal activity, anti- } \\
\text { inflammatory activity }\end{array}$ & {$[71,91,92]$} \\
\hline 176 & Seeds & 7-acetylneotrichilenone & Tetranortriterpenoids & n.a. & [91] \\
\hline 177 & Seeds & $\begin{array}{l}\text { 7-benzoyl-17-hydroxy- } \\
\text { nimbocinol }\end{array}$ & Tetranortriterpenoids & n.a. & [93] \\
\hline 178 & Seeds & 15-hydroxyazadiradione & Tetranortriterpenoids & n.a. & [93] \\
\hline 179 & Seeds & 23-deoxyazadironolide & $\gamma$-Hydroxybutenolides & n.a. & [93] \\
\hline 180 & Seeds & Limocin $E$ & Tetranortriterpenoids & n.a. & [93] \\
\hline 181 & Seeds & 23-epilimocin E & Tetranortriterpenoids & n.a. & [93] \\
\hline 182 & Seeds & $\begin{array}{l}\text { 7a-acetoxy-3-oxoisocopala- } \\
\text { 1,13-dien-15-oic acid }\end{array}$ & Diterpenoid & n.a. & [93] \\
\hline 183 & Dried seeds & $\begin{array}{l}1 \alpha \text {-methoxy-1, 2-dihydro- } \\
\text { nimbinin }\end{array}$ & Tetranortriterpenoids & n.a. & [91] \\
\hline 184 & Dried seeds & $1 \beta, 2 \beta$-diepoxyazadiradione & Tetranortriterpenoids & n.a. & [91] \\
\hline 185 & Seed oil & $\begin{array}{l}1 \alpha, 2 \alpha \text {-epoxy- } 17 \beta \text {-hydroxy- } \\
\text { azadiradione }\end{array}$ & Tetranortriterpenoids & n.a. & [94] \\
\hline 186 & Seed oil & Vepinin & Tetranortriterpenoids & n.a. & [95] \\
\hline 187 & Seed oil & Mahmoodin & Tetranortriterpenoids & $\begin{array}{l}\text { Effective against human } \\
\text { pathogenic bacteria }\end{array}$ & {$[71,96]$} \\
\hline 188 & Seed oil & $1 \alpha, 2 \alpha$-epoxynimolicinol & Tetranortriterpenoids & n.a. & [94] \\
\hline 189 & Seed oil & 7-deacetylnimolicinol & Tetranortriterpenoids & n.a. & [94] \\
\hline 190 & Seed oil & Vilasinin triacetate & Tetranortriterpenoids & n.a. & [43] \\
\hline 191 & Seed oil & $\begin{array}{l}\text { 1-O-senecioyl-3-O- } \\
\text { acetylvilasinin }\end{array}$ & Tetranortriterpenoids & n.a. & [97] \\
\hline 192 & Seed Oil & Di-, tri- and tetra- sulfides & $\begin{array}{l}\text { Sulphur containing } \\
\text { compounds }\end{array}$ & n.a. & {$[98,99]$} \\
\hline 193 & Seed oil & Gedunin & $\begin{array}{l}\text { Tetranortriterpenoids } \\
\text { (meliacins/limonoids) }\end{array}$ & $\begin{array}{l}\text { Antifeedant activities and } \\
\text { growth inhibitor activity } \\
\text { against larvae of rice leaffolder, } \\
\text { In vitro cytotoxic activities }\end{array}$ & $\begin{array}{l}{[71,74,100} \\
101]\end{array}$ \\
\hline 194 & Seed oil & 7-decacetylgedunin & Tetranortriterpenoids & $\begin{array}{l}\text { Antifeedant activities and } \\
\text { growth inhibitor activity } \\
\text { against larvae of rice leaffolder }\end{array}$ & {$[74,100]$} \\
\hline 195 & Seed Oil & Nimolicinol & Tetranortriterpenoids & $\begin{array}{l}\text { Antibacterial to several human } \\
\text { pathogenic bacteria }\end{array}$ & {$[52,94,96]$} \\
\hline 196 & Seed oil & 17-epi-Nimbocinol & Tetranortriterpenoids & n.a. & [102] \\
\hline 197 & Neem oil & $\begin{array}{l}\text { Methyl esters of } 14 \text {-methyl- } \\
\text { pentadecanoic acid }\end{array}$ & Fatty acids and their derivatives & n.a. & [17] \\
\hline 198 & Neem oil & Methyl esters of ethyl palmate & Fatty acids and their derivatives & n.a. & [17] \\
\hline 199 & Neem oil & Methyl esters of oleate & Fatty acids and their derivatives & n.a. & [17] \\
\hline
\end{tabular}


- Table 1 Continued

\begin{tabular}{|c|c|c|c|c|c|}
\hline SI. No. & Plant tissue & Compounds & Compound class & Biological activity & References \\
\hline 200 & Neem oil & Salannin & $\begin{array}{l}\text { Ring-C-seco-tetrano- } \\
\text { triterpenoids }\end{array}$ & Antifeeding properties & {$[76,103-106]$} \\
\hline 201 & Neem oil & 3-deacetylsalannin & $\begin{array}{l}\text { Ring-C-seco-tetrano- } \\
\text { triterpenoids }\end{array}$ & Antifeeding properties & {$[22,104,107]$} \\
\hline 202 & Neem oil & Salannol & $\begin{array}{l}\text { Ring-C-seco-tetrano- } \\
\text { triterpenoids }\end{array}$ & Antifeeding properties & {$[22]$} \\
\hline 203 & Neem oil & Salannol acetate & $\begin{array}{l}\text { Ring-C-seco-tetrano- } \\
\text { triterpenoids }\end{array}$ & Antifeeding properties & [108] \\
\hline 204 & Neem oil & Campesterol & Steroids & $\begin{array}{l}\text { Cholesterol lowering } \\
\text { anticarcinogenic and } \\
\text { angiogenesis activity }\end{array}$ & {$[109,110]$} \\
\hline 205 & Neem oil & Sitosterol & Steroids & $\begin{array}{l}\text { Anthelminthic and } \\
\text { antimutagenic activities }\end{array}$ & {$[109,111]$} \\
\hline 206 & Neem oil & Fucosterol & Steroids & $\begin{array}{l}\text { Cytotoxicity against breast } \\
\text { and colon carcinoma cell line }\end{array}$ & {$[109,112]$} \\
\hline 207 & Neem oil & $\begin{array}{l}\text { 9-octadecanoic acid-hexa- } \\
\text { decanoic acid-tetrahydro- } \\
\text { furan-3,4-diyl ester }\end{array}$ & Fatty acids and their derivatives & Antibacterial & [113] \\
\hline 208 & Seed extract & 7-decacetyl-7-benzoylgedunin & Tetranortriterpenoids & $\begin{array}{l}\text { Cytotoxic activity against } \\
\text { HL60 leukemia cells }\end{array}$ & {$[91,114]$} \\
\hline 209 & Seed extract & $\begin{array}{l}\text { 7-desacetyl-7- benzoyl- } \\
\text { epoxyazadiradione }\end{array}$ & Tetranortriterpenoids & $\begin{array}{l}\text { Cytotoxic activity against } \\
\text { HL60 leukemia cells }\end{array}$ & {$[91,114]$} \\
\hline 210 & Seeds & Vilasinin & Tetranortriterpenoids & & {$[22,43]$} \\
\hline 211 & Seeds & 1,3-di-O-acetylvilasinin & Tetranortriterpenoids & Insect antifeeding activity & {$[22]$} \\
\hline 212 & Seeds & 1-O-tigloyl-3-O-acetylvilasinin & Tetranortriterpenoids & Insect antifeeding activity & [115] \\
\hline 213 & Seeds & Limbocinin & Tetranortriterpenoids & n.a. & [116] \\
\hline 214 & Seeds & Limbocidin & Tetranortriterpenoids & n.a. & [117] \\
\hline 215 & Seeds & 4-epi-nimbin & $\begin{array}{l}\text { Ring-C-seco-tetrano- } \\
\text { triterpenoids }\end{array}$ & n.a. & [117] \\
\hline 216 & Seeds & 6-deacetylnimbinal & $\begin{array}{l}\text { Ring-C-seco-tetrano- } \\
\text { triterpenoids }\end{array}$ & n. a. & [118] \\
\hline 217 & Seeds & Nimbanal (nimbinal) & $\begin{array}{l}\text { Ring-C-seco-tetrano- } \\
\text { triterpenoids }\end{array}$ & n.a. & {$[108,118]$} \\
\hline 218 & Seeds & Nimbinol & $\begin{array}{l}\text { Ring-C-seco-tetrano- } \\
\text { triterpenoids }\end{array}$ & n. a. & [118] \\
\hline 219 & Seeds & Nimbolide & $\begin{array}{l}\text { Ring-C-seco-tetrano- } \\
\text { triterpenoids }\end{array}$ & $\begin{array}{l}\text { Cytotoxic activities against } \\
\text { N1E- } 115 \text { murine neuro- } \\
\text { blastoma cells, anticancerous } \\
\text { activity against human breast } \\
\text { cancer cells }\end{array}$ & {$[107,119-122]$} \\
\hline 220 & Seeds & 28-deoxonimbolide & $\begin{array}{l}\text { Ring-C-seco-tetrano- } \\
\text { triterpenoids }\end{array}$ & $\begin{array}{l}\text { Cytotoxic activities against } \\
\text { HL60 cells }\end{array}$ & {$[114,118,123]$} \\
\hline 221 & Seeds & $\begin{array}{l}\text { Ochinin acetate } \\
\text { (ochinin-3-acetate) }\end{array}$ & $\begin{array}{l}\text { Ring-C-seco-tetrano- } \\
\text { triterpenoids }\end{array}$ & n.a. & [124] \\
\hline 222 & Seeds & Azadirachtin & $\begin{array}{l}\text { Ring-C-seco-tetrano- } \\
\text { triterpenoids }\end{array}$ & $\begin{array}{l}\text { Most potent insect antifeedant } \\
\text { and insect growth-regulating } \\
\text { agent }\end{array}$ & {$[125,126]$} \\
\hline 223 & Seeds & $\begin{array}{l}\text { 2',3'-dihydrotigloyl- } \\
\text { azadirachtol }\end{array}$ & Tetranotriterpenoids & n. a. & [127] \\
\hline 224 & Seeds & 3-isobutyrylazadirachtol & Tetranotriterpenoids & n.a. & [127] \\
\hline 225 & Seed extract & Azadirachtin G & $\begin{array}{l}\text { Ring-C-seco-tetrano- } \\
\text { triterpenoids }\end{array}$ & n. a. & {$[128,129]$} \\
\hline
\end{tabular}


- Table 1 Continued

\begin{tabular}{|c|c|c|c|c|c|}
\hline SI. No. & Plant tissue & Compounds & Compound class & Biological activity & References \\
\hline 226 & Seed extract & $\begin{array}{l}\text { 3-O-acetyl-1-O-hydroxy- } \\
\text { azadirachtol }\end{array}$ & Tetranotriterpenoids & n.a. & {$[128,129]$} \\
\hline 227 & Seeds & $\begin{array}{l}11 \alpha \text {-hydroxy-12- } \\
\text { norazadirachtin }\end{array}$ & Tetranotriterpenoids & n.a. & [130] \\
\hline 228 & Seeds & Azadirachtin D & $\begin{array}{l}\text { Ring-C-seco-tetrano- } \\
\text { triterpenoids }\end{array}$ & n.a. & {$[128,131,132]$} \\
\hline 229 & Seeds & Azadirachtin I & $\begin{array}{l}\text { Ring-C-seco-tetrano- } \\
\text { triterpenoids }\end{array}$ & n.a. & {$[128,131,133]$} \\
\hline 230 & Seeds & Azadirachtin F & $\begin{array}{l}\text { Ring-C-seco-tetrano- } \\
\text { triterpenoids }\end{array}$ & n.a. & {$[128,131,132]$} \\
\hline 231 & Seeds & $\begin{array}{l}\text { 1-O-Tigloyl-3-O-acetyl-11- } \\
\text { hydroxymeliacarpinin }\end{array}$ & Tetranortriterpenoids & n.a. & [97] \\
\hline 232 & Seeds & $11 \beta$-hydroxy-azadirachtinin & Tetranotriterpenoids & n.a. & [15] \\
\hline 233 & Seeds & 7-benzoylnimbocinol & Limonoid & Cytotoxic activity & [114] \\
\hline 234 & Seeds & 17-epiazadiradione & Limonoid & n.a. & [114] \\
\hline 235 & Seeds & $\begin{array}{l}\text { 17-epi-17-hydroxy- } \\
\text { azadiradione }\end{array}$ & Limonoid & n.a. & [114] \\
\hline 236 & Seeds & 1,3-diacetylvilasinin & Tetranortriterpenoids & n.a. & [114] \\
\hline 237 & Seeds & 7-deacetylgedunin & Tetranortriterpenoids & n.a. & [114] \\
\hline 238 & Seeds & 6-deacetylnimbin & Limonoid & n. a. & [114] \\
\hline 239 & Seeds & 20,30-dihydrosalannin & Limonoid & n. a. & [114] \\
\hline 240 & Seed oil & Salannolide & $\begin{array}{l}\text { Ring-C-seco-tetrano- } \\
\text { triterpenoids }\end{array}$ & n.a. & [134] \\
\hline 241 & Seed extract & 1-0-acetyl-7-0-tigloylvilasinin & Tetranortriterpenoids & n.a. & [115] \\
\hline 242 & Dried seeds & $\begin{array}{l}\text { 1-O-Tigloyl-3-O-acetyl-12 } \alpha \text { - } \\
\text { acetoxyvilasinin }\end{array}$ & Tetranortriterpenoids & n.a. & {$[97,135]$} \\
\hline 243 & Dried seeds & $\begin{array}{l}\text { 1-3-di-O-acetyl-12 } \alpha \text { - } \\
\text { acetoxyvilasinin }\end{array}$ & Tetranortriterpenoids & n. a. & {$[97,135]$} \\
\hline 244 & Dried seeds & $\begin{array}{l}\text { 7-O-Tigloyl-12 } \alpha- \\
\text { acetoxyvilasinin }\end{array}$ & Tetranortriterpenoids & n.a. & {$[97,135]$} \\
\hline 245 & Seeds & $\begin{array}{l}\text { 1-O-acetyl-7-O-tigloyl- } \\
\text { nimbidinin }\end{array}$ & Tetranortriterpenoids & n.a. & {$[97,135]$} \\
\hline 246 & Seeds & $\begin{array}{l}\text { 1,3-di-O-acetyl-7-O-tigloyl- } \\
12 \beta \text {-hydroxyvilasinin }\end{array}$ & Tetranortriterpenoids & n. a. & [136] \\
\hline 247 & Seeds & $\begin{array}{l}\text { 3-O-acetyl-7-O-tigloylvilasinin } \\
\text { lactone }\end{array}$ & Tetranortriterpenoids & n.a. & [137] \\
\hline 248 & Seeds & Azadirachtin K & $\begin{array}{l}\text { Ring-C-seco-tetrano- } \\
\text { triterpenoids }\end{array}$ & n.a. & [138] \\
\hline 249 & Seed & $\begin{array}{l}\text { 3-deacetyl-11-desoxy- } \\
\text { azadirachtin }\end{array}$ & $\begin{array}{l}\text { Ring-C-seco-tetrano- } \\
\text { triterpenoids }\end{array}$ & n. a. & [137] \\
\hline 250 & Seeds & 11-hydroxyazadirachtin B & $\begin{array}{l}\text { Ring-C-seco-tetrano- } \\
\text { triterpenoids }\end{array}$ & n.a. & [136] \\
\hline 251 & Seeds & 23-desmethyllimocin-B & Limonoids & n. a. & [136] \\
\hline 252 & Seeds & $\begin{array}{l}\text { 1-tigloyl-3-acetyl- } \\
\text { azadirachtinin }\end{array}$ & $\begin{array}{l}\text { Ring-C-seco-tetrano- } \\
\text { triterpenoids }\end{array}$ & n.a. & [136] \\
\hline 253 & Seeds & $\begin{array}{l}\text { 1,2-diacetyl-7-tigloyl-12- } \\
\text { hydroxyvilasinin }\end{array}$ & Tetranortriterpenoids & n. a. & [136] \\
\hline 254 & Seeds & 11-epi-azadirachtin H & $\begin{array}{l}\text { Ring-C-seco-tetrano- } \\
\text { triterpenoids }\end{array}$ & n.a. & [139] \\
\hline 255 & Seeds & $4 \alpha$-benzoylnimbandiol & Pentanortriterpenoids & n.a. & continue \\
\hline
\end{tabular}


- Table 1 Continued

\begin{tabular}{|c|c|c|c|c|c|}
\hline SI. No. & Plant tissue & Compounds & Compound class & Biological activity & References \\
\hline 256 & Seeds & $\begin{array}{l}\text { 1-Tigloyl-3-acetyl-11-hydroxy- } \\
4 \beta \text {-methylmeliacarpin } \\
\text { (11-epi-azadirachtin D) }\end{array}$ & Terpenoids & n.a. & {$[140,141]$} \\
\hline 257 & Seeds & $\begin{array}{l}\text { 1 } \alpha \text {-destigloyl-1 } \alpha \text {-benzoyl- } \\
\text { azadirachtin }\end{array}$ & $\begin{array}{l}\text { Ring-C-seco-tetrano- } \\
\text { triterpenoids }\end{array}$ & n.a. & {$[76]$} \\
\hline 258 & Oil, seeds & Glyceride of $\alpha$-Linolenic acid & Fatty acids and their derivatives & n.a. & {$[32]$} \\
\hline 259 & Seed kernels & Nimbidic acid & $\begin{array}{l}\text { Ring-C-seco-tetrano- } \\
\text { triterpenoids }\end{array}$ & n.a. & {$[142,143]$} \\
\hline 260 & Seed kernels & $\begin{array}{l}\text { 1, 2-dihydro- } 4 \alpha, 6 \alpha \text {-dihydroxy- } \\
\text { A-homozadirone }\end{array}$ & Tetranortriterpenoids & n.a. & {$[15,144]$} \\
\hline 261 & Seed kernels & Nimbin & $\begin{array}{l}\text { Tetranortriterpenoids } \\
\text { (meliacins/limonoids) }\end{array}$ & Insecticidal activity & {$[12]$} \\
\hline 262 & Seed kernels & Azadirachtin M & $\begin{array}{l}\text { Ring-C-seco-tetrano- } \\
\text { triterpenoids }\end{array}$ & n.a. & {$[145]$} \\
\hline 263 & Seed kernels & Azadirachtin N & $\begin{array}{l}\text { Ring-C-seco-tetrano- } \\
\text { triterpenoids }\end{array}$ & n.a. & {$[145]$} \\
\hline 264 & Seed kernels & $\begin{array}{l}\text { Azadirachtin B (3-tigloyl- } \\
\text { azadirachtol) }\end{array}$ & Tetranotriterpenoids & $\begin{array}{l}\text { Insect growth regulating } \\
\text { properties }\end{array}$ & {$[58,146-149]$} \\
\hline 265 & Seed kernels & Nimbidinin & Tetranortriterpenoids & n.a. & {$[142,143]$} \\
\hline 266 & Neem kernel & 13,14-desepoxyazadirachtin-A & $\begin{array}{l}\text { Tetranortriterpenoid, ring-C- } \\
\text { seco-tetranotriterpenoids }\end{array}$ & n.a. & {$[150]$} \\
\hline 267 & Seed kernels & Salannolactam-21 & Nitrogen containing Limonoids & Antifeedant activity & {$[151]$} \\
\hline 268 & Seed kernels & Salannolactam-23 & Nitrogen containing Limonoids & Antifeedant activity & {$[151]$} \\
\hline 269 & Seed kernels & Azadirachtin $\mathrm{H}$ & $\begin{array}{l}\text { Ring-C-seco-tetrano- } \\
\text { triterpenoids }\end{array}$ & $\begin{array}{l}\text { Insect antifeedant and } \\
\text { ecdysis-inhibiting activity }\end{array}$ & {$[131-133]$} \\
\hline 270 & $\begin{array}{l}\text { Neem kernel } \\
\text { extract }\end{array}$ & Limbonin & Trinortriterpenoids & n.a. & {$[152,153]$} \\
\hline 271 & $\begin{array}{l}\text { Methanolic } \\
\text { extract of air- } \\
\text { dried kernels }\end{array}$ & $\begin{array}{l}\text { 1-benzoyl-3-deacetyl-1- } \\
\text { detigloyl salannin }\end{array}$ & Limonoids & n.a. & {$[154]$} \\
\hline 272 & $\begin{array}{l}\text { Methanolic } \\
\text { extract of air- } \\
\text { dried kernels }\end{array}$ & 7-tigloyl-12-oxo vilasini & Limonoids & n.a. & {$[154]$} \\
\hline 273 & $\begin{array}{l}\text { Methanolic } \\
\text { extract of air- } \\
\text { dried kernels }\end{array}$ & Azadiralactone & Limonoids & n.a. & {$[154]$} \\
\hline 274 & $\begin{array}{l}\text { Methanolic } \\
\text { extract of air- } \\
\text { dried kernels }\end{array}$ & Azadirahemiacetal & Triterpenoid & n.a. & {$[154]$} \\
\hline 275 & Seed oil & Nimbinene & Pentanortriterpenoids & n.a. & {$[22]$} \\
\hline 276 & Seed oil & 6-deacetylnimbinene & Pentanortriterpenoids & n.a. & {$[22]$} \\
\hline 277 & Seed oil & 6-O-acetylnimbandiol & Pentanortriterpenoids & n.a. & {$[22,105]$} \\
\hline 278 & Fresh twigs & $\begin{array}{l}\text { 6,8-dihydroxy-3-methyl-3,4- } \\
\text { dihydroisocoumarin }\end{array}$ & Coumarin and chalcones & n.a. & [15] \\
\hline 279 & Twigs & $\begin{array}{l}\text { 7,8-dihydroxy-3-methyl-3,4- } \\
\text { dihydroisocoumarin }\end{array}$ & Coumarin and chalcones & n.a. & [15] \\
\hline 280 & Twigs & $\begin{array}{l}\text { Margocetin (3,4-dihydroxy- } \\
\text { 7-8-dimethoxy-3-methyl- } \\
\text { isocoumarin) }\end{array}$ & Coumarin and chalcones & n.a. & {$[15]$} \\
\hline 281 & Fresh twigs & $\begin{array}{l}\text { 6-methoxymellein } \\
(3,4-\text { dihydroxy-6-methoxy-3- } \\
\text { methylisocoumarin) }\end{array}$ & Coumarin and chalcones & $\begin{array}{l}\text { Inhibits the growth of several } \\
\text { fungi, yeasts and bacteria }\end{array}$ & {$[15,155,156]$} \\
\hline 282 & Twigs & $\begin{array}{l}\text { Isofraxidin (7-hydroxy-6-8- } \\
\text { dimethoxycoumarin) }\end{array}$ & Coumarin and chalcones & Cytotoxic activity & {$[15,157]$} \\
\hline
\end{tabular}


- Table 1 Continued

\begin{tabular}{|c|c|c|c|c|c|}
\hline SI. No. & Plant tissue & Compounds & Compound class & Biological activity & References \\
\hline 283 & Twigs & Isonimolide & $\gamma$-Hydroxybutenolides & n.a. & [158] \\
\hline 284 & Twigs & Isonimbolide & $\gamma$-Hydroxybutenolides & n.a. & {$[158]$} \\
\hline 285 & Twigs & $\begin{array}{l}\text { Methyl esters of eicosenoic } \\
\text { acid }\end{array}$ & Fatty acids and their derivatives & n.a. & [17] \\
\hline 286 & Twigs & Margosinolide & $\begin{array}{l}\text { Ring-C-seco-tetrano- } \\
\text { triterpenoids }\end{array}$ & n.a. & [159] \\
\hline 287 & Twigs & Isomargosinolide & $\begin{array}{l}\text { Ring-C-seco-tetrano- } \\
\text { triterpenoids }\end{array}$ & n.a. & [159] \\
\hline 288 & Twigs & 6-deacetylnimbinolide & $\begin{array}{l}\text { Ring-C-seco-tetrano- } \\
\text { triterpenoids }\end{array}$ & n.a. & [160] \\
\hline 289 & Twigs & 6-deacetyl-isonimbinolide & $\begin{array}{l}\text { Ring-C-seco-tetrano- } \\
\text { triterpenoids }\end{array}$ & n.a. & [160] \\
\hline 290 & Stem bark & $\begin{array}{l}\text { 1-O-Tigloyl-3-O-acetyl-11- } \\
\text { methoxyazadirachtinin }\end{array}$ & Tetranotriterpenoids & n.a. & [97] \\
\hline 291 & Powdered bark & $\begin{array}{l}\text { 22,23-dihydro-23 } \beta \text {-methoxy- } \\
\text { azadirachtin }\end{array}$ & Tetranotriterpenoids & п.а. & [58] \\
\hline 292 & Stem bark & Nimbosodione & Diterpenoids & n.a. & {$[161]$} \\
\hline 293 & Stem bark & Nimbisonol & Diterpenoids & n.a. & [161] \\
\hline 294 & Stem bark & Demethylnimbionol & Diterpenoids & n.a. & {$[161]$} \\
\hline 295 & Stem bark & Isomargolonone & Diterpenoids & n.a. & {$[162]$} \\
\hline 296 & Stem bark & Margolone & Diterpenoids & n.a. & [162] \\
\hline 297 & Stem bark & Margolonone & Diterpenoids & n.a. & {$[162]$} \\
\hline 298 & Stem bark & Margosolone & Diterpenoids & n.a. & [163] \\
\hline 299 & Stem bark & Margosone & Diterpenoids & n.a. & [163] \\
\hline 300 & Stem bark & Methylnimbiol & Diterpenoids & n.a. & {$[164]$} \\
\hline 301 & Stem bark & Nimbosone & Diterpenoids & n.a. & [164] \\
\hline 302 & Stem bark & Nimosone & Diterpenoids & n.a. & [164] \\
\hline 303 & Stem bark & Methylnimbionone & Diterpenoids & n.a. & [164] \\
\hline 304 & Stem bark & Nimbione & Diterpenoids & п.а. & [165] \\
\hline 305 & Stem bark & Nimbinone & Diterpenoids & n.a. & [165] \\
\hline 306 & Stem bark & Nimbionol & Diterpenoids & n.a. & [166] \\
\hline 307 & Stem bark & Nimbionone & Diterpenoids & n.a. & [166] \\
\hline 308 & Stem bark & Nimbonolone & Diterpenoids & n.a. & [167] \\
\hline 309 & Stem bark & Nimbonone & Diterpenoids & n.a. & [167] \\
\hline 310 & Stem bark & Nimbiol & Diterpenoids & n.a. & [168-171] \\
\hline 311 & Stem bark & Sugiol (7-oxoferruginol) & Diterpenoids & Anti-inflammatory activity & [168-172] \\
\hline 312 & Stem bark & Margosinone & Polyacetates & n.a. & [173] \\
\hline 313 & Stem bark & Margosinolone & Polyacetates & n.a. & [173] \\
\hline 314 & Heart Wood & 24-methylene-lophenol & Steroids & n.a. & [174] \\
\hline 315 & Trunk wood & $\begin{array}{l}\text { 1,3-di-O-acetyl-7-O-cinna- } \\
\text { moylvilasinin (nimbolin A) }\end{array}$ & Tetranortriterpenoids & n.a. & [175] \\
\hline 316 & Trunk wood & Nimbolin B & $\begin{array}{l}\text { Ring-C-seco-tetrano- } \\
\text { triterpenoids }\end{array}$ & Insect antifeeding activity & {$[175,176]$} \\
\hline 317 & Bark & Gallocatechin & Flavonoids & n.a. & {$[177]$} \\
\hline 318 & Bark & epi-catechin & Flavonoids & $\begin{array}{l}\text { Anti-inflammatory and } \\
\text { immunomodulatory }\end{array}$ & [177] \\
\hline 319 & Bark & Catechin & Flavonoids & $\begin{array}{l}\text { Anti-inflammatory and } \\
\text { immunomodulatory }\end{array}$ & [177] \\
\hline 320 & Bark & epi-gallocatechin & Flavonoids & Antioxidant activity & {$[177,178]$} \\
\hline 321 & Bark & Gallic acid & Acids and their derivatives & n.a. & [177] \\
\hline
\end{tabular}


- Table 1 Continued

\begin{tabular}{|c|c|c|c|c|c|}
\hline SI. No. & Plant tissue & Compounds & Compound class & Biological activity & References \\
\hline 322 & Bark & Fraxinellone & n.a. & Antimutagenic activity & {$[175,179]$} \\
\hline 323 & Bark & $\begin{array}{l}\text { 4,14 } \alpha \text {-dimethyl-5 } \alpha \text {-ergosta- } \\
8,24(28) \text {-diene-3 } \beta \text {-ol }\end{array}$ & Steroids & n.a. & {$[180]$} \\
\hline 324 & Bark & $\begin{array}{l}4 \alpha \text {-methyl-5 } \alpha \text {-ergosta- } \\
8,24(28) \text {-diene-3 } \beta \text {-ol }\end{array}$ & Steroids & n.a. & {$[180]$} \\
\hline 325 & Stem bark & Methyl grevillate & Acids and their derivatives & n.a. & {$[167]$} \\
\hline 326 & Stem bark & Isonimbinolide & $\begin{array}{l}\text { Ring-C-seco-tetrano- } \\
\text { triterpenoids }\end{array}$ & Antifeedant activity & {$[165,181]$} \\
\hline 327 & Root bark & Azadiricin & Diterpenoids & n.a. & {$[182]$} \\
\hline 328 & Root bark & Azadirilin & Diterpenoids & n.a. & {$[182]$} \\
\hline 329 & Root bark & Azadirin A & Diterpenoids & n.a. & {$[182]$} \\
\hline 330 & Root bark & Azadirin B & Diterpenoids & n.a. & {$[182]$} \\
\hline 331 & Root bark & Nimbocinin & Diterpenoids & n.a. & {$[182]$} \\
\hline 332 & Root bark & Nimbolicidin & $\begin{array}{l}\text { Ring-C-seco-tetrano- } \\
\text { triterpenoid }\end{array}$ & n.a. & {$[182]$} \\
\hline 333 & Root bark & Nimbocin & Hexanortriterpenoids & n.a. & {$[182]$} \\
\hline 334 & Roots & Azadirinin & Tetranortriterpenoids & n.a. & [183] \\
\hline 335 & Root bark & Margocilin & Diterpenoids & n.a. & {$[184]$} \\
\hline 336 & Root bark & Margocin & Diterpenoids & n.a. & {$[184]$} \\
\hline 337 & Root bark & Margocinin & Diterpenoids & n.a. & {$[184]$} \\
\hline 338 & Root bark & Nimbidiol & Diterpenoids & n.a. & {$[185]$} \\
\hline 339 & Root bark & Nimbilicin & Diterpenoids & n.a. & {$[182]$} \\
\hline 340 & Root bark & Nimbocidin & Diterpenoids & n.a. & {$[182]$} \\
\hline 341 & Root bark & Nimolinin & Diterpenoids & n.a. & {$[182]$} \\
\hline 342 & Root bark & Nimbilin & $\begin{array}{l}\text { Ring-C-seco-tetrano- } \\
\text { triterpenoids }\end{array}$ & n.a. & {$[182]$} \\
\hline 343 & Wood oil & Cycloeucalenol & Triterpenes & n.a. & {$[186]$} \\
\hline 344 & Wood oil & 24-methylenecycloartanol & Triterpenes & n.a. & {$[186,187]$} \\
\hline 345 & Gum exudate & D-glucose & Carbohydrates and proteins & n.a. & {$[188]$} \\
\hline 346 & Gum exudate & D-glucoronic acid & Carbohydrates and proteins & n.a. & {$[188]$} \\
\hline 347 & Gum exudate & L-arabinose & Carbohydrates and proteins & n.a. & {$[188]$} \\
\hline 348 & Gum exudate & L-fucose & Carbohydrates and proteins & n.a. & {$[188]$} \\
\hline 349 & Gum exudate & Mannose & Carbohydrates and proteins & n.a. & [189] \\
\hline 350 & Gum exudate & Xylose & Carbohydrates and proteins & n.a. & {$[189]$} \\
\hline 351 & Gum exudate & Rhamnose & Carbohydrates and proteins & n.a. & [189] \\
\hline 352 & Gum exudate & D-glucosamine & Carbohydrates and proteins & n.a. & {$[190]$} \\
\hline 353 & Gum exudate & Aldobiouronic acid & Carbohydrates and proteins & n.a. & {$[191]$} \\
\hline 354 & Gum exudate & $\begin{array}{l}\text { 4-O-(4-O-methyl- } \alpha \text {-D- } \\
\text { glucopyranosyl uronic acid)- } \\
\text { D-galactose }\end{array}$ & Carbohydrates and proteins & n.a. & {$[191]$} \\
\hline 355 & Gum exudate & Aldotriouronic acid & Carbohydrates and proteins & n.a. & {$[191]$} \\
\hline 356 & $\begin{array}{l}\text { Fruit coat-ex- } \\
\text { tract, Seed oil }\end{array}$ & Epoxyazadiradione (nimbinin), & $\begin{array}{l}\text { Tetranortriterpenoids } \\
\text { (meliacins/limonoids) }\end{array}$ & Insecticidal activity & {$[78,192]$} \\
\hline 357 & $\begin{array}{l}\text { Fruit coat-ex- } \\
\text { tract, Seed oil }\end{array}$ & Azadiradione & $\begin{array}{l}\text { Tetranortriterpenoids } \\
\text { (meliacins/limonoids) }\end{array}$ & Insecticidal activity & {$[78,100]$} \\
\hline 358 & Seeds and leaves & 1-O-cinnamoylvilasinin lactone & Tetranortriterpenoids & n.a. & {$[135]$} \\
\hline 359 & Seeds and leaves & $\begin{array}{l}\text { 1-O-tigloyl-3-O-acetylvilasinin } \\
\text { lactol }\end{array}$ & Tetranortriterpenoids & n.a. & {$[135]$} \\
\hline
\end{tabular}


- Table 1 Continued

\begin{tabular}{|c|c|c|c|c|c|}
\hline SI. No. & Plant tissue & Compounds & Compound class & Biological activity & References \\
\hline 360 & Seeds and leaves & $\begin{array}{l}\text { 1-O-senecioyl-3-O-acetyl- } \\
\text { vilasinin lactone }\end{array}$ & Tetranortriterpenoids & n. a. & [193] \\
\hline 361 & Seeds and leaves & $\begin{array}{l}\text { 1-O-senecioyl-3-O-acetyl- } \\
\text { vilasinin lactol }\end{array}$ & Tetranortriterpenoids & n.a. & [193] \\
\hline 362 & $\begin{array}{l}\text { Twigs and seed } \\
\text { kernels; seed } \\
\text { extracts }\end{array}$ & 6-deactylnimbin & $\begin{array}{l}\text { Ring-C-seco-tetrano- } \\
\text { triterpenoids }\end{array}$ & Anti-feeding properties & {$[106,160,194]$} \\
\hline 363 & $\begin{array}{l}\text { Branches and } \\
\text { leaves }\end{array}$ & $\begin{array}{l}4 \alpha \text {-hydroperoxy-6- O- } \\
\text { acetylnimbandiol }\end{array}$ & Triterpenoids & n.a. & [121] \\
\hline 364 & $\begin{array}{l}\text { Branches and } \\
\text { leaves }\end{array}$ & $\begin{array}{l}24,25 \text {-epoxy-3 } \beta \text {-hydroxy-20- } \\
\text { oxo-7-tirucallene }\end{array}$ & Triterpenoids & n.a. & [121] \\
\hline 365 & $\begin{array}{l}\text { Branches and } \\
\text { leaves }\end{array}$ & $\begin{array}{l}22,23 ; 24,25 \text {-diepoxy-3 } \beta \text { - } \\
\text { hydroxy-7-tirucallene }\end{array}$ & Triterpenoids & n.a. & {$[121]$} \\
\hline 366 & $\begin{array}{l}\text { Fresh fruit coats } \\
\text { and seeds }\end{array}$ & $\begin{array}{l}5 \alpha \text {-androsta }(13 \beta \mathrm{Me})-7 \alpha \text { - } \\
\text { acetoxy-17-oxo-4,4,8- } \\
\text { trimethyl-1,14-dien-3,16- } \\
\text { dione ( } \beta \text {-nimolactone) }\end{array}$ & Nonnanortriterpenoids & n. a. & {$[82,93]$} \\
\hline 367 & $\begin{array}{l}\text { Fresh fruit coats } \\
\text { and seeds }\end{array}$ & $\begin{array}{l}5 \alpha \text {-androsta }(13 \alpha \mathrm{Me})-7 \alpha- \\
\text { acetoxy-17-oxo-4,4,8- } \\
\text { trimethyl-1,14-dien-3,16- } \\
\text { dione ( } \alpha \text {-nimolactone) }\end{array}$ & Nonnanortriterpenoids & n.a. & {$[82,93]$} \\
\hline 368 & Neem oil & Cholesterol & Steroids & n.a. & [195] \\
\hline 369 & Neem oil & Stigmasterol & Steroids & n.a. & [195] \\
\hline 370 & $\begin{array}{l}\text { Fruit coats, } \\
\text { Flowers }\end{array}$ & Hentriacontane & Hydrocarbons & Anti-inflammatory activity & $\begin{array}{l}{[19,20,64,83,} \\
196]\end{array}$ \\
\hline 371 & $\begin{array}{l}\text { Aqueous } \\
\text { extracts of } \\
\text { powdered neem } \\
\text { leaf, flowers }\end{array}$ & Azadirone & $\begin{array}{l}\text { Tetranortriterpenoids } \\
\text { (meliacins/limonoids) }\end{array}$ & $\begin{array}{l}\text { Cytotoxic activity against a } \\
\text { panel of human cancer cell }\end{array}$ & {$[47,197,198]$} \\
\hline 372 & Leaves, oil & Meliantriol & Protolimonoids & n.a. & [199] \\
\hline 373 & $\begin{array}{l}\text { Leaves and } \\
\text { flower }\end{array}$ & Nimbaflavone & Flavonoids & n.a. & {$[61,200]$} \\
\hline 374 & $\begin{array}{l}\text { Neem oil } \\
\text { and fresh fruit } \\
\text { coatings }\end{array}$ & $\begin{array}{l}\text { 3,4-dihydro-4,4,5,8- } \\
\text { tetramethylcoumarin }\end{array}$ & Coumarin and chalcones & n. a. & {$[17,83]$} \\
\hline 375 & $\begin{array}{l}\text { Neem oil } \\
\text { and fresh fruit } \\
\text { coatings }\end{array}$ & $\begin{array}{l}\text { 3,4-dihydro-4,4,7,8-tetra- } \\
\text { methyl-coumarin-6-ol }\end{array}$ & Coumarin and chalcones & n.a. & {$[17,83]$} \\
\hline 376 & Leaves and twigs & $\begin{array}{l}\text { Scopoletin (7-Hydroxy-6- } \\
\text { methoxycoumarin) }\end{array}$ & Coumarin and chalcones & $\begin{array}{l}\text { Antithyroid and antihyper- } \\
\text { glycemic activity }\end{array}$ & {$[35,201]$} \\
\hline 377 & $\begin{array}{l}\text { Leaves, } \\
\text { fruit coats }\end{array}$ & Octadecane & Hydrocarbons & n.a. & {$[19,20,83]$} \\
\hline 378 & $\begin{array}{l}\text { Leaves, fruit } \\
\text { coats, flowers }\end{array}$ & Nonadecane & Hydrocarbons & n.a. & {$[19,20,64,83]$} \\
\hline 379 & $\begin{array}{l}\text { Fruit coats, } \\
\text { flowers }\end{array}$ & Isoazadironolide & $\gamma$-Hydroxybutenolides & n.a. & {$[61,86]$} \\
\hline 380 & $\begin{array}{l}\text { Fruit coats; } \\
\text { fresh flowers }\end{array}$ & Pentacosane & Hydrocarbons & n.a. & {$[64,83]$} \\
\hline 381 & $\begin{array}{l}\text { Leaves, flowers, } \\
\text { fruit coats }\end{array}$ & Nonacosane & Hydrocarbons & n.a. & {$[19,20,64,83]$} \\
\hline 382 & $\begin{array}{l}\text { Fruit coats; } \\
\text { fresh flowers }\end{array}$ & Heptacosane & Hydrocarbons & n. a. & {$[19,20,64,83]$} \\
\hline 383 & $\begin{array}{l}\text { Fruit coats; } \\
\text { fresh flowers }\end{array}$ & Octacosane & Hydrocarbons & n.a. & {$[19,20,64,83]$} \\
\hline
\end{tabular}


- Table 1 Continued

\begin{tabular}{|c|c|c|c|c|c|}
\hline SI. No. & Plant tissue & Compounds & Compound class & Biological activity & References \\
\hline 384 & $\begin{array}{l}\text { Fruit coats; } \\
\text { flowers }\end{array}$ & Heneicosane & Hydrocarbons & n.a. & {$[64,83]$} \\
\hline 385 & $\begin{array}{l}\text { Fruit coats, } \\
\text { flowers }\end{array}$ & Tricosane & Hydrocarbons & n.a. & {$[64,83]$} \\
\hline 386 & $\begin{array}{l}\text { Oil, seeds, } \\
\text { leaves, twigs } \\
\text { and fruits }\end{array}$ & $\begin{array}{l}\text { Glycerides and methyl esters } \\
\text { of oleic acids }\end{array}$ & Fatty acids and their derivatives & n.a. & {$[202,203]$} \\
\hline 387 & $\begin{array}{l}\text { Oil, seeds, } \\
\text { leaves, twigs } \\
\text { and fruits }\end{array}$ & $\begin{array}{l}\text { Glycerides and methyl esters } \\
\text { of stearic acids }\end{array}$ & Fatty acids and their derivatives & n.a. & {$[202,203]$} \\
\hline 388 & $\begin{array}{l}\text { Oil, seeds, } \\
\text { leaves, twigs } \\
\text { and fruits }\end{array}$ & $\begin{array}{l}\text { Glycerides and methyl esters } \\
\text { of palmitic acids }\end{array}$ & Fatty acids and their derivatives & n.a. & {$[202,203]$} \\
\hline 389 & $\begin{array}{l}\text { Oil, leaves, twigs } \\
\text { and fruits }\end{array}$ & $\begin{array}{l}\text { Glycerides and methyl esters } \\
\text { of myristic acids }\end{array}$ & Fatty acids and their derivatives & n.a. & {$[202,203]$} \\
\hline 390 & $\begin{array}{l}\text { Oil, leaves } \\
\text { and twigs }\end{array}$ & $\begin{array}{l}\text { Glycerides and methyl esters } \\
\text { of arachidic acids }\end{array}$ & Fatty acids and their derivatives & n.a. & {$[202,203]$} \\
\hline 391 & Oil and leaves & $\begin{array}{l}\text { Glycerides and methyl esters } \\
\text { of behenic acids }\end{array}$ & Fatty acids and their derivatives & n.a. & {$[202,203]$} \\
\hline 392 & Oil and leaves & $\begin{array}{l}\text { Glycerides and methyl esters } \\
\text { of lignocernoic acids }\end{array}$ & Fatty acids and their derivatives & n.a. & {$[202,203]$} \\
\hline 393 & Leaves and twigs & $\begin{array}{l}\text { Methyl esters of hexadeca- } \\
\text { trienoic acid }\end{array}$ & Fatty acids and their derivatives & n.a. & [17] \\
\hline 394 & Leaves and twigs & Methyl esters of lauric acid & Fatty acids and their derivatives & n.a. & [17] \\
\hline 395 & Oil/leaves & 3-Deacetylazadirachtin & $\begin{array}{l}\text { Ring-C-seco-tetrano- } \\
\text { triterpenoids }\end{array}$ & n.a. & {$[97,204]$} \\
\hline 396 & Oil/leaves & $\begin{array}{l}2^{\prime}, 3^{\prime} \text {-dihydrotigloyl- } \\
\text { azadirachtin }\end{array}$ & $\begin{array}{l}\text { Ring-C-seco-tetrano- } \\
\text { triterpenoids }\end{array}$ & n.a. & [97] \\
\hline 397 & Oil/leaves & $\begin{array}{l}\text { 1-detigloyl-1-isobutyl- } \\
\text { roylazadirachtin }\end{array}$ & $\begin{array}{l}\text { Ring-C-seco-tetrano- } \\
\text { triterpenoids }\end{array}$ & n.a. & [97] \\
\hline 398 & Oil/leaves & $\begin{array}{l}\text { 1-detigloyl-1-isovaleroyl- } \\
\text { Azadirachtin }\end{array}$ & $\begin{array}{l}\text { Ring-C-seco-tetrano- } \\
\text { triterpenoids }\end{array}$ & n.a. & [97] \\
\hline 399 & Oil/leaves & $\begin{array}{l}\text { 1-detigloyl-1-isocaproyl-3- } \\
\text { epoxymethacroy azadirachtin }\end{array}$ & $\begin{array}{l}\text { Ring-C-seco-tetrano- } \\
\text { triterpenoids }\end{array}$ & n.a. & [97] \\
\hline 400 & Oil/leaves & $\beta$-D-glucoside & Steroids & n.a. & [205] \\
\hline 401 & Oil/leaves & Margocidin & Steroids & n.a. & [182] \\
\hline 402 & Oil/leaves & Isorhamnetin & Flavonoids & In vitro antitumor activity & {$[15,30,206]$} \\
\hline 403 & Oil/leaves & Kaempferol & Flavonoids & $\begin{array}{l}\text { Anti-inflammatory and } \\
\text { anti-ulcer effects }\end{array}$ & {$[88,207]$} \\
\hline 404 & Oil/leaves & Myricetin & Flavonoids & Antioxidant activity & {$[88,208]$} \\
\hline 405 & Oil/leaves & Nimbilicidin & п. а. & п. a. & [182] \\
\hline
\end{tabular}

observed [221]. In the year 2012, a new tetranortriterpenoid named meliacinolin was isolated from chloroform extract of dried neem leaves, which demonstrated in vivo inhibition of $\alpha$-glucosidase and $\alpha$-amylase enzyme activities in streptozotocin-nicotinamide-induced type 2 diabetes in mice [55]. Inhibition of both these enzymes offers an effective strategy of lowering the levels of postprandial hyperglycemia that prevents the digestion of carbohydrates, offering promising potential of meliacinolin as an antidiabetic agent [55].

\section{Anti-ulcer effects}

The aqueous leaf extract of neem showed anti-ulcer properties in stressed rats by preventing mast cell deregulation and increasing the amount of adherent gastric mucus [221]. Neem leaf extract exhibited anti-ulcer activity on gastric lesions in rats by blocking acid secretion through inhibition of $\mathrm{H}^{+}-\mathrm{K}^{+}$-ATPase and by preventing oxidative damage and apoptosis [222]. 


\section{Antifertility effects}

Prolonged antifertility effects were observed by a single intrauterine administration of neem oil in female Wistar rats [223]. In another study, a single dose of $50 \mu$ l of neem oil on each side of the lumen of the vas deferens of male Wistar rats acted as a long-term male contraception [224]. The neem oil fraction NIM-76 was shown to have spermicidal activity in vivo not only in rats but also in rabbits and rhesus monkeys; NIM-76 was further found to affect the motility of sperm leading to the observed infertility [225, 226]. Aqueous extract of old and tender neem leaf was found to immobilize and kill $100 \%$ human spermatozoa within 20 s [227].

\section{Antimalarial activities}

Gedunin, a tetranortriterpenoid isolated from neem, was reported to be active against Plasmodium falciparum, the causative organism of malaria [228]. The antimalarial activity of the limonoids (meldenin, isomeldenin, nimocinol, and nimbandiol) isolated from the ethanolic extract of fresh neem tree was reported to be active against chloroquine-resistant $P$. falciparum strain K1 [229]. Schwikkard and van Heerden (2002) discussed the antimalarial activity of neem compounds such as the limonoid gedunin, meldenin, and azadirachtin [230]. NeemAzal, a commercial neem seed extract containing the limonoid azadirachtin as the main component, was found to block the activity of rodent malarial parasite, Plasmodium berghei, in its vector Anopheles stephensi [231].

\section{Antiretroviral activities}

An acetone-water extract of neem leaves was found to prevent the invasion of human lymphocytes by human immunodeficiency virus (HIV), thereby protecting the target cells without any adverse effects [232]. The acetone-water extract significantly increased CD4 cell count in HIV I or HIV II patients that also led to a substantial increase in mean body weight, hemoglobin concentration, lymphocyte differential count with no adverse effects, and abnormalities in kidney and liver function parameters [233].

\section{Antifungal activities}

Khan and Shah (1992) tested leaf extracts of $A$. indica on wheat seed mycoflora and noted considerable reduction in seed mycoflora vis-à-vis better seed germination [234]. Suresh et al. (1997) studied the antifungal activity of polar extract and the impure HPLC fractions of green leaves of $A$. indica against groundnut rust disease (causal agent Puccinia arachidis Speg.) [235]. Govindachari et al. (1998) also showed the synergistic effect of various neem terpenoids on different fungal pathogens [236]. Minimum inhibitory concentration (MIC) of neem seed extract was found to be $31 \mathrm{\mu g} / \mathrm{ml}$ against clinical isolates of dermatophytes (Trichophyton rubrum, Trichophyton mentagrophytes, and Microsporum nanum) [237]. Wang et al. (2010) reported a significant reduction in the growth of the pathogens Monilinia fructicola, Penicillium expansum, Trichothecium roseum, and Alternaria alternata by neem seed kernel extracts [238].

\section{Antibacterial activities}

Mahmoodin, a novel limonoid, isolated from neem oil, showed significant antibacterial activity against various Gram-positive and Gram-negative bacteria [71]. Aquaneem, an emulsified product prepared from the neem kernel, exhibited antibacterial activity against Aeromonas hydrophila and Pseudomonas fluorescens as well as Myxobacteria sp., which are pathogenic to fish [239]. Moreover, SaiRam and co-workers (2000) studied the antimicrobial activity of the extract NIM-76 against certain bacteria, fungi, and Poliovirus and compared the same with neem oil [240]. The results revealed that NIM-76 inhibited the growth of various bacterial pathogens tested including Escherichia coli and Kleibsiella pneumoniae. The extract also showed antifungal activity against Candida albicans and antiviral activity against Poliovirus replication in Vero cell lines. Overall, NIM-76 showed stronger anti-microbial activity as compared to the neem oil. Neem seed kernel extract was found to be active against Bacillus mycoides, $B$. thuringiensis, $B$. subtilis, Nocardia sp., and Corynebacterium fascians in in vitro assays [241]. In another study, neem mouthwash was found to show antibacterial activity against salivary levels of Streptococcus mutans and Lactobacillus [242]. Neem leaf extract gel also showed antiplaque activity [243]. Polyester/cotton blend fabric treated with neem extract was reported to have antibacterial activity against both Gram-positive (Bacillus subtilis) and Gram-negative bacteria (Proteus vulgaris) [244]. Neem oil was also found to be active against Staphylococcus aureus, Salmonella typhi, Pseudomonas aeruginosa, and Escherichia coli $[245,246]$. The tetranortriterpenoid, nimolicinol, isolated from neem, was reported to be moderately antibacterial against several human pathogenic bacteria $[93,95]$. The antibacterial activity of neem leaf extract and various phytoconstituents of neem such as alkaloids, steroids, tannins, glycosides, flavonoids, and saponins were evaluated and confirmed to have antibacterial efficacies, with crude flavonoids revealing maximum antibacterial activities [247]. 9-Octadecanoic acid-hexadecanoic acid-tetrahydrofuran-3,4-diyl ester obtained from neem oil was found active against Staphylococcus aureus, Escherichia coli, and Salmonella sp. in in vitro assays [113]. M-Octadecanoic acid-3,4-tetrahydrofuran diester, isolated from the petroleum ether extract of neem oil, also showed potent antibacterial activity [248]. Alcoholic extracts of neem leaves were found to be active against the human bacterial pathogens Bacillus pumillus, Pseudomonas aeruginosa, and Staphylococcus aureus [249].

\section{Antiviral activities}

Foliar application of neem seed oil, when compared with neem seed oil-free extract, inhibited transmission of potato virus $Y$ to sweet pepper by the green peach aphid, Myzus persicae (Sulzer) suggesting that the oil interferes with virus transmission [250]. A methanolic extract fraction of leaves of neem showed antiviral activity against the Coxsackie B group of viruses [251]. Crude aqueous extract of neem leaves was reported both in vitro and in vivo to display antiviral activity against the replication of Dengue virus type-2 [252]. Aqueous neem bark extract, at concentrations ranging from 50 to $100 \mu \mathrm{g} / \mathrm{ml}$, when pre-incubated with herpes simplex virus type 1 (HSV-1), considerably blocked its entry into cells; additionally, virions treated with the extract failed to bind to the cells, suggesting role of the extract either as an attachmentblocker or having direct anti-HSV-1 property. Furthermore, cells treated with extract also inhibited herpes simplex virus type $1 \mathrm{gly}$ coprotein-mediated cell-cell fusion and polykaryocytes formation, 
signifying an additional role of the bark extract at the viral fusion step [253]. The crude acidic extract of leaves and seeds and alkaline extract of seeds were found to show high antiviral activity against HSV-1 when compared with the well-known antiviral drug acyclovir [254].

\section{Anticarcinogenic activities}

Azadirone, a limonoidal constituent isolated from methanolic extract of neem flowers, was found to be a potent cytotoxic agent with good in vitro and in vivo activities [255]. The studies also revealed that the $\alpha, \beta$-unsaturated enone moiety, or its equivalent conjugated system of A-ring, C-7 acetyloxy/chloroacetyloxy or keto group of B-ring and the furan moiety, are the structural requirements for the potent activity of azadirone and its analogs [255]. Four prenylated flavanones, 5,7,4'-trihydroxy-8-prenylflavanone, 5,4'-dihydroxy-7-methoxy-8-prenylflavanone, 5,7,4'-trihydroxy-3',8-diprenylflavanone, and 5,7,4'-trihydroxy-3',5'-diprenylflavanone, were isolated by activity-guided fractionation from the methanolic extract of the flowers of neem, which acted as potent antimutagens against Trp-P-1 (3-amino-1,4-dimethyl$5 \mathrm{H}$-pyrido[4,3-b]indole) in the Salmonella typhimurium TA98 assay [61]. Aqueous extract of neem was found to show chemopreventive potential when given to Syrian male hamsters having 7,12-dimethylbenz[a] anthracene (DMBA) induced buccal pouch carcinogenesis by modulation of lipid peroxidation, antioxidants, and detoxification systems [256]. Pre-treatment with ethanolic neem leaf extract significantly lowered the concentration of lipid peroxides and increased antioxidant levels on induced oxidative stress by the potent gastric carcinogen $\mathrm{N}$-methyl-N'-nitro-N-nitrosoguanidine (MNNG) in male Wistar rats suggesting its chemoprotective effects [257]. Significant anticarcinogenic potential was also found in leaf extracts of $A$. indica in a tumor model system $(p<0.005$ to $p<0.001)$ [258]. Subapriya et al. (2005) suggested that the chemopreventive effects of ethanolic neem leaf extract might be mediated by the induction of apoptosis [259]. Treatment of rats by aqueous neem extracts significantly decreased the proliferating cell nuclear antigen labeling indices of colon epithelium and aberrant crypt foci, suggesting a chemopreventive role in the short-term colon carcinogenesis bioassay [260]. Nimbolide, a triterpenoid extracted from the flowers of the neem, was found to have antiproliferative activity and apoptosis-inducing property against U937, HL-60, THP1, and B16 cancer cell lines [261]. The acidic extract of leaves and neutral extract of seeds possessed anticancer activity, inhibiting Ehrlich ascites carcinoma cell line growth and $\mathrm{IC}_{50}$ values were 669.43 and $724.63 \mu \mathrm{g} / \mathrm{ml}$, respectively [254]. 7-Deacetyl-7-benzoylepoxyazadiradione, 7deacetyl-7-benzoylgeduin, and 28-deoxonimbolide exhibited potent cytotoxic activity against HL60 leukemia cells while 4 other compounds (7-benzoylnimbocinol, epoxyazadiradione, gedunin, and ohchinin acetate) exhibited cytotoxic activity against 1 or more cell lines [114]. Cytotoxic activities of nimbolide isolated from branches and leaves against HL-60 have also been reported [121]. Sulfonoquinovosyldiacylglyceride, a water-soluble constituent of dried neem leaves, showed anti-cancerous activity in human leukemic cell lines $U 937$ and $\mathrm{K} 562$ with $\mathrm{IC}_{50}$ of $9 \mu \mathrm{g} / \mathrm{ml}$ [49]. Nimbolide was shown to exert apoptotic activity in estrogen-dependent (MCF-7) and estrogen-independent (MDA-MB-231) hu- man breast cancer cell lines activating caspase-8, caspase-9, caspase-3, and cleavage of PARP [122]. Induction of apoptosis in human breast cancer cells by nimbolide ratifies its future in cancer treatment as a chemotherapeutic agent [122]. NIM-76, a volatile fraction of neem oil, was reported to have no mutagenic effects and regarded as safe concerning genotoxic potential in humans [262]. In vitro inhibition of growth of mouse sarcoma was found on treatment with neem leaf glycoprotein $(25 \mu \mathrm{g} /$ mice $/$ wk subcutaneously for 4 wks) [263]. This anti-tumor immunity inhibiting the growth of mouse sarcoma was reported to be associated with increased expression of CD69, CD44, and Ki67 on CD8+ T cells [263]. Neem leaf glycoprotein showed no toxicity to various physiological functions of Swiss mice and Sprague-Dawley rats even though type 1 cytokines increased in serum with a decrease in type 2 cytokines and total IgG content in leaf glycoprotein-treated mice [264]. Change in type 1 cytokines were associated with increased anti-tumor immunity [264]. Neem oil limonoids were found to induce caspase-dependent and apoptosis-inducing factor-mediated apoptosis, as well as autophagy in cancer cells [265].

\section{Hepatoprotective activities}

Aqueous leaf extracts of neem significantly prevented changes in the serum levels of bilirubin, protein, alanine aminotransferase, aspartate aminotransferase, and alkaline phosphatase, and prevented the histological changes, thus having an antihepatotoxic activity against the damage induced by antitubercular drugs in rats [266]. Chattopadhyay and Bandyopadhyay (2005) discussed the possible mechanism of hepatoprotective activity of neem leaf extracts against paracetamol-induced hepatic damage in rats and concluded that hepatoprotective activity was possibly due to its potent antioxidant activity [267]. Mercury-induced oxidative damage in hepatic tissues was improved with neem leaf extract through its antioxidant effects [268].

\section{Antioxidant activities}

Sithisarn et al. (2006) compared free radical scavenging activity of Siamese neem tree leaf extracts against the 2,2-diphenyl-1-picrylhydrazyl (DPPH) radical and reported that most active extract was obtained with the leaf decoction method showing antioxidant activity with half-maximal effective concentration $\left(E_{50}\right)$ of $31.4 \mu \mathrm{g} /$ $\mathrm{ml}$ [269]. In another study, significant antioxidant properties were observed in leaf and bark extracts/fractions of neem, while bark was found to possess higher phenolic content than the leaves [270].

\section{Effects on CNS}

Anxiolytic activity of leaf extracts of $A$. indica was studied in rats [271]. Neem extracts could attenuate anxiogenic and appetitesuppressant effects of stress by decreasing the brain's 5-hydroxytryptamine and 5-hydroxyindolacetic acid concentration in albino Wistar rats [272]. The pharmacotherapeutic value of neem leaves was also seen in anxiety disorders of albino Wistar rats [273].

\section{Molluscicidal activities}

Singh and co-workers (1996) showed the effect of leaf, bark, cake, neem oil, and the neem-based pesticides, achook and nimbecidine of neem, against the snails Lymnaea acuminata and Indopla- 
norbis exustus and found that pure azadirachtin was more toxic compared to synthetic molluscicides [274]. In another study, crude extracts of bark, roots, and leaves of neem at $500 \mathrm{mg} / \mathrm{kg}$ and $700 \mathrm{mg} / \mathrm{kg}$ were found lethal to edible tropical land snails Archachatina marginata and Limicolaria aurora (Jay) after exposure for $72 \mathrm{~h}$ and $48 \mathrm{~h}$, respectively [275].

\section{Insecticidal activities}

The neem tree is well known for its insecticidal properties, which has been documented in a large assortment of studies. Insect growth-regulating properties were found in 23-O-methylnimocinolide and 1, 7-O-deacetyl-23-O-methyl-7 $\alpha$-O-senecioylnimocinolide [39] belonging to $y$-hydroxybutenolides group of compounds. Siddiqui et al. (2002) reported desfurano-6- $\alpha$-hydroxyazadiradione and 22, 23-dihydronimocinol as having insecticidal activity against the fourth instar larvae of mosquito (Anopheles stephensi) [36]. Two nitrogen-containing limonoids, salannolactam-21 and salannolactam-23, have been reported from neem seed kernels, which possess antifeedant activities [151]. Various tetranortriterpenoids-meliatetraolenone, zafaral, 6a-O-acetyl-7deacetylnimocinol, meliacinol, 17- $\beta$-hydroxyazadiradione, azadironic acid, limocin-A, limocin-B, epoxyazadiradione, mahmoodin, gedunin, 7-decacetylgedunin, 1, 3-di-O-acetylvilasinin, 1-O-tigloyl-3-O-acetylvilasinin, nimbin, azadiradione, and 7-deacetylazadirone-isolated from different tissues of neem have been reported to either have insecticidal activities or insect anti-feeding activities (see $>$ Table 1 ). Additionally, azadirachtin and related compounds, such as 6-deactylnimbin, nimbolin B, salannin, 3-deacetylsalannin, salannol, and salannol acetate, have been isolated from various tissues of neem and also have insecticidal or insect anti-feeding properties. Insect growth-regulating activity was observed in desfuranoazadiradione, an octanortriterpenoid isolated from fresh fruit coats [77, 82]. Meliacinin, a dinortriterpenoid isolated from fruit coats, was found to be toxic against mosquito (Anopheles stephensi) [77,78]. $\beta$-sitosterol, a steroid, has also shown insecticidal potential $[276,277]$. Odoratone (protolimonoid), isolated from methanolic extract of fresh leaves, has demonstrated a lethal effect on the fourth instar larvae of mosquitoes (A. stephensi) [56]. Larvicidal properties of neem oil were also reported against $A$. stephensi, Culex quinquefasciatus, and Aedes aegypti [278].

Neem-based shampoos, amended with neem seed extract, are effective against all stages of head lice [279]. Extracts of neem oil [280], petroleum ether extracts of neem oil, and its 4 fractions separated by column chromatography [281] were reported to be lethal in in vitro assays against rabbit mite Sarcoptes scabiei var. cuniculi larvae. Further, octadecanoic acid-tetrahydrofuran-3,4-diyl ester isolated from an active fraction of the chloroform extract of neem oil was reported to have acaricidal in vitro activity against S. scabiei larvae [282]. Neem oil microemulsion was very effective against Sarcoptes scabie var. cuniculi larvae in vitro [283].

\section{Antifilarial activities}

Alcohol and aqueous extracts of flowers of $A$. indica showed inhibition of cattle filarial parasite Setaria cervi [284].

\section{Synthesis and biological sources of azadirachtin}

In addition to its remarkable insecticidal activity, azadirachtin also exhibits a range of other biological properties. The first complete structure of azadirachtin was elucidated by Nakanishi and coworkers in 1975 [285] using extensive NMR spectroscopy, which was further revised by Kraus in 1985 [87], who proposed a C13C14 epoxide. However, it took 22 y for azadirachtin to be produced by total synthesis [286, 287]. Initially, Veitch and co-workers discussed the probable route leading to the successful synthesis of azadirachtin [288]. This was followed by Jauch (2008) [286] and Ley et al. (2008) [287] who reported the full mechanism of complete chemical synthesis of azadirachtin. Meanwhile, another group focused on the biotechnological approaches for the production of azadirachtin; its production was reported using A. indica cell suspension cultures [289]. Further, azadirachtin biosynthesis could be induced in hairy root cultures of $A$. Indica [290], which was enhanced in hairy root cultures of $A$. indica by Satdive et al. in 2007 [291]. Production of azadirachtin in neem callus and suspension cultures has also been reported [292]. Another method of androgenic culture of $A$. indica showed increased azadirachtin production [293]. In 2012, Kusari and coworkers reported the biosynthesis of azadirachtin by an endophytic fungus, Eupenicillium parvum, isolated from neem [294].

\section{Endophytic microorganisms (endophytes)}

Endophytes are one of the predominant classes of microorganisms, which reside inside healthy tissues of host plants; endophytes include bacteria, fungi, nematodes, and viruses. Fungal endophytes (or endophytic fungi) are a dynamic and multitrophic group of microorganisms that are ubiquitous in plants thriving in every ecological niche ( $\bullet$ Fig. 3). Fungal endophytes have been found associated with algae [295], lichens [296], mosses [297], ferns [298], conifers [299], large trees [300], small trees [301], palms [302,303], mangroves [304], halophytes [305], grasses [306], marine sponges [307], and seagrasses [308] to name a few. Endophytic fungi were isolated from every plant tissue including bark, flower, leaves, petioles, root, seed, and twigs [7, 309-311]. Further, endophytic fungi are well-established producers of a plethora of bioactive compounds and extracellular enzymes such as amylase, cellulase, chitinase, chitosonase, laccase, lipase, pectinase, and protease [312-315]. Being colonizers of host tissues, the endobiome plays a crucial role in creating an extra layer of protection to their host during several adverse conditions [316, 317, 373]. They also modulate host metabolism for enhanced production of high-value secondary metabolites in medicinal plants like Withania, Coleus, Papaver; this positive modulation is a result of significant-high expression of genes and transcription factors of biosynthetic pathways [318-320]. Occasionally, few endophytic species mimic host metabolic pathways and produce host signature metabolites independently [294]. Therefore, unmatched beneficial traits of the endophytes were well recognized by research communities, and several of these endophytes have been utilized for several industrial and agricultural purposes.

\section{Fungal endophytic diversity of $A$. indica}

Following-up the cues on ethnobotanical history of neem, Rajagopal and Suryanarayanan (2000) investigated and isolated 


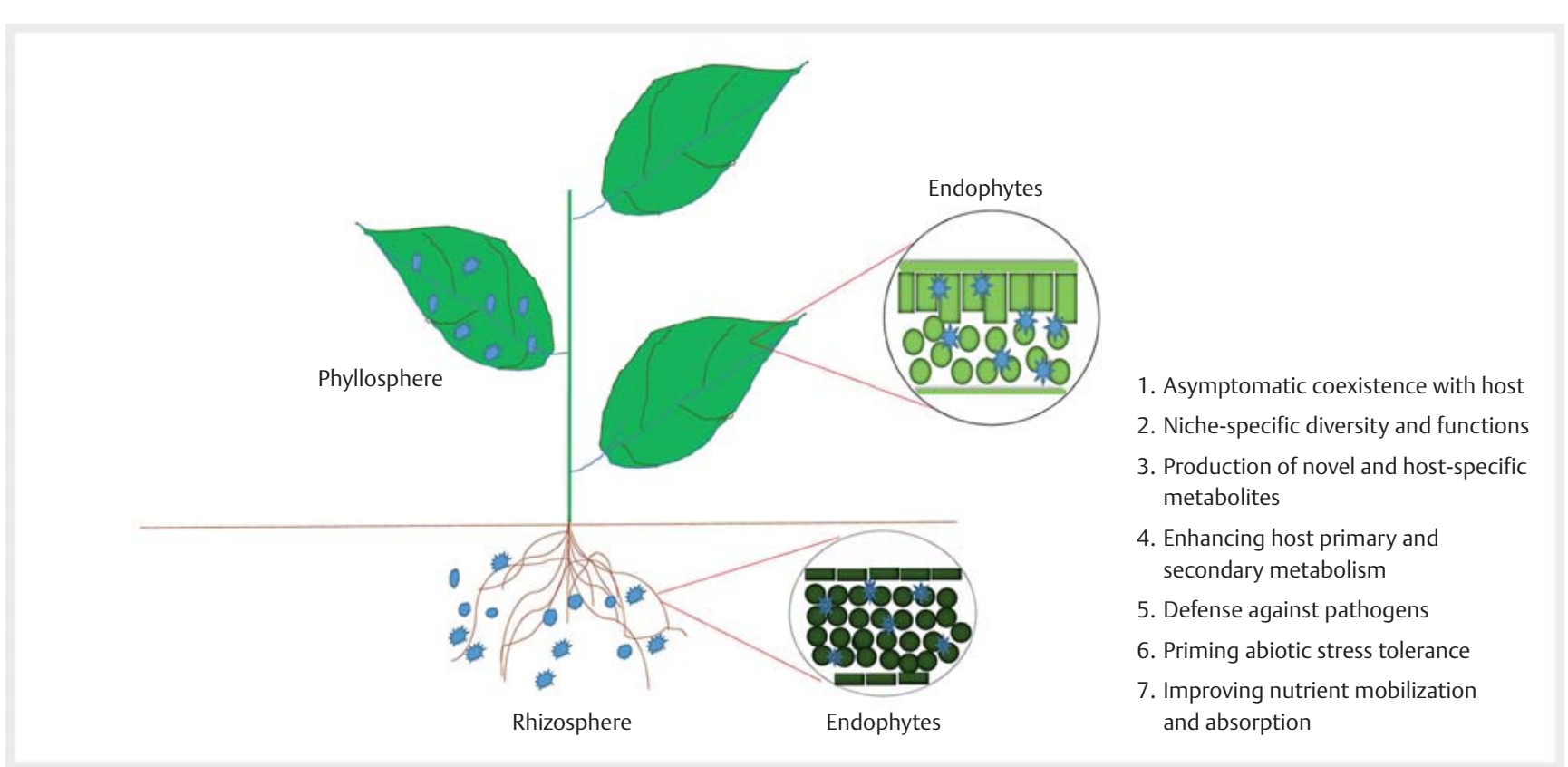

- Fig. 3 General representation of coexistence of and interaction between neem plants and associated endophytic microorganisms.

endophytic fungi from green and senescent leaves of $A$. indica from Chennai, India, continuously for 2 y on a monthly basis. They reported 5 selected endophytes, 4 of which were sterile forms and the fifth was identified as Fusarium avenaceum [321]. They proposed that the restricted number of endophytic fungal genera and the absence of common endophytic fungi in the neem leaves could be due to the antifungal metabolites present in the leaves. The frequency of colonization of green leaves by endophytes was maximal during the rainy season although no new endophyte species could be discovered. It was found that the occurrence of foliar endophytes was influenced by seasonal changes [311,322]. Since this was also found to be the case with the foliar endophytes of neem, it was suggested that the occurrence of foliar endophytes in tropical trees was influenced by the environment, soil type, and chemistry of the host tissue [321]. Mahesh and co-workers (2005) studied endophytic mycoflora harboring the inner bark of $A$. indica and reported 77 endophytic fungal isolates belonging to 15 genera [323]. Among them, 71.4\% were hyphomycetes, followed by $18.2 \%$ coelomycetes, $6.5 \%$ ascomycetes, and $3.9 \%$ sterile mycelia. The colonization frequency was found to be $38.5 \%$. Although Rajagopal and Suryanarayanan (2000) recovered only Fusarium avenaceum and 4 sterile forms of endophytes [321], Mahesh and co-workers (2005) were able to recover endophytic genera such as Curvularia, Cochlonema, Gliomastix, and Verticillium sp. [323]. Later, the same group identified endophyte diversity in bark segments of $A$. indica, which exposed high species richness with an average of 20 species, and prevalent colonization of Trichoderma and Chaetomium globosum was observed [324]. Verma and coworkers (2007) studied the fungal endophytes of $A$. indica in several of its natural habitats in India and recovered a total of 233 isolates of endophytic fungi, representing 18 fungal taxa from segments of bark, stem, and leaves [310]. Interestingly, in the whole study, the authors observed that hyphomycetes were the most prevalent group (62.2\%), followed by the coelomycetes (27.4\%) and a minor percentage by mycelia-sterilia (7.7\%). The leaf samples from all locations were nearly constant in their endophytic composition, whereas the bark samples showed maximum diversity at different locations. This study also revealed, for the first time, that endophytes of genera Periconia, Stenella, and Drechslera were associated with $A$. indica. Not only was the endophytic fungal colonization frequency higher in leaves (45.5\%) than bark (31.5\%), but the maximum species richness and frequency of colonization also were as well [310]. Shao and coworkers (2008) have studied the distribution of endophytic fungi in $A$. Indica from Yuanjiang county of Yunnan Province, PR China [325]. They isolated a total of 372 endophytic fungal strains from the stem, leaves, and fruits. Colletotrichum was found to be the most dominant genera, followed by Alternaria and Xylaria. Another group characterized 85 endophytic fungi belonging to 10 genera, which were isolated from 200 segments of fresh $A$. indica leaves collected from the Panchmarhi biosphere reserve [326]. Here, the most dominant endophytes observed were Trichoderma, Pestalotiopsis, and Penicillium sp.

Rajagopal and Suryanarayanan (2000) found that even though the endophytic genera Phomopsis, Phyllosticta, and Xylaria are ubiquitous and commonly isolated from many hosts, these were absent from the leaves of the neem plants under their study [321]. However, these genera were found to be endophytic in neem leaves by other studies [310,323,325]. Dominant endophytes fungi isolated from the inner bark of $A$. indica from South India were Trichoderma, Penicillium, and Pestalotiopsis spp. [323], while those from North India were typically Phomopsis oblonga, Cladosporium cladosporioides, Pestalotiopsis sp., Trichoderma sp., and Aspergillus sp. [310]. Further, isolated species had exhibited inhibitory properties against Trichophyton, Microsporum [327]. In China, Colletotrichum was reported as the most dominant genus, 
followed by Alternaria and Xylaria [325]. This clearly indicates that endophyte diversity and species richness are not only dependent on specific hosts but also are location and niche specific. This further illustrates the importance of sampling different tissues of a given plant at several locations to obtain an enormous species diversity of endophytes. Taken together, endophytic fungal diversity in neem has been found to be highest in stems (42\%), followed by bark (20\%), while leaves and fruits harbor a similar percentage of endophytic fungi (19\%) ( $\triangleright$ Fig. 4). With the isolation of endophytic fungi from roots and fruits of neem, in addition to previous isolation and characterization from leaves, stems and bark, Verma and colleagues completed sampling of all organs of selected neem trees for their endophytic microflora [7,300,303]. Overall, a unique diversity pattern emerges from these studies: endophytic fungi isolated from $A$. indica belong mostly to the hyphomycetes, followed by coelomycetes and finally, ascomycetes [310, 313, 321, 323, 325].

\section{Endophytic actinomycetes of $A$. indica}

In addition to endophytic fungi, neem plants have been studied for the presence of associated endophytic actinomycetes. Kharwar and coworkers characterized 55 endophytic actinomycetes from 20 different samples, $60 \%$ of which showed in vitro inhibitory activity against 1 or more pathogenic fungi or bacteria [313]. Actinomycetes were most commonly recovered from roots (54.5\% of all isolates), followed by stems (23.6\%), and finally, leaves (21.8\%). The dominant genus was Streptomyces $(49.09 \%$ of all isolates), while Streptosporangium (14.5\%), Microbispora (10.9\%), Streptoverticillium (5.5\%), Sacchromonospora sp. (5.5\%), and Nocardia (3.6\%) were also isolated. In another study, Gohain and coworkers identified the actinomycetes diversity of 6 medicinal plants collected from Gibbon wildlife sanctuary, Assam, and revealed that $A$. indica possesses the high Shannon diversity index (1.49) with predominance of Streptomyces species and Streptomyces significantly expressed Polyketide synthase-II (PKS) gene [328]. Endophytic actinomycetes species isolated from $A$. indica improved plant growth of tomato through the production of siderophores and Indole acetic acid, and inhibited the growth of the pathogen Alternaria alternata that causes blight disease in tomato [324]. Further, an actinomycete Micromonospora costi has been isolated from $A$. indica from Thailand. The unique characteristics of this species include the presence of meso-diaminopimelic acid in peptidoglycan and the presence of phospholipids like diphosphatidylglycerol, phosphatidylethanolamine, and phosphatidylinositol in the plasma membrane [329].

\section{Metabolomics of Endophytes}

In 1993, the landmark discovery of biosynthesis of the anticancer compound paclitaxel (Taxol) by endophytic Taxomyces andreanae [330] captured the attention of the scientific community towards endophytes as a treasure trove of novel, unique, bioactive natural products. A considerable number of discoveries followed the remarkable work, which cemented the virtually inexhaustible biosynthetic capabilities of endophytic fungi. Some important compounds produced by endophytic fungi are antifungal compounds such as cryptocandin A [331], cryptocin [332], ambuic acid [333,

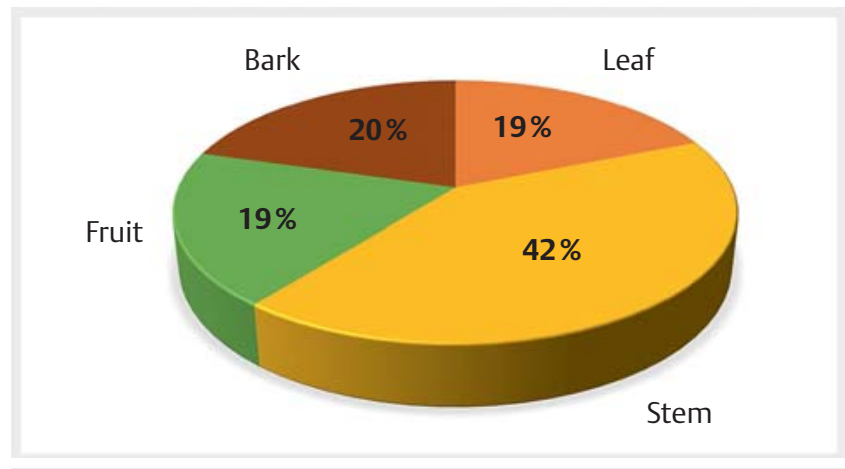

- Fig. 4 Percentage of the endophytic fungi isolated from different parts of neem.

334], pestaloside [335] and jesterone [336]; antibacterial compounds such as cytosporone A [337,338] and javanicin [314]; anticancer compounds such as torreyanic acid [339], vincristine [340], chaetoglobosin A [341], penicillenols A1 and B1 [342], and camptothecin [343]; antioxidants like pestacin [344] and isopestacin [345]; and immunosuppressant subglutinols $A$ and $B$ [346] and HIV-1 integrase inhibitors [347]. Several reviews exemplify the vast chemical diversity of compounds produced by endophytes isolated from various plants prospected from different parts of the world [313,348,349]. Recently, Chutulo et al. (2018) briefly reported the metetabolites produced by endophytes isolated from neem plant and their activities [350]. The bioactive compounds produced by endophytes not only have an ecological significance but also provide a scientific handle to study the biochemical and molecular blueprints associated with their production [351]. Herein, we present detailed elaboration on the recent developments in compounds identified from the endophytic fungi of neem plant.

\section{Bioactive natural compounds of endophytic fungi isolated from $A$. indica}

Over 30 compounds have already been reported to be produced by neem-associated fungal endophytes. For instance, chlorinated oxazinane derivatives, 10-membered lactones, solanapyrone analogues, naphthaqinones, anthraquinones, napthodianthrone derivatives, and ring-C-seco-tetranortriterpenoids are some of the essential compound classes reported to be biosynthesized by endophytes associated with neem ( $\bullet$ Fig. $\mathbf{5}$ and Table 2 ).

Two new chlorinated, epimeric 1,3-oxazinane derivatives possessing nematicidal activity were characterized from Geotrichum sp. residing endophytically in leaves of neem [352], namely 1ethanone (1) and 1-[(2R*,4S*,5R*)-2-chloro-4-methyl-1,3-oxazinan-5-yl]ethanone (2), an epimer of the first. Another nematicide active against the nematodes Bursaphelenchus xylophilus and Panagrellus redivivus, identified as [2,3-dihydro-2-(1-methylethenyl)-1-benzofuran-5-yl]methanol (3), was also reported from Geotrichum sp. in addition to 1-(2,4-dihydroxyphenyl)-ethanone (4) [352]. Ten-membered lactones viz. 8a-acetoxy-5a-hydroxy-7oxodecan-9-olide (5), 7a,8 $\alpha$-dihydroxy-3,5-decadien-10-olide (6), $7 \alpha$-acetoxymultiplolide A (7), 8 $\alpha$-acetoxymultiplolide A (8), and multiplolide A (9) have been reported from Phomopsis sp. isolated 


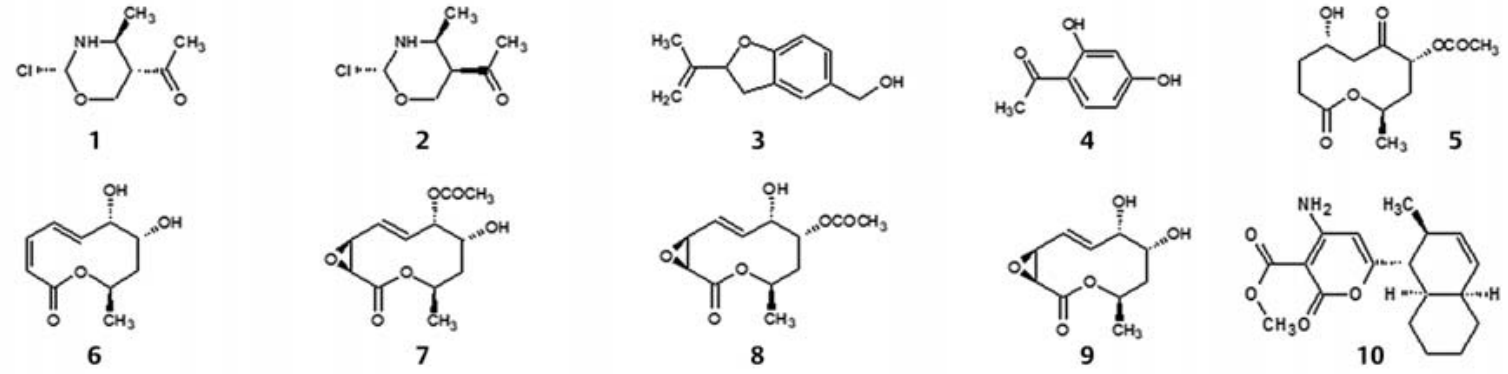

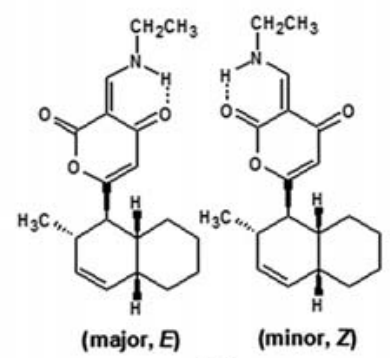

11

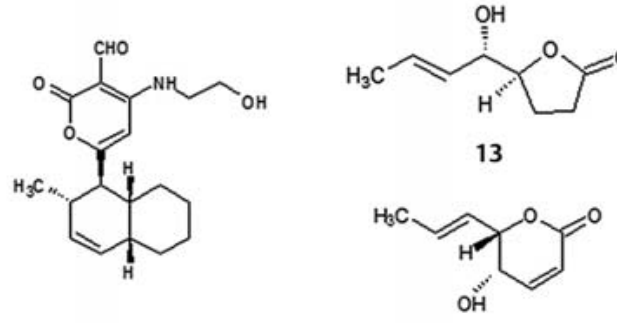

12

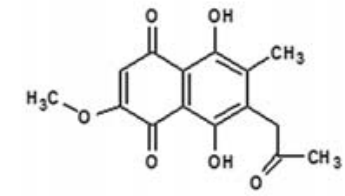

15<smiles>Cc1cc(O)c2c(c1)C(=O)c1cccc(O)c1C2=O</smiles><smiles>Cc1cc(O)c2c(c1)C(=O)c1cc(O)cc(O)c1C2=O</smiles>

17<smiles>O=C(O)CCC(=O)O</smiles>

18
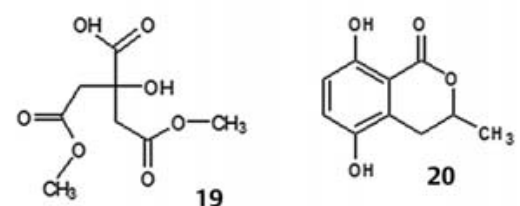<smiles>[2H]C1(C)Cc2c(C)ccc(O)c2C(=O)O1</smiles><smiles>CC1Cc2c(C(=O)O)ccc(O)c2C(=O)O1</smiles>

22

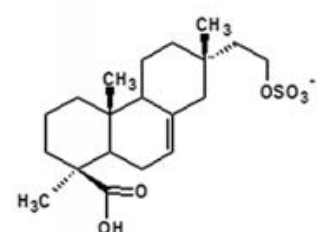

23

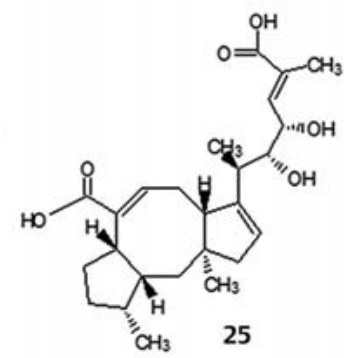

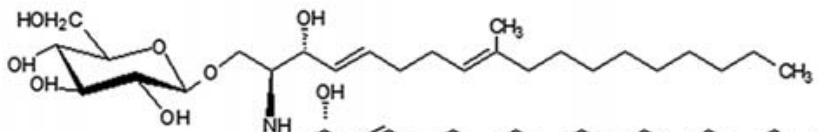

26

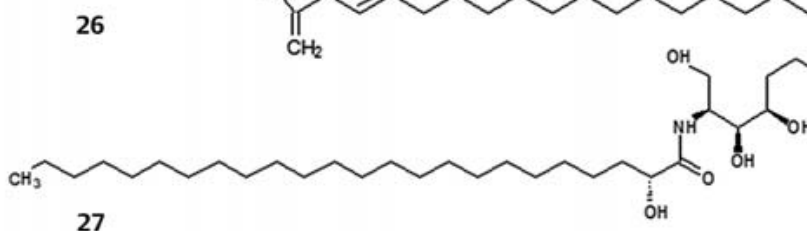

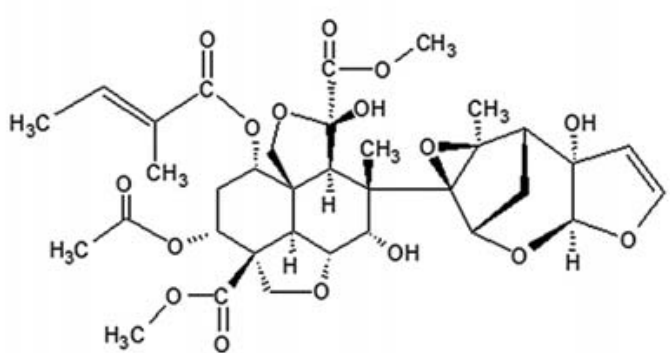

30

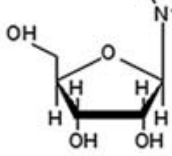

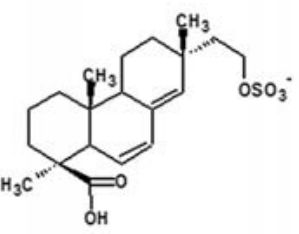

24 
- Table 2 Major bioactive compounds derived from endophytic fungi of Azadirachta indica.

\begin{tabular}{|c|c|c|c|c|c|}
\hline SI. No. & Compound & Derivative & Activity & Endophytic fungi & Reference \\
\hline 1 & $\begin{array}{l}\text { 1-[(2R*,4S*,5S*)-2-chloro-4-methyl- } \\
\text { 1,3-oxazinan-5-yl]ethanone (1) }\end{array}$ & $\begin{array}{l}\text { Chlorinated oxazinane } \\
\text { derivate }\end{array}$ & Nematicidal & Geotrichum sp. & [352] \\
\hline 2 & $\begin{array}{l}\left.\text { 1-[(2R* }, 4 S^{*}, 5 R^{*}\right) \text {-2-chloro-4-methyl- } \\
1,3 \text {-oxazinan-5-yl]ethanone (2) }\end{array}$ & $\begin{array}{l}\text { Chlorinated oxazinane } \\
\text { derivate }\end{array}$ & Nematicidal & Geotrichum sp. & [352] \\
\hline 3 & $\begin{array}{l}\text { [2,3-dihydro-2-(1-methylethenyl)-1- } \\
\text { benzofuran-5-yl]methanol (3) }\end{array}$ & Benzofuran derivative & n.a. & Geotrichum sp. & [352] \\
\hline 4 & 1-(2,4-dihydroxyphenyl)-ethanone (4) & Polyphenol & Nematicidal & Geotrichum sp. & [352] \\
\hline 5 & $\begin{array}{l}\text { 8 } \alpha \text {-Acetoxy-5 } \alpha \text {-hydroxy-7-oxodecan- } \\
\text { 9-olide (5) }\end{array}$ & $\begin{array}{l}\text { 10-membered lactone } \\
\text { ring }\end{array}$ & Antifungal & Phomopsis sp. & [353] \\
\hline 6 & $\begin{array}{l}\text { 7a, } \alpha \text {-Dihydroxy-3,5-decadien-10-olide } \\
\text { (6) }\end{array}$ & $\begin{array}{l}\text { 10-membered lactone } \\
\text { ring }\end{array}$ & Antifungal & Phomopsis sp. & [353] \\
\hline 7 & 7 $\alpha$-Acetoxymultiplolide A (7) & $\begin{array}{l}\text { 10-membered lactone } \\
\text { ring }\end{array}$ & Antifungal & Phomopsis sp. & [353] \\
\hline 8 & $8 \alpha$-Acetoxymultiplolide A (8) & $\begin{array}{l}\text { 10-membered lactone } \\
\text { ring }\end{array}$ & Antifungal & Phomopsis sp. & [353] \\
\hline 9 & Multiplolide A (9) & $\begin{array}{l}\text { 10-membered lactone } \\
\text { ring }\end{array}$ & Antifungal & Phomopsis sp. & [353] \\
\hline 10 & $\begin{array}{l}\text { Solanapyrone N (Methyl 4-Amino-6- } \\
{[(1 \mathrm{R}, 2 \mathrm{~S}, 4 \mathrm{aR}, 8 \mathrm{aR})-1,2,4 \mathrm{a}, 5,6,7,8,8 \mathrm{a}-} \\
\text { octahydro-2-methylnaphthalen-1-yl]-2- } \\
\text { oxo-2H-pyran-3-carboxylate) (10) }\end{array}$ & Solanapyrone analogues & Antifungal & Nigrospora sp. & [354] \\
\hline 11 & Solanapyrone O (11) & Solanapyrone analogues & Antifungal & Nigrospora sp. & {$[354]$} \\
\hline 12 & Solanapyrone C (12) & Solanapyrone analogues & Antifungal & Nigrospora sp. & [354] \\
\hline 13 & Nigrosporalactone (13) & Lactones & Antifungal & Nigrospora sp. & [354] \\
\hline 14 & Phomalactone (14) & Lactones & Antifungal & Nigrospora sp. & {$[354]$} \\
\hline 15 & Javanicin (15) & Naphthaqinone & Antibacterial & Chloridium sp. & [314] \\
\hline 16 & Chrysophanol (16) & Anthraquinone & Antibacterial & Aspergillus aculeatus & {$[355,356]$} \\
\hline 17 & Emodin (17) & $\begin{array}{l}\text { Napthodianthrone } \\
\text { derivative }\end{array}$ & $\begin{array}{l}\text { Antibacterial, } \\
\text { anticancerous }\end{array}$ & Aspergillus aculeatus & $\begin{array}{l}{[355,357,} \\
358]\end{array}$ \\
\hline 18 & Succinic acid (18) & Dicarboxylic acid & $\begin{array}{l}\text { Weak insecticidal activity } \\
\text { against Plutella xylostella }\end{array}$ & $\begin{array}{l}\text { Aspergillus aculeatus } \\
\text { and Xylaria sp. }\end{array}$ & {$[355,359]$} \\
\hline 19 & 1,5-Dimethyl citrate (19) & Oxobutanoate & n. a. & Aspergillus aculeatus & {$[352]$} \\
\hline 20 & 5-hydroxymellein (20) & Isocoumarin & $\begin{array}{l}\text { Weak insecticidal activity } \\
\text { against Plutella xylostella }\end{array}$ & Xylaria sp. & [359] \\
\hline 21 & 5-methylmellein (21) & Isocoumarin & $\begin{array}{l}\text { Weak insecticidal activity } \\
\text { against Plutella xylostella }\end{array}$ & Xylaria sp. & [359] \\
\hline 22 & 5-carboxymellein (22) & Isocoumarin & $\begin{array}{l}\text { Weak insecticidal activity } \\
\text { against Plutella xylostella }\end{array}$ & Xylaria sp. & [359] \\
\hline 23 & Hymatoxin C (23) & Diterpene & $\begin{array}{l}\text { Weak insecticidal activity } \\
\text { against Plutella xylostella }\end{array}$ & Xylaria sp. & [359] \\
\hline 24 & Hymatoxin D (24) & Diterpene & $\begin{array}{l}\text { Weak insecticidal activity } \\
\text { against Plutella xylostella }\end{array}$ & Xylaria sp. & [359] \\
\hline 25 & Halorosellinic acid (25) & $\begin{array}{l}\text { Ophiobolane } \\
\text { sesterterpene }\end{array}$ & $\begin{array}{l}\text { Weak insecticidal activity } \\
\text { against Plutella xylostella }\end{array}$ & Xylaria sp. & [359] \\
\hline 26 & Cerebroside C (26) & Sphingolipids & $\begin{array}{l}\text { Weak insecticidal activity } \\
\text { against Plutella xylostella }\end{array}$ & Xylaria sp. & [359] \\
\hline 27 & $\begin{array}{l}\left(2 S, 3 S, 4 R, 2^{\prime} R\right)-2-\left(2^{\prime} \text {-Hydroxytetracosa- }\right. \\
\text { noylamino)-octadecane-1,3,4-triol (27) }\end{array}$ & Ceramides (Lipids) & $\begin{array}{l}\text { Weak insecticidal activity } \\
\text { against Plutella xylostella }\end{array}$ & Xylaria sp. & [359] \\
\hline 28 & Cerevisterol (28) & Steroids & $\begin{array}{l}\text { Weak insecticidal activity } \\
\text { against Plutella xylostella }\end{array}$ & Xylaria sp. & [359] \\
\hline 29 & Adenosine (29) & Purine nucleoside & $\begin{array}{l}\text { Weak insecticidal activity } \\
\text { against Plutella xylostella }\end{array}$ & Xylaria sp. & $\begin{array}{l}\text { [359] } \\
\text { continued }\end{array}$ \\
\hline
\end{tabular}


- Table 2 Continued

\begin{tabular}{|l|l|l|l|l|l|}
\hline SI. No. & Compound & Derivative & Activity & Endophytic fungi & Reference \\
\hline 30 & Azadirachtin A (30) & $\begin{array}{l}\text { Ring-C-seco-tetrano- } \\
\text { triterpenoids }\end{array}$ & Insecticidal activity & $\begin{array}{l}\text { Eupenicillium } \\
\text { parvum }\end{array}$ & {$[294]$} \\
\hline 31 & Azadirachtin B (31) & $\begin{array}{l}\text { Ring-C-seco-tetrano- } \\
\text { triterpenoids }\end{array}$ & Insecticidal activity & $\begin{array}{l}\text { Eupenicillium } \\
\text { parvum }\end{array}$ & [294] \\
\hline
\end{tabular}

n. a.: not available

from stems of $A$. indica. These compounds exhibited antifungal activities against Aspergillus niger, Botrytis cinerea, Fusarium avenaceum, Fusarium moniliforme, Helminthosporium maydis, Penicillium islandicum, and Ophiostoma minus [353]. Multiplolide A (9), previously isolated from the fungus Xylaria multiplex [360], was also isolated from endophytic Phomopsis sp. associated with neem [353].

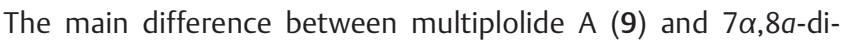
hydroxy-3,5-decadien-10-olide (6) is that the epoxide moiety at C-3 and C-4 in the former is substituted by a double bond in the latter [360]. Solanapyrones have been previously reported as phytotoxins from Ascochyta rabiei [361-363] and Alternaria solani [364, 365]. Interestingly, 2 analogs solanapyrone N (10) and solanapyrone $\mathrm{O}$ (11) were isolated from Nigrospora sp. recovered from stems of $A$. indica, with both being structurally different in the substitution pattern of the $a$-pyrone unit compared to other solanapyrones [354]. Solanapyrone N (10), solanapyrone O (11), solanapyrone $C$ (12), nigrosporalactone (13), and phomalactone (14) were shown to possess antifungal activities [354]. Structurally-related analogs of solanapyrones have also been isolated from an unidentified marine fungus associated with the surface of the green alga Halimeda monile, which demonstrated anti-algal activity [366]. Wu et al. [367] isolated guanine sesquiterpenes and isopimarane diterpenes from Xylaria sp. isolated from $A$. indica, and these compounds have shown inhibitory activities against Candida albicans, Hormodendrum compactum, and Pyricularia oryzae with MIC values ranging between $16 \mu \mathrm{g}$ to $256 \mu \mathrm{g} / \mathrm{ml}$. Similarly, 5 new guanine sesquiterpenes were further isolated from Xylaria sp. which also possesses antipathogenic activities [368]. Recently, Chatterjee et al. [369] identified the metabolites produced by Alternaria alternata isolated from $A$. indica showing inhibitory activities against Gram-negative and Gram-positive bacteria.

The highly functionalized antibacterial naphthaquinone, javanicin (15), has been reported from an endophytic fungus Chloridium sp. isolated from roots of $A$. indica [314], which displayed strong inhibition of Pseudomonas aeruginosa and $P$. fluorescens. Chrysophanol (16), emodin (17), succinic acid (18), and 1,5-dimethyl citrate (19) were obtained from the broth extract of an endophytic fungus Aspergillus aculeatus, a resident of $A$. indica [355]. Chrysophanol (1,8-dihydroxy-3-methylanthracenedione) (16), an anthraquinone responsible for antimicrobial efficacy against Bacillus subtilis and Staphylococcus aureus, was detected in the extract of Colubrina greggii [356]. Emodin (17) and related compounds were previously described as having significant inhibitory activities against P-388 leukemia in mice [357]. Emodin (17), postulated as the primary precursor in the endophytic biochemical pathway to the naphthodianthrone derivative hypericin, also showed antimicrobial activity against the Gram-positive bacterium Staphylococcus aureus, Gram-negative bacteria Klebsiella pneumoniae f. sp. ozaenae, Pseudomonas aeruginosa, Salmonella enterica f. sp. enterica, and Escherichia coli, and fungal organisms Aspergillus niger and Candida albicans [358]. Eleven compounds, namely 5-hydroxymellein (20), 5-methylmellein (21), 5-carboxymellein (22), hymatoxin $C(23)$, hymatoxin $D(24)$, halorosellinic acid (25), cerebroside $C(26),\left(2 S, 3 S, 4 R, 2^{\prime} R\right)-2-\left(2^{\prime}\right.$-hydroxytetracosanoylamino)-octadecane-1,3,4-triol (27), cerevisterol (28), adenosine (29), and succinic acid (18) have been reported to be produced by endophytic Xylaria sp. YC-10 isolated from the stems of $A$. indica collected in Yuanjiang County, Yunnan Province, P. R. China [359]. Although all the compounds exhibited weak insecticidal activity against Plutella xylostella, 9 of these compounds were reported from Xylaria for the first time [359]. Further, Verma et al. [370] attempted to synthesize silver nanoparticles from the extracts of endophytic fungus Aspergillus clavatus and tested against human pathogens such as Candida albicans, Pseudomonas fluorescens, and Escherichia coli, and they were effective against pathogens at $9.7 \mu \mathrm{g} / \mathrm{ml}$ (minimum fungicidal concentration) and $5.83 \mu \mathrm{g} / \mathrm{ml}$ (minimum inhibitory concentration). Kusari et al. (2012) identified and quantified azadirachtin A (30) and B (31) as biosynthetic products of a novel neem-associated endophytic fungus, Eupenicillium parvum [294]. This study highlighted an interesting plant-endophyte association where plant "mimetic" compounds are produced by endophytes to render similar functional traits in their ecological habitats.

\section{Outlook}

A. indica (neem) and its endophytic microflora represent an extensive repertoire of diverse natural products having different biological activities. On the one hand, neem (host plant) is a rich source of compounds such as the azadirachtins and related tetranortriterpenoids. On the other hand, endophytes associated with neem have a massive potential in synthesizing bioactive and chemically novel compounds. It is noteworthy that a large number of diverse endophytic fungi and actinomycetes have been isolated from A. indica in a relatively small period. Concomitantly, a large number of compounds have been isolated from neem and its endophytes, even though their biochemistry and overall ecological connotations are not clearly understood. Except for a few studies, endophytic microorganisms of neem remain poorly investigated. Recently, an epigenetic study was conducted to induce the anti- 
microbial activity and production of cryptic metabolites from Streptomyces coelicolor (AZRA 37) of neem plant, and the increased antimicrobial activity coupled with induced protein production were registered [371]. Extensive research is required to assess the hidden endophytic populations of neem. In particular, endophytic actinomycetes associated with neem can serve as a precious and reliable resource of novel compounds, given that they are well-known prolific producers of bioactive metabolites [372]. It has already been firmly established that endophytes have unique functions in hosts such as plant protection, nutrient supply, phosphate solubilization, and mineral transport. Besides, endophytic fungi can also confer a profound impact on the host system by not only enhancing growth and fitness but also strengthening their tolerances to abiotic and biotic stresses. It has been proposed that during evolution, some co-existing endophytes and their host plants have established a unique relationship with one another and significantly influenced the formation of secondary metabolites in plants such as neem. These findings open new platforms for enhancing growth as well as for improved production of valuable metabolites using endophytes in the host plant. However, mechanisms underlying plant-endophyte interaction are still open to future research. It is known that during endophyte infection, selected plant-specific metabolites play a significant role in colonization and the establishment of endophytic interactions. These substances not only play a crucial role in defense and competition but also might be needed for specific interaction and communication with the endophyte(s). As highlighted in this review, endophytic associations have been studied, using a bird'seye view from the host plant's side, which resulted in detailed and comprehensive knowledge related to various microbes associated with different species or cultivars. However, how the host plant (A. indica) responds varies depending on the endophyte strain and plant environment. The mechanisms behind such selective priming remains obscure. Extricating the changes in transcriptome and, subsequently, metabolome - both of neem as well as associated endophytes - under the influence of abiotic and biotic environmental factors will throw light into the genetic and biochemical mechanisms underlying neem-endophyte interactions.

\section{Acknowledgements}

RNK thankfully acknowledges the head and coordinator of CAS and FIST in Botany, BHU, Varanasi, for facilities; DST-PURSE, UGC-UPE, BHU, Varanasi, CSIR and UGC, New Delhi, for financial support and funding as JRF and SRF, and DST, New Delhi for financial support (SB/EMEQ-121/ 2014). Research in the laboratory of SK is supported by the German Federal Ministry of Education and Research (BMBF; grant no. 031B0512E); German Academic Exchange Service (DAAD); the Ministry of Innovation, Science, Research, and Technology of the State of North Rhine-Westphalia; German Research Foundation (DFG); and TU Dortmund, Germany.

\section{Conflict of Interest}

The authors declare that they have no conflict of interest.

\section{References}

[1] National Research Council. Neem: A Tree for solving global Problems. Washington, DC: National Academy Press; 1992. doi:10.17226/1924

[2] Ludwig-Muller J. Plants and endophytes: equal partners in secondary metabolite production? Biotechnol Letters 2015; 37: 1325-1334

[3] Hawksworth DL. The fungal dimension of biodiversity: magnitude, significance, and conservation. Mycol Res 1991; 95: 641-655

[4] De Bary A. Morphologie und Physiologie der Pilze, Flechten, und Myxomyceten. In: Hofmeister W. Hofmeister's Handbook of Physiological Botany, vol 2. Leipzig: W. Engelmann; 1866

[5] Wilson D. Endophyte the evolution of a term, and clarification of its use and definition. Oikos 1995; 73: 274-276

[6] Mostert L, Crous PW, Petrini O. Endophytic fungi associated with shoots and leaves of Vitis vinifera, with specific reference to the Phomopsis viticola complex. Sydowia 2000; 52: 46-58

[7] Verma VC, Gond SK, Kumar A, Kharwar RN, Boulanger LA, Strobel GA. Endophytic fungal flora from roots and fruits of an Indian neem plant Azadirachta indica A. Juss., and impact of culture media on their isolation. Indian J Microbiol 2011; 51: 469-476

[8] Roxburgh W. Description of Indian Plants. New Delhi, India: Today and Tomorrows Printers and Publishers; 1874

[9] Ahmed S. Economic, Socio-Economic and Policy Considerations on Neem's current Use for Pest Management. In: Schmutterer H, ed. The Neem Tree Azadirachta indica A. Juss. and other Meliaceous Plants. Weinheim, Germany: VCH Verlagsgesellschaft; 1995: 559-578

[10] De Jussieu A. Memoire sur le group des Meliacees. Mem Mus Hist Nat 1830; 19: 153-304

[11] Dube R. Neem Kranti. Lucknow: Shubham Prakashan; 1996

[12] Siddiqui $S$. A note on the isolation of three new bitter principles from the neem oil. Curr Sci 1942; 11: 278-279

[13] Siddiqui S, Mitra C. Utilization of neem oil and its bitter constituents (nimbidin series) in the pharmaceutical industry. J Sci Ind Res 1945; 4: $5-10$

[14] Islam M, Al-Amin M, Siddiqi MMA, Akter S, Haque MM, Sultana N, Chowdhury AMS. Isolation of quercetin-3-o-beta-d-glucopyranoside from the leaves of Azadirachta Indica and antimicrobial and cytotoxic screening of the crude extracts. Dhaka Univ J Sci 2012; 60: 11-14

[15] Siddiqui BS, Ali ST, Ali SK. Chemical Wealth of Azadirachta indica (Neem). In: Singh KK, Phogat S, Tomar A, Dhillon RS, eds. Neem. I. K. New Delhi: International Publishing House Pvt; 2009: 171-207

[16] Chen JJ, Chang YL, Teng CM, Chen IS. Anti-platelet aggregation alkaloids and lignans from Hernandia nymphaeifolia. Planta Med 2000; 66: 251256

[17] Rasheed M. Studies on the chemical Constituents of Azadirachta indica A Juss (Naeem) [PhD Dissertation]. Karachi: University of Karachi; 2002

[18] Mori M. (n-Hexacosanol and n-Octacosanol: feeding stimulants for larvae of the silkworm, Bombyx mori. J Insect Physiol 1982; 28: 969-973

[19] Schmutterer H, Ascher KRS. Natural Pesticides from the Neem Tree (Azadirachta indica A Juss) and other tropical Plants. In: Proceedings of the 2nd International Neem conference, Rauischholzhausen, Federal Republic of Germany. Eschborn, Germany: Deutsche Gesellschaft fur Technische Zusammenarbeit (GTZ) GmbH; 1983

[20] Awasthi YC, Mitra C. Constituents of Melia indica leaves. Phytochemistry 1971; 10: 2842

[21] Powell JW. Nuclear magnetic resonance spectroscopy. A correlation of the spectra of some meliacins and their derivatives. J Chem Soc C 1966; 1794-1798

[22] Kraus W, Cramer R. Neue Tetranortriterpenoide mit Insektenfrashemmender Wirkung aus Neem Oil. Liebigs Ann Chem 1981: 181-189 
[23] Nair SM, Gopal SD, Isaac D. Optimised isolation procedure for biologically active compounds nimbolide and 28-deoxonimbolide from Azadirachta indica leaves. Phytochemistry 1997; 46: 1177-1178

[24] Zhao G, Etherton TD, Martin KR, West SG, Gillies PJ, Kris-Etherton PM. Dietary alpha-linolenic acid reduces inflammatory and lipid cardiovascular risk factors in hypercholesterolemic men and women. J Nutr 2004; 134: 2991-2997

[25] Miller N], Sampson J, Candeias LP, Bramley PM, Rice-Evans CA. Antioxidant activities of carotenes and xanthophylls. FEBS Lett 1996; 384: $240-242$

[26] Chakrabortty T, Verotta L, Poddar G. Evaluation of Azadirachta indica leaf extract for hypoglycaemic activity in rats. Phytother Res 1989; 3: 30-32

[27] Verma VS. Study on the effect of flavonoids on the infectivity of potato virus X. Zentralbl Bakteriol Parasitenkd Infektionskr Hyg 1973; 128: 467472

[28] Shah W, Rane N, Kekare MB, Vaidya V. Estimation of two bioactive compounds from Azadiracta indica A. Juss. leaves using HPLC. Int ] Pharma Bio Sci 2010; 1: 1-7

[29] Deschner EE, Ruperto J, Wong G, Newmark HL. Quercetin and rutin as inhibitors of azoxymethanol-induced colonic neoplasia. Carcinogenesis 1991; 12: 1193-1196

[30] Basak SP, Chakraborty DP. Chemical investigation of Azadirachta indica leaf (Melia azadirachta). J Indian Chem Soc 1968; 45: 466-467

[31] Siddiqui S, Mahmood T, Siddiqui BS, Faizi S. Studies on the norterpenoidal constituents of Azadirachta indica. Pak J Sci Ind Res 1985; 28: 1

[32] Basu NM, Ray GK, De MK. The vitamin-C and carotene content of several herbs and flowers used in Ayurvedic system of medicine. I Indian Chem Soc $1947 ; 24: 358-360$

[33] Garg HS, Bhakuni DS. 2',3'-Dehydrosalannol, a tetranortriterpenoid from Azadirachta indica leaves. Phytochemistry 1985; 24: 866-867

[34] Kraus W, Gutzeit H, Bokel M. 1,3-diacetyl-11,19-deoxo-11-oxomeliacarpin, a possible precursor of azadirachtin from Azadirachta indica A. Juss. (Meliceae). Tetrahedron Lett 1989; 30: 1797-1798

[35] Siddiqui S, Siddiqui BS, Fiazi S, Mahmood T. Isoazadirolide, a new tetranortriterpenoid from Azadirachta indica A. Juss (Meliaceae). Heterocycles 1986; 24: 3163-3167

[36] Siddiqui BS, Afshan F, Faizi S, Naqvi SNH, Tariq RM. Two new triterpenoids from Azadirachta indica and their insecticidal activity. J Nat Prod 2002; 65: 1216-1218

[37] Siddiqui S, Siddiqui BS, Mahmood T, Fiazi S. Tetranortriterpenoid from Azadirachta indica A. Juss (Meliaceae). Heterocycles 1989; 29: 87-96

[38] Siddiqui S, Faizi S, Mahmood T, Siddiqui BS. Isolation of a new tetranortriterpenoid from Azadirachta indica A. Juss (Meliaceae). Heterocycles 1986; 24: 1319-1324

[39] Siddiqui BS, Afshan F, Ghiasuddin, Faizi S, Naqvi SNH, Tariq RM. New insect-growth-regulator meliacin butenolides from the leaves of Azadirachta indica A. Juss. J Chem Soc Perkin Trans I 1999: 2367-2370

[40] Van der Nat JM, Hart LA, Van der Sluis WG, Van Dijk H, Van den Berg AJ, de Silva KT, Labadie RP. Characterization of anticomplement compounds from Azadirachta indica. J Ethnopharmacol 1989; 27: 15-24

[41] Pachapurkar RV, Kornule PM. Tetranortriterpenoids from the leaves of Azadirachta indica. Acta Ciencia Indica 1983; 9 C: 55-59

[42] Subapriya R, Nagini S. Medicinal properties of neem leaves: A review. Curr Med Chem Anticancer Agents 2005; 5: 149-156

[43] Pachapurkar RV, Kornule PM, Narayanan CR. A new hexacyclic tetranortriterpenoid. Chem Lett 1974; 4: 357-358

[44] Siddiqui S, Faizi S, Mahmood T, Siddiqui BS. Two new insect growth regulator meliacins from Azadirachta indica A. Juss (meliaceae). J Chem Soc Perkin Trans 1 1986: 1021-1025

[45] Siddiqui BS, Afshan F, Gulzar T, Hanif M. Tetracyclic triterpenoids from the leaves of Azadirachta indica. Phytochemistry 2004; 65: 2363-2367
[46] Afshan F. Studies on the chemical constituents of the leaves of Azadirachta indica (Naeem) [PhD Dissertation]. University of Karachi: Karachi; 2002

[47] Bruhn A, Bokel M, Kraus W. 4a-6a-Dihydroxy-A-homoazadiron, ein neues Tetranortriterpenoid aus Azadirachta indica A. Juss (meliaceae). Tetrahedron Lett 1984; 25: 3691-3692

[48] Ragasa YC, Nacpil ZD, Natividad GM, Tada M, Coll JC, Rideout JA. Tetranotriterpenoids from Azadirachta indica. Phytochemistry 1997; 46: 555 558

[49] Bharitkar YP, Bhattacharya S, Das T, Roy M, Besra SE, Gomes A, Mondal NB, Banerjee S. Anti-leukemic activity of sulfonoquinovosyldiacylglyceride (SQDG): a constituent of Azadirachta indica leaves. Med Chem Res 2013; 22: 22-27

[50] Podder G, Mahato SB. Azadirachtanin, a new limonoid from the leaves of Azadirachta indica. Heterocycles 1985; 23: 2321-2325

[51] Siddiqui S, Tariq M, Bina SS, Shaheem F. Isolation of a triterpenoid from Azadirachta indica. Phytochemistry 1986; 25: 2183-2186

[52] Siddiqui S, Faizi S, Siddiqui BS. Studies on the chemical constituents of Azadirachta indica A. Juss (Meliaceae) Part I: isolation and structure of a new tetranortriterpenoid - nimolicinol. Heterocycles 1984; 22: 295-298

[53] Govindachari TR, Malathi R, Gopalakrishnan Geetha, Suresh G, Rajan SS. Isolation of a new tetranortriterpenoid from the uncrushed green leaves of Azadirachta indica. Phytochemistry 1999; 52: 1117-1119

[54] Siddiqui BS, Afshan F, Faizi S. Three novel tetracyclic triterpenoids of biogenetic interest from the leaves of Azadirachta indica. Tetrahedron 2001; 57: 10281-10286

[55] Perez-Gutierrez RM, Damian-Guzman M. Meliacinolin: a potent $a$-glucosidase and a-amylase inhibitor isolated from Azadirachta indica leaves and in vivo antidiabetic property in streptozotocin-nicotinamide-induced type 2 diabetes in mice. Biol Pharm Bull 2012; 35: 1516-1524

[56] Siddiqui BS, Afshan F, Gulzar T, Sultana R, Naqvi SN, Tariq RM. Tetracyclic triterpenoids from the leaves of Azadirachta indica and their insecticidal activities. Chem Pharm Bull 2003; 51: 415-417

[57] Siddiqui BS, Afshan F, Ghiasuddin, Faizi S, Naqvi SN, Tariq RM. Two insecticidal tetranortriterpenoids from Azadirachta indica. Phytochemistry 2000; 53: 371-376

[58] Kraus W, Bokel M, Bruhn A, Cramer R, Klaiber I, Klenk A, Nagl G, Pohnl H, Sadlo $\mathrm{H}$, Vogler B. Structure determination by NMR of azadirachtin and related compounds from Azadirachta indica A. Juss. (Meliaceae). Tetrahedron 1987: 43: 2817-2830

[59] Nanduri S, Banstola KP. Neeflone, a new tetranortriterpenoid from the flowers of Azadirachta indica A. Juss (Meliaceae). ChemInform 1996; 27: 1019-1020

[60] Siddiqui BS, Ali ST, Rasheed M, Kardar MN. Chemical constituents of the flowers of Azadirachta indica. Helv Chim Acta 2003; 86: 2787-2796

[61] Nakahara K, Roy MK, Ono H, Maeda I, Kameyama MO, Yoshida M, Trakoontivakorn G. Prenylated flavanones isolated from flowers of Azadirachta indica (the Neem Tree) as antimutagenic constituents against heterocyclic amines. J Agric Food Chem 2003; 51: 6456-6460

[62] Siddiqui BS, Tariq Ali S, Kashif Ali S. A new flavanoid from the flowers of Azadirachta indica. Nat Prod Res 2006; 20: 241-245

[63] Subramanian SS, Nair AGR. Melicitrin, a new myricetin glycoside from the flowers of Melia azadirachta. Indian J Chem 1972; 10 B: 452

[64] Siddiqui BS, Ali ST, Rajput MT, Gulzar T, Rasheed M, Mehmood R. GC-based analysis of insecticidal constituents of the flowers of Azadirachta indica A. Juss. Nat Prod Res 2009; 23: 271-283

[65] Lavie D, Jain MK, Kirson I. Terpenoids. Part VI. The complete structure of melianone. J Chem Soc 1967: 1347-1351

[66] Su RH, Kim M, Yamamoto T, Takahashi S. Antifeeding constituents of Phellodendron chinense fruit against Reticulitermes speratus. I Pesticide Sci 1990; 15: 567-572 
[67] Siddiqui S, Siddiqui BS, Faizi S, Tariq M. Studies on the chemical constituents of Azadirachta indica. A. Juss (Meliaceae) Part-VI. J Chem Soc 1986; 8: 341-347

[68] Koul O, Multani JS, Goomber S, Daniewski WM, Berlozecki S. Activity of some nonazadirachtin limonoids from Azadirachta indica against lepidopteran larvae. Australian J Entomol 2004; 43: 189-195

[69] Siddiqui S, Siddiqui BS, Faizi S. Studies in the chemical constituents of Azadirachta indica Part II: isolation and structure of the new triterpenoid azadirachtol. Planta Med 1985; 51: 478-480

[70] Malathi R, Rajan SS, Gopalakrishnan G, Suresh G. Azadirachtol, a tetranortriterpenoid from neem kernels. Acta Crystallogr C 2002; 58: 708710

[71] Siddiqui S, Faizi S, Siddiqui BS, Ghiasuddin. Constituents of Azadirachta indica: isolation and structure elucidation of a new antibacterial tetranortriterpenoid, mahmoodin, and a new protolimonoid, naheedin. J Nat Prod 1992; 55: 303-310

[72] Siddiqui S, Siddiqui BS, Ghiasuddin, Faizi S. Tetracyclic triterpenoids of the fruit coats of Azadirachta indica. J Nat Prod 1991; 54: 408-415

[73] Kraus W, Cramer R. 17-epi-Azadiradion und 17- $\beta$-hydroxy-Azadiradion, zwei neue Inhaltsstoffe aus Azadirachta indica A. Juss. Tetrahedron Lett 1978; 27: 2395-2398

[74] Nathan SS, Kalaivani K, Murugan K. Effects of neem limonoids on the malaria vector Anopheles stephensi Liston (Diptera: Culicidae). Acta Trop 2005; 96: 47-55

[75] Kraus W, Bokel M. Neue Tetranortriterpenoide aus Melia azedarach Linn. (Meliaceae). Chem Ber 1981; 114: 267-275

[76] Rojatkar SR, Sawaikar DD, Nagasampagi BA. New tetra and Pentanortriterpenoids from Azadirachta indica. India: World Neem Conference; 1993: 203

[77] Siddiqui BS, Rasheed M, Ghiasuddin, Faizi S, Naqvi SNH, Tariq RM. Biologically active triterpenoids of biogenetic interest from the fresh fruit coats of Azadirachta indica. Tetrahedron 2000; 56: 3547-3551

[78] Tariq RM, Naqvi SNH, Rasheed M, Siddiqui BS, Gabol K, Zafar SMN. Enzyme inhibition by neem fruit coat extract, two fractions and four pure compounds in treated 4th instar larvae of Anopheles stephensi Liston (O. T. Wild-strain). Pak J Entomol 2009; 24: 9-14

[79] Siddiqui S, Siddiqui BS, Ghiasuddin, Faizi S. Terpenoids from fruit coatings of Azadirachta indica. Phytochemistry 1991; 30: 1615-1619

[80] Siddiquin BS, Rasheed M. Three new triterpenoids from Azadirachta indica. Helvetica Chimica Acta 2001; 84: 1962-1968

[81] Siddiqui S, Mahmood T, Faizi S, Siddiqui BS. Studies in the chemical constituents of Azadirachta indica A. Juss (Meliaceae): Part 10. Isolation and structure elucidation of isonimolicinolide, the first 17-acetoxytetranortriterpenoid and nimolicinoic acid, the first hexanortriterpenoid with an apoeuphane (apotirucallane) Skeleton. J Chem Soc Perkin Trans I 1987: 1429-1432

[82] Siddiqui BS, Ghiasuddin, Faizi S, Siddiqui S. Triterpenoids from the fresh fruit coats of Azadirachta indica. Phytochemistry 1992; 31: 4275-4278

[83] Siddiqui BS, Rasheed M, Ilyas F, Gulzar T, Tariq RM, Naqvi SN. Analysis of insecticidal Azadirachta indica A. Juss. fractions. Z Naturforsch C 2004; 59: 104-112

[84] Siddiqui BS, Ghiasuddin, Faizi S. Tetracyclic triterpenoids of the fruit coats of Azadirachta indica. Phytochemistry 1998; 47: 1631-1636

[85] Lee JY, Lee JY, Yun BS, Hwang BK. Antifungal activity of $\beta$-asarone from rhizomes of Acorus gramineus. J Agric Food Chem 2004; 52: 776-780

[86] Siddiqui BS, Ghiasuddin G, Faizi S, Rasheed M. Triterpenoids of the fruit coats of Azadirachta indica. J Nat Prod 1999; 62: 1006-1009

[87] Kraus W, Bokel M, Klenk A, Pöhn H. The structure of azadirachtin and 22,23-dihydro-23 $\beta$-methoxyazadirachtin. Tetrahedron Lett 1985; 26: 6435-6438

[88] Luo XD, Wu SH, Ma YB, Wu DG. Four triterpenoids from neem extracts and their insecticidal activities. Nat Prod Res Dev 2001; 13: 9-13
[89] Sankaram AVB, Murthy MM, Bhaskaraiah K, Subramany AM, Sultana N, Sharma HC, Leuschner K, Ramaprasad G, Sitaramaiah S, Rukmini C, Rao PU. Chemistry, biological Activity and Utilization Aspects of some promising Neem Extractives. In: Schmutterer $\mathrm{H}$, Ascher KRS, eds. Natural Pesticides from the Neem Tree and other tropical Plants. Eschborn, Germany: German Society for Technical Cooperation (GTZ); 1986: 127-148

[90] Thakur RS, Singh SD, Goswami A. Biological and medicinal applications of Azadirachta indica A. Juss. Curr Res Med Aromat Plants 1981; 3: 135140

[91] Kraus W, Cramer R, Sawitzki G. Tetranortriterpenoids from the seeds of Azadirachta indica. Phytochemistry 1981; 20: 117-120

[92] Alam A, Haldar S, Thulasiram HV, Kumar R, Goyal M, Iqbal MS, Pal C, Dey S, Bindu S, Sarkar S, Pal U, Maiti NC, Bandyopadhyay U. Novel anti-inflammatory activity of epoxyazadiradione against macrophage migration inhibitory factor: inhibition of tautomerase and pro-inflammatory activities of macrophage migration inhibitory factor. J Biol Chem 2012; 287: 24844-24861

[93] Akihisa T, Noto T, Takahashi A, Fujita Y, Banno N, Tokuda H, Koike K, Suzuki T, Yasukawa K, Kimura Y. Melanogenesis inhibitory, anti-inflammatory, and chemopreventive effects of limonoids from the seeds of Azadirachta indicia A. Juss. (neem). J Oleo Sci 2009; 8: 581-594

[94] Hallur G, Sivramakrishnan A, Bhat SV. Three new tetranortriterpenoids from neem seed oil. J Nat Prod 2002; 65: 1177-1179

[95] Narayanan CR, Pachapurkar RV, Sawant BM, Wadia MS. Vepinin, a new constituent of neem oil. Indian J Chem 1969; 7: 187

[96] Saxena N, Kumar Y. Chemistry of Azadirachtin and other bioactive Isoprenoids from Neem (Azadiricta indica A. Juss.). In: Singh KK, Phogat S, Tomar A, Dhillon RS, eds. Neem. I. K. New Delhi: International Publishing Pvt; 2009: 208-230

[97] Kraus W, Bokel M, Schwinger M, Vogler B, Soellner R, Wendisch D, Steffens R, Wachendorff $U$. The Chemistry of Azadirachtin and other insecticidal Constituents of Meliaceae. In: Van Beek T, Breteler H, eds. Phytochemistry and Agriculture. New York: Oxford University Press; 1993: 18-39

[98] Pant N, Garg HS, Madhusudanan KP, Bhakuni DS. Sulfurous compounds from Azadirachta indicia leaves. Fitoterapia 1986; 57: 302

[99] Balandrin MF, Lee SM, Klocke JA. Biologically active volatile organosulfur compounds from seeds of the neem tree, Azadirachta indica (Meliaceae). J Agric Food Chem 1988; 36: 1048-1054

[100] Lavie D, Levy EC, Jain MK. Limonoids of biogenetic interest from Melia azadirachta. Tetrahedron 1971; 27: 3927-3939

[101] Uddin S], Nahar L, Shilpi JA, Shoeb M, Borkowski T, Gibbons S, Middleton M, Byres M, Sarker SD. Gedunin, a limonoid from Xylocarpus granatum, inhibits the growth of $\mathrm{CaCo}-2$ colon cancer cell line in vitro. Phytother Res 2007; 21: 757-761

[102] Gaikwad BR, Mayelvaganan T, Vyas BA, Bhat SV. Nimbocinol and 17 epinimbocinol from the nimbidin fraction of neem oil. Phytochemistry 1990; 29: 3963-3965

[103] Henderson R, McCrindle R, Melera A, Overton KH. Tetranortriterpenoids. IX. The constitution and stereochemistry of salanin. Tetrahedron 1968; 24: 1525-1528

[104] Kubo I, Matsumoto T, Klocke JA. New ecdysis inhibitory limonoid deacetylazadirachtinol isolated from Azadirachta indica (Meliaceae) oil. Tetrahedron 1986; 42: 489-496

[105] Yamasaki BR, Ritland TG, Bornby MA, Klocke JA. Isolation and purification of salanin from neem seeds and its quantitation in neem and chinaberry seeds and leaves. J Chromatography 1988; 447: 277-283

[106] Mitchell M], Smith SL, Johnson S, Morgan ED. Effects of the neem tree compounds azadirachtin, salannin, nimbin, and 6-desacetylnimbin on ecdysone 20-monooxygenase activity. Arch Insect Biochem 1997; 35 : 199-209 
[107] Ekong DEU. Chemistry of the meliacins (limonoids). The structure of nimbolide, a new meliacin from Azadirachta indica. Chem Commun (London) 1967; 16: 808

[108] Rojatkar SR, Bhat VS, Kulkarni MM, Joshi VS, Nagasampagi BA. Tetranortriterpenoids from Azadirachta indica. Phytochemistry 1989; 28: 203-205

[109] Momchilova S, Antonova D, Marekov I, Kuleva L, Damyanova BN, Jhamb G. Fatty acids, triacylglycerols, and sterols in neem oil (Azadirachta indica A. Juss) as determined by a combination of chromatographic and spectral techniques. J Liq Chrom Rel Technol 2007; 30: 111-125

[110] Choi JM, Lee EO, Lee HJ, Kim KH, Ahn KS, Shim BS, Kim NI, Song MC, Baek NI, Kim SH. Identification of campesterol from Chrysanthemum coronarium L. and its antiangiogenic activities. Phytother Res 2007; 21: 954-959

[111] Villaseñor IM, Angelada J, Canlas AP, Echegoyen D. Bioactivity studies on beta-sitosterol and its glucoside. Phytother Res 2002; 16: 417-421

[112] Khanavi M, Gheidarloo R, Sadati N, Ardekani MR, Nabavi SM, Tavajohi S, Ostad SN. Cytotoxicity of fucosterol containing fraction of marine algae against breast and colon carcinoma cell line. Pharmacogn Mag 2012; 8: 60-64

[113] Zhong PU, Zhang Y, Zhong YIN, Jiao XU, Ren JIA, Yang LU, Yang F. Antibacterial activity of 9-octadecanoic acid-hexadecanoic acid-tetrahydrofuran-3,4-diyl ester from neem oil. Agric Sci China 2010; 9: $1236-1240$

[114] Kikuchi T, Ishii K, Noto T, Takahashi A, Tabata K, Suzuki T, Akihisa T. Cytotoxic and apoptosis-inducing activities of limonoids from the seeds of Azadirachta indica (neem). J Nat Prod 2011; 74: 866-870

[115] Kraus W. Biologically active compounds from Meliaceae. Stud Org Chem 1984; 17: 331-345

[116] Siddiqui S, Ghiasuddin, Siddiqui SB, Faizi S. Tetranortriterpenoids and steroidal glycosides from the seeds of Azadirachta indica. Pak J Sci Ind Res 1989; 32: 435

[117] Devakumar C, Mukerjee SK. 4-Epi nimbin, a new meliacin from Azadirachta indica A. Juss. Indian J Chem 1985; 24B: 1105

[118] Bokel M, Cramer R, Gutzeit H, Reeb S, Kraus W. Tetranortriterpenoids related to nimbin and nimbolide from Azadirachta indica A. Juss (Meliaceae). Tetrahedron 1990; 46: 775-782

[119] Cohen E, Ouistad GB, Jefferies PR, Casida JE. Nimbolide is the principal cytotoxic component of neem-seed insecticide preparations. Pestic Sci 1996; 48: 135-140

[120] Priyadarsini RV, Murugan RS, Sripriya P, Karunagaran D, Nagini S. The neem limonoids azadirachtin and nimbolide induce cell cycle arrest and mitochondria-mediated apoptosis in human cervical cancer (HeLa) cells. Free Radic Res 2010; 44: 624-634

[121] Chen J, Chen J, Sun Y, Yan Y, Kong L, Li Y, Qiu M. Cytotoxic triterpenoids from Azadirachta indica. Planta Med 2011; 77: 1844-1847

[122] Elumalai P, Gunadharini DN, Senthilkumar K, Banudevi S, Arunkumar R, Benson CS, Sharmila G, Arunakaran J. Induction of apoptosis in human breast cancer cells by nimbolide through extrinsic and intrinsic pathway. Toxicol Lett 2012; 215: 131-142

[123] Kigodi PG, Blaskó G, Thebtaranonth Y, Pezzuto JM, Cordell GA. Spectroscopic and biological investigation of nimbolide and 28-deoxonimbolide from Azadirachta indica. J Nat Prod 1989; 52: 1246-1251

[124] Ochi M, Kotsuki H, Kataoka T, Tada T, Tokoroyama T. Limonoids from Melia azedarach Linn Var. Japonica makino III. The structure of ochinal and ochinin. Chem Lett 1978; 4: 331

[125] Butterworth $\mathrm{JH}$, Morgan ED. Isolation of a substance that supresses feeding in locust. J Chem Soc Chem Commun 1968: 23-24

[126] Morgan ED, Thornton MD. Azadirachtin in the fruit of Melia azedarach. Phytochemistry 1973; 12: 391-392
[127] Kraus W. Constituents of neem and related species. A revised structure of azadirachtin. Stud Org Chem (Amsterdam) 1986; 26: 237-256

[128] Rembold H, Forster H, Sonnebichller J. Structure of azadirachtin B. Z Naturforch 1989; 42 C: 4-6

[129] Rojatkar SR, Nagasampagi BA. a-acetoxy-1a-hydroxyazadirachtol, a new constituent from Azadirachta indica (A. Juss.) seeds. Indian J Chem 1995; 34B: 1016-1018

[130] Rojatkar SR, Nagasampagi BA. 11 $\alpha$-hydroxy-12-norazadirachtin from Azadirachta indica (A. Juss.). Nat Prod Lett 1994; 5: 6976

[131] Govindachari TR, Sandhya G, Ganeshraj SP. Isolation of novel azadirachtins $\mathrm{H}$ and $\mathrm{I}$ by high performance liquid chromatography. Chromatographia 1991; 31: 303-305

[132] Govindachari TR, Gopalakrishnan G, Suresh G. Isolation of various azadirachtins from neem oil by preparative high performance liquid chromatography. J Liq Chrom Rel Technol 1996; 19: 1729-1733

[133] Govindachari TR, Sandhya G, Ganeshraj SP. Azadirachtin H and I: two new tetranortriterpenoids from Azadirachta indica. J Nat Prod 1992; 55: 596-601

[134] Garg HS, Bhakuni DS. Salannolide, a meliacin from Azadirachta indica. Phytochemistry 1984; 23: 2383-2385

[135] Kraus W, Baumann S, Bokel M, Cramer R, Grimminger W, Hendlemeir R, Keil E, Keller U, Klenk A, Klingele MPH, Schwinger M. Insect feeding and development controlling constituents of Meliaceae. In: Proc Ist Princess Chulabhorn Sci Cong, Ist Cong on Nat Prod Bangkok, vol 2; 1987: 554

[136] Kumar CS, Srinivas M, Yakkundi S. Limonoids from the seeds of Azadirachta indica. Phytochemistry 1996; 43: 451-455

[137] Bilton JN, Broughton HB, Jones PS, Ley SV, Rzepa HS, Sheppard RN Slawin AMZ, Williams DJ, Lidert Z, Morgan ED. An x-ray crystallographic, mass spectroscopic, and NMR study of the limonoid insect antifeedant azadirachtin and related derivatives. Tetrahedron 1987; 43: 2805-2815

[138] Govindachari TR, Sandhya G, Ganeshraj SP. Structure of azadirachtin K, a new tetranortriterpenoid from Azadirachta indica. Indian J Chem 1992; 31 B: 295-298

[139] Ramji N, Venkatakrishnan K, Madyastha KM. 11-epi-azadirachtin H from Azadirachta indica. Phytochemistry 1996; 42: 561

[140] Rojatkar SR, Nagasampagi BA. 1-tigloyl-3-acetyl-11-hydroxy-4ß-methylmeliacarpin from Azadirachta indica. Phytochemistry 1992; 32: 213214

[141] Ramji N, Venkatakrishnan K, Madyastha KM. 11-epi-azadirachtin D: an epimeric azadirachtin analogue from Azadirachta indica. Phytochemistry 1998; 49: 265-267

[142] Mitra CR, Garg HS, Pandey GN. Constituents of Melia indica-II nimbidic acid and nimbidinin. Tetrahedron Lett 1970; 11: 2761-2764

[143] Mitra CR, Garg HS, Pandey GN. Identification of nimbidic and nimbidinin from Azadirachta indica. Phytochemistry 1970; 10: 857

[144] Akhila A, Srivastava M, Rani K. Production of radioactive azadirachtin in the seed kernels of Azadirachta indica. J Nat Prod 1998; 11: 107-110

[145] Luo X, Ma Y, Wu S, Wu D. Two novel azadirachtin derivatives from Azadirachta indica. J Nat Prod 1999; 62: 1022-1024

[146] Klenk A, Bokel M, Kraus W. 3-Tigloylazadirachtol (tigloyl-2-methylcrotonoyl), an insect growth regulating constituent of Azadirachta indica. J Chem Soc Chem Commun 1986: 523-524

[147] Bilton JN, Jones PS, Ley SV, Robinson NG, Sheppard RN. Chemistry of insect antifeedants from Azadirachta indica. Part I: Conversion from the azadirachtin to the azadirachtinin skeletons. Tetrahedron Lett 1988; 29: 1849-1852

[148] Govindachari TR, Gopalakrishnan G, Suresh G. Purification of azadirachtin-B (3-tigloylazadirachtol) by preparative high performance liquid chromatography, using the recycling mode. I Liq Chrom Rel Technol 1997; 20: 1633-1636 
[149] Grimalt S, Thompson DG, Coppens M, Chartrand DT, Shorney T. Meating J, Scarr T. Analytical study of azadirachtin and 3-tigloylazadirachtol residues in foliage and phloem of hardwood tree species by liquid chromatography-electrospray mass spectrometry. J Agric Food Chem 2011; 59: 8070-8077

[150] Govindachari TR, Gopalakrishnan G. 13,14-desepoxy-azadirachtin-A, a tetranortriterpenoid from Azadirachta indica. Phytochemistry 1997; 45: 397-399

[151] Kraus W, Klenk A, Bokel M, Vogler B. Tetranortriterpenoid lactams with insect antifeeding activity from Azadirachta indica A. Juss (Meliaceae). Liebig Ann Chem 1987; 4: 337-340

[152] Siddiqui S, Mahmood T, Siddiqui BS, Faizi S. Triterpenoids from kernel of Azadirachta indica. Proc Pak Acad Sci 1990; 27: 333-348

[153] Ghiasuddin. Chemical studies on the constituents of Azadirachta indica A. Juss. [Ph.D. Dissertation]. Karachi: University of Karachi; 1993

[154] Wang HW, Liu JQ, Chen JX, Yang YF, Yan YX, Li ZR, Qiu MH. New triterpenoids from the kernels of Azadirachta indica. Nat Prod Bioprospect 2013; 3: 33-37

[155] Mc Inerney BV, Taylor WC. The Xenocoumarins and related biologically active Dihydroisocoumarins. In: Rahman A, ed. Studies in natural Products Chemistry, Vol 15. Elsevier: Amsterdam; 1995: 381-422

[156] Kurosaki F, Nishi A. Isolation and antimicrobial activity of the phytoalexin 6-methoxymellein from cultured carrot cells. Phytochemistry 1983; 22: 669-672

[157] Borris RP, Cordell GA, Farnsworth NR. Isofraxidin, a cytotoxic coumarin from Micrandra elata (Euphorbiaceae). J Nat Prod 1980; 43: 641-643

[158] Siddiqui S, Mahmood T, Siddiqui BS, Faizi S. Isonimolide and isonimbolide, two new tetranortriterpenoids from the twigs of Azadirachta indica A. Juss (Meliaceae). Heterocycles 1987; 26: 1827-1833

[159] Siddiqui S, Faizi S, Mahmood T, Siddiqui BS. Margosinolide and isomargosinolide, two new tetranortriteprenoids from Azadirachta indica A. Juss (Meliaceae). Tetrahedron 1986; 42: 4849-4856

[160] Siddiqui S, Mahmood T, Siddiqui BS, Faizi S. Two new tetranortriterpenoids from Azadirachta indica. J Nat Prod 1986; 49: 1068-1073

[161] Ara I, Siddiqui BS, Faizi S, Siddiqui S. Three new diterpenoids from the stem bark of Azadirachta indica. J Nat Prod 1990; 53: 816-820

[162] Ara I, Siddiqui BS, Faizi S, Siddiqui S. Structurally novel diterpenoid constituents from the stem bark of Azadirachta indica. J Chem Soc Perkin Trans I 1989: 343-345

[163] Ara I, Siddiqui BS, Faizi S, Siddiqui S. Tricyclic diterpenes from the stem bark of Azadirachta indica. Planta Med 1990; 56: 84-86

[164] Ara I, Siddiqui BS, Faizi S, Siddiqui S. Tricyclic diterpenoids from the stem bark of Azadirachta indica. J Nat Prod 1988; 51: 1054-1061

[165] Ara I, Siddiqui BS, Faizi S, Siddiqui S. Terpenoids from the stem bark of Azadirachta indica. Phytochemistry 1988; 27: 1801-1804

[166] Siddiqui S, Ara I, Faizi S, Mahmood T, Siddiqui BS. Phenolic tricyclic diterpenoids from the bark of Azadirachta indica. Phytochemistry 1988; 27: 3903-3907

[167] Ara I, Siddiqui BS, Faizi S, Siddiqui S. Diterpenoids from the stem bark of Azadirachta indica. Phytochemistry 1989; 28: 1177-1180

[168] Sengupta P, Choudhuri SN, Khastgir HN. Investigations of the trunk bark of Melia azadirachta. Linn Chem and Ind (London) 1958; 1958: 861-862

[169] Sengupta P, Chowdhury SN, Khastagir HN. Terpenoids and related compounds - I. Constituents of the trunk bark of Melia azadirachta Linn and the structure of the ketophenol, nimbiol. Tetrahedron 1960; 10: $45-54$

[170] Sengupta P, Sengupta SK, Khastgir HN. Terpenoids and related compounds - II Investigations on the structure of nimbin. Tetrahedron 1960; 11: 67-77

[171] Choudhuri SN, Khastgir HN, Sengupta P. Investigation on the structure of Nimbiol. Chem and Ind (London) 1959; 20: 634-635
[172] Chao KP, Hua KF, Hsu HY, Su YC, Chang ST. Anti-inflammatory activity of sugiol, a diterpene isolated from Calocedrus formosana bark. Planta Med 2005; 71: 300-305

[173] Ara I, Siddiqui BS, Faizi S, Siddiqui S. Margosinone and margosinolone, two new polyacetate derivatives from Azadirachta indica. Fitoterapia 1989; 60: 519-523

[174] Banerji R, Misra G, Nigam SK. Identification of 24-methylene lophenol from heartwood of Azadirachta indica. Phytochemistry 1987; 26: 2644-2645

[175] Ekong DEU, Fakunle CO, Fasina AK, Okogun J. The meliacins (limonoids). Nimbolin A and B, two new meliacin cinnamates from Azadirachta indica L. and Melia azedarach L. J Chem Soc D, Chem Commun 1969; 20: 1166-1167

[176] Nakatani M. Limonoids from Meliaceae and their biological Activities. In: Tringali C, ed. Bioactive Compounds from Natural Sources - Isolation, Characterisation and biological Properties. Boca Raton: Taylor and Francis; 2000: 527-554

[177] Van der Nat JM, van der Sluis WG, 't Hart LA, Van Dijk H, de Silva KT, Labadie RP. Activity-guided isolation and identification of Azadirachto indica bark extract constituents which specifically inhibit chemiluminescence production by activated human polymorphonuclear leukocytes. Planta Med 1991; 57: 65-68

[178] Yang B, Kotani A, Arai K, Kusu F. Estimation of the antioxidant activities of flavonoids from their oxidation potentials. Anal Sci 2001; 17: 599604

[179] Miyazawa M, Shimamura H, Nakamura S. Antimutagenic activity of isofraxinellone from Dictamnus dasycarpus. J Agric Food Chem 1995; 43: $1428-1431$

[180] Banerji B, Nigam SK. Wood constituents of Meliaceae: a review. Fitoterapia 1984; 55: 3-36

[181] Simmonds MSJ, Jarvis AP, Johnson S, Jones GR, Morgan ED. Comparison of anti-feedant and insecticidal activity of nimbin and salannin photooxidation products with neem (Azadirachta indica) limonoids. Pest Manag Sci 2004; 60: 459-464

[182] Ara I. Studies in the chemical constituents of Azadirachta indica (neem) [Ph.D. Dissertation]. Karachi: University of Karachi; 1989

[183] Ara I, Siddiqui BS, Faizi S, Siddiqui S. Isolation and structure elucidation of the triterpene azadirinin from the roots of Azadirachta indica. Fitoterapia 1992; 63: 118

[184] Ara I, Siddiqui BS, Faizi S, Siddiqui S. Tricyclic diterpenoids from root bark of Azadirachta indica. Phytochemistry 1990; 29: 911-914

[185] Majumder PL, Maiti DC, Kraus W, Bokel M. Nimbidiol, a modified diterpenoid of the root-bark of Azadirachta indica. Phytochemistry 1987; 26: 3021-3023

[186] Ekong DEU, Olagbemi EO, Spiff Al. Cycloeucalenol and 24-methylenecycloartanol in wood oils from the family Meliaceae. Chem Ind (London) 1968; 1968: 1808

[187] Kiribuchi M, Miura K, Tokuda S, Kaneda T. Hypocholesterolemic effect of triterpene alcohols with soysterol on plasma cholesterol in rats. J Nutr Sci Vitaminol (Tokyo) 1983; 29: 35-43

[188] Mukherjee S, Srivastava HC. Structure of neem gum. J Am Chem Soc 1955; 77: 422

[189] Anderson DMW, Hendrie A. The proteinaceous gum polysacheride from Azadirachta indica. Carbohyd Res 1979; 20: 259-268

[190] Lakshmi SU, Pattabiraman TN. Studies on the plant gum: Part I. Identification of nitrogenous compounds in neem (Azadirachta indica) gum and isolation of D-glucosamin. Indian J Biochem 1967; 4: 183

[191] Bajpai KS, Chandrasekharan V, Mukherjee S, Srivastava AN. Isolation of pure aldobiouronie and aldotriouronic acid from the roots of Azadirachta indica. Fitoterapia 1970; 63: 118

[192] Lavie D, Jain MK. Tetranortriterpenoids from Melia azadirachta L. Chem Commun 1967: 278-280 
[193] Akhila A, Rani K. Chemistry of the neem tree (Azadirachta indica A. Juss.). Fortschr Chem Org Naturst 1999; 78: 47-149

[194] Madhusudanan KP, Chaturvedi R, Garg HS, Bhakuni DS. Negative ion mass spectra of tetranortriterpenoids isolated from neem (Azadirachta indica A. Juss.). Indian J Chem Sect B 1984; 23: 1082

[195] Gossé B, Amissa AA, Adjé FA, Niamké FB, Ollivier D, Ito Y. Analysis of components of neem (Azadirachta indica) oil by diverse chromatographic techniques. J Liq Chrom Rel Technol 2005; 28: 14, 2225-2233

[196] Rizvi SH, Kapil RS, Shoeb A. Alkaloids and coumarins of Casimiroa edulis. J Nat Prod 1985; 48: 146

[197] Mitra C. On the constitution of nimbin. J Sci Ind Res (India) 1957; 16 B: 477

[198] Sadeghian MM, Mortazaienezhad F. Investigation of compounds from Azadirachta indica (neem). Asian J Plant Sci 2007; 6: 444-445

[199] Lavie D, Jain MK, Shpan-Gabrielith SR. A locust phagorepellent from two melia species. Chem Commun 1967: 910-911

[200] Garg HS, Bhakuni DS. An isoprenylated flavanone from leaves of Azadirachta indica. Phytochemistry 1984; 23: 2115-2118

[201] Panda S, Kar A. Evaluation of the antithyroid, antioxidative and antihyperglycemic activity of scopoletin from Aegle marmelos leaves in hyperthyroid rats. Phytother Res 2006; 20: 1103-1105

[202] Rukmini C. Chemical and nutritional evaluation of neem oil. Food Chem 1987; 26: 119-124

[203] Devakumar C, Mukerjee SK. Chemistry of neem bitter principles. IARI Research Bulletin 1983; 40: 1-17

[204] Yamasaki RB, Klocke JA. Structure-bioactivity relationships of azadirachtin, a potent insect control agent. J Agric Food Chem 1987; 35: 467-471

[205] Sankaram AVB, Murthy MM, Bhaskariah K, Subramany M, Sultana N, Sharma HC, Leischner K. 16th International Symposium on the Chemistry of Natural Products. IUPAC, Japan: Kyoto, Abs. No 1539; 1988: 41

[206] Teng BS, Lu YH, Wang ZT, Tao XY, Wei DZ. In vitro anti-tumor activity of isorhamnetin isolated from Hippophae rhamnoides L. against BEL7402 cells. Pharmacol Res 2006; 54: 186-194

[207] Goel RK, Pandey VB, Dwivedi SP, Rao YV. Antiinflammatory and antiulcer effects of kaempferol, a flavone, isolated from Rhamnus procumbens. Indian J Exp Biol 1988; 26: 121-124

[208] Pekkarinen SS, Heinonen IM, Hopia AI. Flavonoids quercetin, myricetin, kaemferol and (+)-catechin as antioxidants in methyl linoleate. J Sci Food Agric 1999; 79: 499-506

[209] Kaur G, Sarwar Alam M, Athar M. Nimbidin suppresses functions of macrophages and neutrophils: relevance to its antiinflammatory mechanisms. Phytother Res 2004; 18: 419-424

[210] Mosaddek ASM, Rashid MMU. A comparative study of the anti-inflammatory effect of aqueous extract of neem leaf and dexamethasone. Bangladesh J Pharmacol 2008; 3: 44-47

[211] Mahabub-Uz-Zaman M, Ahmed NU, Akter R, Ahmed K, Aziz MSI, Ahmed MS. Studies on anti-inflammatory, antinociceptive and antipyretic activities of ethanol extract of Azadirachta indica leaves. Bangladesh J Sci Ind Res 2009; 44: 199-206

[212] Upadhyay SN, Dhawan S, Garg S, Talwar GP. Immunomodulatory effects of neem (Azadirachta indica) oil. Int J Immunopharmacol 1992; 14: 1187-1193

[213] SaiRam M, Sharma SK, Ilavazhagan G, Kumar D, Selvamurthy W. Immunomodulatory effects of NIM-76, a volatile fraction from neem oil. J Ethnopharmacol 1997; 55: 133-139

[214] Kumar A, Rao AR, Kimura H. Radiosensitizing effects of neem (Azadirachta indica) oil. Phytother Res 2002; 16: 74-77

[215] Bopanna KN, Kannan J, Sushma G, Balaraman R, Rathod SP. Antidiabetic and antihyperlipaemic effects of neem seed kernel powder on alloxan diabetic rabbits. Indian J Pharmacol 1997; 29: 162-167
[216] Bajaj S, Srinivasan BP. Investigations into the anti-diabetic activity of Azadirachta indica. Indian J Pharmacol 1999; 31: 138-141

[217] Halim EM. Lowering of blood sugar by water extract of Azadirachta indica and Abroma augusta in diabetes rats. Indian J Exp Biol 2003; 41: 636-640

[218] Ebong PE, Atangwho I], Eyong EU, Egbung GE. The antidiabetic efficacy of combined extracts from two continental plants: Azadirachta indica (A. Juss) (neem) and Vernonia amygdalina (Del.) (african bitter leaf). Am J Biochem Biotechnol 2008; 4: 239-244

[219] Bisht S, Sisodia SS. Anti-hyperglycemic and antidyslipidemic potentia of Azadirachta indica leaf extract in STZ-induced diabetes mellitus. J Pharma Sci Res 2010; 2: 622-627

[220] Dholi SK, Raparla R, Mankala SK, Nagappan K. In vivo antidiabetic evaluation of neem leaf extract in alloxan induced rats. J App Pharma Sci 2011; 1: 100-105

[221] Garg GP, Nigam SK, Ogle CW. The gastric antiulcer effects of the leaves of the neem tree. Planta Med 1993; 59: 215-217

[222] Chattopadhyay I, Nandi B, Chatterjee R, Biswas K, Bandyopadhyay U, Banerjee RK. Mechanism of antiulcer effect of neem (Azadirachta indica) leaf extract: effect on $\mathrm{H}+-\mathrm{K}+-$ ATPase, oxidative damage and apoptosis. Inflammopharmacology 2004; 12: 153-176

[223] Upadhyay SN, Kaushic C, Talwar GP. Antifertility effects of neem (Azadirachta indica) oil by single intrauterine administration: a novel method for contraception. Proc Biol Sci 1990; 242: 175-179

[224] Upadhyay SN, Dhawan S, Talwar GP. Antifertility effects of neem (Azadirachta indica) oil in male rats by single intra-vas administration: an alternate approach to vasectomy. J Androl 1993; 14: 275-281

[225] Sharma SK, SaiRam M, Ilavazhagan G, Devendra K, Shivaji SS Selvamurthy W. Mechanism of action of NIM-76: a novel vaginal contraceptive from neem oil. Contraception 1996; 54: 373-378

[226] Riar SS, Devakumar C, Sawhney RC, llavazhagan G, Bardhan J, Kain AK, Thomas P, Singh R, Singh B, Parshad R. Antifertility activity of volatile fraction of neem oil. Contraception 1991; 44: 319-326

[227] Khillare B, Shrivastav TG. Spermicidal activity of Azadirachta indica (neem) leaf extract. Contraception 2003; 68: 225-229

[228] Khalid SA, Duddeck H, Gonzales-Sierra M. Isolation and characterization of an antimalarial agent of the neem tree Azadirachta indica. J Nat Prod 1989; 52: 922-927

[229] Vasanth S, Gopal RH, Rao RH, Rao RB. Plant antimalarial agents. J Sc Ind Res 1990; 49: 68-77

[230] Schwikkard S, van Heerden FR. Antimalarial activity of plant metabolites. Nat Prod Rep 2002; 19: 675-692

[231] Lucantoni L, Yerbanga RS, Lupidi G, Pasqualini L, Esposito F, Habluetzel A. Transmission blocking activity of a standardized neem (Azadirachta indica) seed extract on the rodent malaria parasite Plasmodium berghei in its vector Anopheles stephensi. Malaria J 2010; 9: 66

[232] Udeinya IJ, Mbah AU, Chijioke CP, Shu EN. An anti-malarial extract from neem leaves is antiretroviral. Trans Royal Soc Trop Med Hyg 2004; 98: 435-437

[233] Mbah AU, Udeinya IJ, Shu EN, Chijioke CP, Nubila T, Udeinya F, Muobuike A, Mmuobieri A, Obioma MS. Fractionated neem leaf extract is safe and increases CD4+ cell levels in HIV/AIDS patients. Am J Ther 2007; 14: 369-374

[234] Khan MI, Shah NH. Antifungal activity of leaf extract of neem on seed mycoflora of wheat. Bioved 1992; 3: 209-210

[235] Suresh G, Narasimhan NS, Masilamani S, Partho PD, Gopalakrishnan G. Antifungal fractions and compounds from uncrushed green leaves of Azadirachta indica. Phytoparasitica 1997; 25: 33-39

[236] Govindachari TR, Suresh G, Geetha G, Banumathy B, Masilamani S. Identification of antifungal compounds from the seed oil of Azadirachta indica. Phytopathology 1998; 26: 109-116 
[237] Natarajan V, Venugopal PV, Menon T. Effect of Azadirachta indica (neem) on the growth pattern of dermatophytes. Indian J Med Microbiol 2003; 21: 98-101

[238] Wang J, Li J, Cao J, Jiang W. Antifungal activities of neem (Azadirachta indica) seed kernel extracts on postharvest diseases in fruits. Afr J Microbiol Res 2010; 4: 1100-1104

[239] Das BK, Mukherjee SC, Sahu BB, Murjani G. Neem (Azadirachta indica) extract as an antibacterial agent against fish pathogenic bacteria. Indian J Exp Biol 1999; 37: 1097-1100

[240] SaiRam M, Ilavazhagan G, Sharma SK, Dhanraj SA, Suresh B, Parida MM, Jana AM, Devendra K, Selvamurthy W. Anti-microbial activity of a new vaginal contraceptive NIM-76 from neem oil (Azadirachta indica). J Ethnopharmacol 2000; 71: 377-382

[241] Coventry E, Allan EJ. Microbiological and chemical analysis of neem (Azadirachta indica) extracts: new data on antimicrobial activity. Phytoparasitica 2001 ; 29 : 441-450

[242] Vanka A, Tandon S, Rao SR, Udupa N, Ramkumar P. The effect of indigenous neem Azadirachta indica mouth wash on Streptococcus mutans and Lactobacilli growth. Indian J Dent Res 2001; 12: 133-144

[243] Pai MR, Acharya LD, Udupa U. Evaluation of antiplaque activity of Azadirachta indica leaf extract gel-a 6-week clinical study. J Ethnopharmacol 2004; 90: 99-103

[244] Joshi M, Ali SW, Rajendran S. Antibacterial finishing of polyester/cotton blend fabrics using neem (Azadirachta indica): a natural bioactive agent. J Appl Polym Sci 2007; 106: 793-800

[245] Jahan T, Begum ZA, Sultana S. Effect of neem oil on some pathogenic bacteria. Bangladesh J Pharmacol 2007; 2: 71-72

[246] Matthews RL, Templeton MR, Tripathi SK, Bhattarai K. Disinfection of waterborne coliform bacteria by neem oil. Environ Eng Sci 2009; 26: 1435-1441

[247] Aslam F, Rehman K, Asghar M, Sarwar M. Antibacterial activity of various phytoconstituents of neem. Pak J Agri Sci 2009; 46: 209-213

[248] Zhang YQ, Xu J, Yin ZQ, Jia RY, Lu Y, Yang F, Du YH, Zou P, Lv C, Hu TX, Liu SL, Shu G, Yi G. Isolation and identification of the antibacterial active compound from petroleum ether extract of neem oil. Fitoterapia 2010; 81: 747-750

[249] Maragathavalli S, Brindha S, Kaviyarasi NS, Annadurai B, Gangwar SK. Antimicrobial activity in leaf extract of neem (Azadirachta indica Linn.). Int J Sci Nature 2012; 3: 110-113

[250] Lowery DT, Eastwell KC, Smirle M]. Neem seed oil inhibits aphid transmission of potato virus Y to pepper. Ann Appl Biol 1997; 130: 217-225

[251] Badman L, Joshi SP, Bedekar SS. In vitro antiviral activity of neem (Azadirachta indica, A. Juss) leaf extract against coxsachieviruses. J Commun Dis 1999; 31: 79-90

[252] Parida MM, Upadhyay C, Pandya G, Jana AM. Inhibitory potential of neem (Azadirachta indica Juss) leaves on dengue virus type-2 replication. J Ethnopharmacol 2002; 79: 273-278

[253] Tiwari V, Darmani NA, Yue BY, Shukla D. In vitro antiviral activity of neem (Azardirachta indica L.) bark extract against herpes simplex virus type-1 infection. Phytother Res 2010; 24: 1132-1140

[254] Amer H, Helmy WA, Taie HAA. In vitro antitumor and antiviral activities of seeds and leaves neem (Azadirachta indica) extracts. Int J Acad Res 2010; 2: 47-51

[255] Nanduria S, Thunuguntlaa SSR, Nyavanandia VK, Kasua S, Kumara MP, Rama PS, Rajagopalb S, Kumarb RA, Deevib DS, Rajagopalanb R, Venkateswarlua A. Biological investigation and structure-activity relationship studies on azadirone from Azadirachta indica A. Juss. Bioorg Med Chem Lett 2003; 13: 4111-4115

[256] Balasenthil S, Arivazhagan S, Ramachandran CR, Ramachandran V, Nagini S. Chemopreventive potential of neem (Azadirachta indica) on 7,12-dimethylbenz[a]anthracene (DMBA) induced hamster buccal pouch carcinogenesis. J Ethnopharmacol 1999; 67: 189-195
[257] Subapriya R, Kumaraguruparan R, Chandramohan KV, Nagini S. Chemoprotective effects of ethanolic extract of neem leaf against MNNG-induced oxidative stress. Pharmazie 2003; 58: 512-517

[258] Dasgupta T, Banerjee S, Yadava PK, Rao AR. Chemopreventive potential of Azadirachta indica (neem) leaf extract in murine carcinogenesis model systems. J Ethnopharmacol 2004; 92: 23-36

[259] Subapriya R, Bhuvaneswari V, Nagini S. Ethanolic neem (Azadirachto indica) leaf extract induces apoptosis in the hamster buccal pouch carcinogenesis model by modulation of $\mathrm{Bcl}-2$, Bim, caspase 8 and caspase 3. Asian Pac J Cancer Prev 2005; 6: 515-520

[260] Arakaki J, Suzui M, Morioka T, Kinjo T, Kaneshiro T, Inamine M, Sunagawa N, Nishimaki T, Yoshimi N. Antioxidative and modifying effects of a tropical plant Azadirachta indica (neem) on azoxymethane-induced preneoplastic lesions in the rat colon. Asian Pac J Cancer Prev 2006; 7: 467-471

[261] Roy MK, Kobori M, Takenaka M, Nakahara K, Shinmoto H, Isobe S, Tsushida T. Antiproliferative effect on human cancer cell lines after treatment with nimbolide extracted from an edible part of the neem tree (Azadirachta indica). Phytother Res 2007; 21: 245-250

[262] Vijayan V, Meshram GP. Genotoxicity assessment of NIM-76 and its formulation (pessary) in an in vitro Ames Salmonella/microsome assay and in vivo mouse bone marrow micronucleus test. Drug Chem Toxicol 2013; 36: 430-434

[263] Mallick A, Barik S, Goswami KK, Banerjee S, Ghosh S, Sarkar K, Bose A Baral R. Neem leaf glycoprotein activates CD8(+) T cells to promote therapeutic anti-tumor immunity inhibiting the growth of mouse sarcoma. PLoS One 2013; 8: e47434. doi:10.1371/journal.pone. 0047434

[264] Mallick A, Ghosh S, Banerjee S, Majumder S, Das A, Mondal B, Barik S, Goswami KK, Pal S, Laskar S, Sarkar K, Bose A, Baral R. Neem leaf glycoprotein is nontoxic to physiological functions of Swiss mice and Sprague-Dawley rats: histological, biochemical and immunological perspectives. Int Immunopharmacol 2013; 15: 73-83

[265] Srivastava P, Yadav N, Lella R, Schneider A, Jones A, Marlowe T, Lovett G, O’Loughlin K, Minderman H, Gogada R, Chandra D. Neem oil limonoids induces p53-independent apoptosis and autophagy. Carcinogenesis 2012; 33: 2199-2207

[266] Kale BP, Kothekar MA, Tayade HP, Jaju JB, Mateenuddin M. Effect of aqueous extract of Azadirachta indica leaves on hepatotoxicity induced by antitubercular drugs in rats. Indian J Pharmacol 2003; 35: 177-180

[267] Chattopadhyay RR, Bandyopadhyay M. Possible mechanism of hepatoprotective activity of Azadirachta indica leaf extract against paracetamol-induced hepatic damage in rats: Part III. Indian J Pharmacol 2005; 37: 184-185

[268] Mohamed ET, Mahran HA, Mahmoud MS. Hepato ameliorative effect of Azadirachta indica leaves extract against mercuric chloride environmental pollution. J Am Sci 2010; 6: 735-751

[269] Sithisarn P, Supabphol R, Gritsanapan W. Comparison of free radica scavenging activity of Siamese neem tree (Azadirachta indica A. Juss var. siamensis Valeton) leaf extracts prepared by different methods of extraction. Med Princ Pract 2006; 15: 219-222

[270] Ghimeray AK, Jin CW, Ghimire BK, Cho DH. Antioxidant activity and quantitative estimation of azadirachtin and nimbin in Azadirachta Indica A. Juss grown in foothills of Nepal. Afr J Biotechnol 2009; 8: 30843091

[271] Jaiswal AK, Bhattacharya SK, Acharya SB. Anxiolytic activity of Azadirachta indica leaf extract in rats. Indian J Exp Biol 1994; 32: 489-491

[272] Samad N, Parveen T, Haider S, Haleem DJ. Attenuation of stress-induced behavioural deficits by Azadirachta indica (neem): role of serotonin. Pak J Bot 2006; 38: 131-138

[273] Hiremath SB, Srinivas LD. A study on anxiolytic activity of Azadirachto indica leaves in wistar albino rats. Int J Adv Pharma Res 2011; 2: 563 568 
[274] Singh K, Singh A, Singh DK. Molluscicidal activity of neem (Azadirachto indica A. Juss). J Ethnopharmacol 1996; 52: 35-40

[275] Ebenso IE. Molluscicidal effects of neem (Azadirachta indica) extracts on edible tropical land snails. Pest Manag Sci 2004; 60: 178-182

[276] Rahuman AA, Gopalakrishnan G, Venkatesan P, Geetha K. Isolation and identification of mosquito larvicidal compound from Abutilon indicum (Linn.) Sweet. Parasitol Res 2008; 102: 981-988

[277] Djenontin TS, Wottoa VD, Avlessia F, Lozanob P, Sohounhlouéa DKC, Piochb D. Composition of Azadirachta indica and Carapa procera (Meliaceae) seed oils and cakes obtained after oil extraction. Ind Crops Prod 2012; 38: 39-45

[278] Dua VK, Pandey AC, Raghavendra K, Gupta A, Sharma T, Dash AP. Larvicidal activity of neem oil (Azadirachta indica) formulation against mosquitoes. Malaria J 2009; 8: 124

[279] Ghaffar FA, Semmler M. Efficacy of neem seed extract shampoo on head lice of naturally infected humans in Egypt. Parasitol Res 2007; 100: 329-332

[280] Du YH, Jia RY, Yin ZQ, Pu ZH, Chen J, Yang F, Zhang YQ, Lu Y. Acaricidal activity of extracts of neem (Azadirachta indica) oil against the larvae of the rabbit mite Sarcoptes scabiei var. cuniculi in vitro. Vet Parasitol 2008; 157: 144-148

[281] Deng Y, Shi D, Yin Z, Guo J, Jia R, Xu J, Song X, Lv C, Fan Q, Liang X, Shi F, Ye G, Zhang W. Acaricidal activity of petroleum ether extract of neem (Azadirachta indica) oil and its four fractions separated by column chromatography against Sarcoptes scabiei var. cuniculi larvae in vitro. Exp Parasitol 2012; 130: 475-477

[282] Du YH, Li JL, Jia RY, Yin ZQ, Li XT, Lv C, Ye G, Zhang L, Zhang YQ. Acaricidal activity of four fractions and octadecanoic acid-tetrahydrofuran-3,4-diyl ester isolated from chloroform extracts of neem (Azadirachta indica) oil against Sarcoptes scabiei var. cuniculi larvae in vitro. Vet Parasitol 2009; 163: 175-178

[283] Xu J, Fan Q], Yin ZQ, Li XT, Du YH, Jia RY, Wang KY, Lv C, Ye G, Geng Y, Su G, Zhao L, Hu TX, Shi F, Zhang L, Wu CL, Tao C, Zhang YX, Shi DX. The preparation of neem oil microemulsion (Azadirachta indica) and the comparison of acaricidal time between neem oil microemulsion and other formulations in vitro. Vet Parasitol 2010; 169: 399-403

[284] Mishra V, Parveen N, Singhal KC, Khan NU. Antifilarial activity of Azadirachta indica on cattle filarial parasite Setaria cervi. Fitoterapia 2005; 76: 54-61

[285] Zanno P, Miura I, Nakanishi K, Elder DL. Letter: structure of the insect phagorepellent azadirachtin. Application of PRFT/CWD carbon-13 nuclear magnetic resonance. J Am Chem Soc 1975; 97: 1975-1977

[286] Jauch J. Total synthesis of azadirachtin - finally completed after 22 years. Angew Chem Int Ed Engl 2008; 47: 34-37

[287] Ley SV, Abad-Somovilla A, Anderson JC, Ayats C, Bänteli R, Beckmann E, Boyer A, Brasca MG, Brice A, Broughton HB, Burke BJ, Cleator E, Craig D, Denholm AA, Denton RM, Durand-Reville T, Gobbi LB, Göbel M, Gray BL, Grossmann RB, Gutteridge CE, Hahn N, Harding SL, Jennens DC, Jennens L, Lovell PJ, Lovell HJ, de la Puente ML, Kolb HC, Koot WJ, Maslen SL, McCusker CF, Mattes A, Pape AR, Pinto A, Santafianos D, Scott JS, Smith SC, Somers AQ, Spilling CD, Stelzer F, Toogood PL, Turner RM, Veitch GE, Wood A, Zumbrunn C. The synthesis of azadirachtin: a potent insect antifeedant. Chemistry 2008; 14: 1068310704

[288] Veitch GE, Beckmann E, Burke B], Boyer A, Maslen SL, Ley SV. Synthesis of azadirachtin: a long but successful journey. Angew Chem Int Ed Engl 2007; 46: 7629-7632

[289] Prakash G, Bhojwani SS, Srivastava AK. Production of azadirachtin from plant tissue culture-state of the art and future prospects. Biotechnol Bioprocess Eng 2002; 7: 185-193

[290] Allan EJ, Eeswara JP, Jarvis AP, Mordue (Luntz) AJ, Morgan ED, Stuchbury T. Induction of hairy root cultures of Azadirachta indica A. Juss. and their production of azadirachtin and other important insect bioactive metabolites. Plant Cell Rep 2002; 21: 374-379
[291] Satdive RK, Fulzele DP, Eapen S. Enhanced production of azadirachtin by hairy root cultures of Azadirachta indica A. Juss by elicitation and media optimization. J Biotechnol 2007; 128: 281-289

[292] Rafiq M, Dahot MU. Callus and azadirachtin related limonoids production through in vitro culture of neem (Azadirachta indica A. Juss). Afr J Biotechnol 2010; 9: 449-453

[293] Srivastava P, Chaturvedi R. Increased production of azadirachtin from an improved method of androgenic cultures of a medicinal tree Azadirachta indica A. Juss. Plant Signal Behav 2011; 6: 974-981

[294] Kusari S, Verma VC, Lamshoeft M, Spiteller M. An endophytic fungus from Azadirachta indica A. Juss. that produces azadirachtin. World J Microbiol Biotechnol 2012; 28: 1287-1294

[295] Hawksworth DL. The variety of fungal-algal symbioses, their evolutionary significance, and the nature of lichens. Bot J Linn Soc 1988; 96: 3 20

[296] Li WC, Zhou J, Guo SY, Guo LD. Endophytic fungi associated with lichens in Baihua mountain of Beijing, China. Fungal Divers 2007; 25: 69-80

[297] Schulz B, Wanke U, Draeger S, Aust HJ. Endophytes from herbaceous plants and shrubs: effectiveness of surface sterilization methods. Mycol Res 1993; 97: 1447-1450

[298] Fisher PJ. Survival and spread of the endophyte Stagonospora pteridiicola in Pteridium aquilinum, other ferns and some flowering plants. New Phytol 1996; 132: 119-122

[299] Legault D, Dessureault M, Laflamme G. Mycoflore des aiguilles de Pinus banksiana et Pinus resinosa I. Champignons endophytes. Canadian J Bot 1989; 67: 2052-2060

[300] Kharwar RN, Gond SK, Kumar A, Mishra A. A comparative study of endophytic and epiphytic fungal association with leaf of Eucalyptus citriodora Hook. and their antimicrobial activity. World J Microbiol Biotechnol 2010; 26: 1941-1948

[301] Gond SK, Mishra A, Sharma VK, Verma SK, Kumar J, Kharwar RN, Kumar A. Diversity and antimicrobial activity of endophytic fungi isolated from Nyctanthes arbor-tristis, a well-known medicinal plant of India. Mycoscience 2012; 53: 113-121

[302] Rodrigues KF. Fungal Endophytes of Palms. In: Redlin SC, Carris LM, eds. Endophytic Fungi in Grasses and woody Plants: Systematics, Ecology and Evolution. St. Paul, Minnesota, USA: APS Press; 1996: 121-132

[303] Fröhlich J, Hyde KD, Petrini O. Endophytic fungi associated with palms. Mycol Res 2000; 104: 1202-1212

[304] Maria GL, Sridhar KR. Endophytic fungal assemblage of two halophytes from west coastal mangrove habitats, India. Czech Mycol 2003; 55: 241-251

[305] Suryanarayanan TS, Kumaresan V. Endophytic fungi of some halophytes from an estuarine mangrove forest. Mycol Res 2000; 104: 1465-1467

[306] Saikkonen K, Ahlholm J, Helander M, Lehtimäki S, Niemeläinen O. Endophytic fungi in wild and cultivated grasses in Finland. Ecography (Cop.) 2000; 23: 360-366

[307] Thirunavukkarasu N, Suryanarayanan TS, Girivasan KP, Venkatachalam A, Geetha V, Ravishankar JP, Doble M. Fungal symbionts of marine sponges from Rameswaram, southern India: species composition and bioactive metabolites. Fungal Divers 2012; 55: 37-46

[308] Zhang Y, Mu J, Feng Y, Kang Y, Zhang J, Gu PJ, Wang Y, Ma LF, Zhu YH. Broad-spectrum antimicrobial epiphytic and endophytic fungi from marine organisms: isolation, bioassay and taxonomy. Mar Drugs 2009; 7: 97-112

[309] Stone JK, Bacon CW, White JE. An Overview of endophytic Microbes: Endophytism defined. In: Bacon CW, White JF, eds. Microbial Endophytes. New York: Marcel Dekker Inc.; 2000: 3-29

[310] Verma VC, Gond SK, Kumar A, Kharwar RN, Strobel G. The endophytic mycoflora of bark, leaf, and stem tissues of Azadirachta indica A. Juss (neem) from Varanasi (India). Microb Ecol 2007; 54: 119-125 
[311] Mishra A, Gond SK, Kumar A, Sharma VK, Verma SK, Kharwar RN, Sieber TN. Season and tissue type affect fungal endophyte communities of the Indian medicinal plant Tinospora cordifolia more strongly than geographic location. Microb Ecol 2012; 64: 388-398

[312] Gunatilaka AAL. Natural products from plant-associated microorganisms: distribution, structural diversity, bioactivity, and implication of their occurrence. J Nat Prod 2006; 69: 509-526

[313] Verma VC, Gond SK, Kumar A, Mishra A, Kharwar RN, Gange AC. Endophytic actinomycetes from Azadirachta indica A. Juss.: isolation, diversity, and anti-microbial activity. Microb Ecol 2009; 57: 749-756

[314] Kharwar RN, Verma VC, Kumar A, Gond SK, Harper JK, Hess WM, Lobkovosky E, Ma C, Ren Y, Strobel GA. Javanicin, an antibacterial naphthaquinone from an endophytic fungus of neem, Chloridium sp. Curr Microbiol 2009; 58: 233-238

[315] Suryanarayanan TS, Thirunavukkarasu N, Govindarajulu MB, Gopalan V. Fungal endophytes: an untapped source of biocatalysts. Fungal Divers 2012; 54: 19-30

[316] Li X, He X, Hou L, Ren Y, Wang S, Su F. Dark septate endophytes isolated from a xerophyte plant promote the growth of Ammopiptanthus mongolicus under drought condition. Sci Rep 2018; 8: 7896

[317] Jogawat A, Vadassery J, Verma N, Oelmüller R, Dua M, Nevo E, Johri AK. PiHOG1, a stress regulator MAP kinase from the root endophyte fungus Piriformospora indica, confers salinity stress tolerance in rice plants. Sci Rep 2016; 6: 36765

[318] Mastan A, Bharadwaj R, Kushwaha RK, Vivek Babu CS. Functional fungal endophytes in Coleus forskohlii regulate labdane diterpene biosynthesis for elevated forskolin accumulation in roots. Microb Ecol 2019. doi:10.1007/s00248-019-01376-w

[319] Kushwaha RK, Singh S, Pandey SS, Kalra A, Babu CSV. Fungal endophytes attune withanolide biosynthesis in Withania somnifera, prime to enhanced withanolide A content in leaves and roots. World J Microbiol Biotechnol 2019; 35: 20

[320] Ray T, Pandey SS, Pandey A, Srivastava M, Shanker K, Kalra A. Endophytic consortium with diverse gene-regulating capabilities of benzylisoquinoline alkaloids biosynthetic pathway can enhance endogenous morphine biosynthesis in Papaver somniferum. Front Microbiol 2019; 10: 925

[321] Rajagopal K, Suryanarayanan TS. Isolation of endophytic fungi from leaves of neem (Azadirachta indica A. Juss.). Curr Sci 2000; 78: 13751378

[322] Wilson D, Carroll GC. Infection studies of Discula quercina, an endophyte of Quercus garryana. Mycologia 1994; 86: 635-647

[323] Mahesh B, Tejesvi MV, Nalini MS. Endophytic mycoflora of inner bark of Azadirachta indica A. Juss. Curr Sci 2005; 88: 218-219

[324] Verma VC, Singh SK, Prakash S. Bio-control and plant growth promotion potential of siderophore producing endophytic Streptomyces from Azadirachta indica A. Juss. J Basic Microbiol 2011; 51: 550-556

[325] Shao S, Wu S, Chen Y, Wang L, Yang L, Li S, Li Z. Composition of endophytic fungi in Azadirachta indica from Yuanjiang County of Yunnan. Biodivers Sci 2008; 16: 63-67

[326] Tenguria RK, Khan FN. Distribution of endophytic fungi in leaves of Azadirachta indica A. Juss. (Neem) of Panchmarhi Biosphere Reserve. Curr Bot 2011; 2: 27-29

[327] Verma VC, Gond SK, Mishra A, Kumar A, Kharwar RN. Selection of natural strains of fungal endophytes from Azadirachta indica A. Juss, with antimicrobial activity against dermatophytes. Curr Bioact Compd 2008; 4: 36-40

[328] Gohain A, Gogoi A, Debnath R, Yadav A, Singh BP, Gupta VK, Sharma R, Saikia R. Antimicrobial biosynthetic potential and genetic diversity of endophytic actinomycetes associated with medicinal plants. FEMS Microbiol Lett 2015; 362: fnv158

[329] Kuncharoen N, Kudo T, Ohkuma M, Tanasupawat S. Micromonospora azadirachtae sp. nov., isolated from roots of Azadirachta indica A. Juss. var. siamensis Valeton. Antonie Van Leeuwenhoek 2019; 112: 253262

[330] Stierle A, Strobel G, Stierle D. Taxol and taxane production by Taxomyces andreanae, an endophytic fungus of Pacific yew. Science 1993; 260: 214-216

[331] Strobel GA, Miller RV, Miller C, Condron M, Teplow DB, Hess WM. Cryptocandin, a potent antimycotic from the endophytic fungus Cryptosporiopsis cf. quercina. Microbiology 1999; 145: 1919-1926

[332] Li JY, Strobel GA, Harper JK, Lobkovsky E, Clardy J. Cryptocin, a potent tetramic acid antimycotic from the endophytic fungus Cryptosporiopsis cf. quercina. Org Lett 2000; 2: 767-770

[333] Li JY, Harper JK, Grant DM, Tombe BO, Bashyal B, Hess WM, Strobel GA. Ambuic acid, a highly functionalized cyclohexenone with antifungal activity from Pestalotiopsis spp. and Monochaetia sp. Phytochemistry 2001; 56: 463-468

[334] Ding G, Li Y, Fu S, Liu S, Wei ], Che Y. Ambuic acid and torreyanic acid derivatives from the endolichenic fungus Pestalotiopsis sp. J Nat Prod 2009; 72: 182-186

[335] Lee JC, Yang X, Schwartz M, Strobel G, Clardy J. The relationship between an endangered North American tree and an endophytic fungus. Chem Biol 1995; 2: 721-727

[336] Li JY, Strobel GA. Jesterone and hydroxy-jesterone antioomycete cyclohexenenone epoxides from the endophytic fungus Pestalotiopsis jesteri. Phytochemistry 2001; 57: 261-265

[337] Brady SF, Wagenaar MM, Singh MP, Janso JE, Clardy J. The cytosporones, new octaketide antibiotics isolated from an endophytic fungus. Organic Lett 2000; 14: 4043-4046

[338] Singh MP, Janso JE, Brady SF. Cytoskyrins and cytosporones produced by cytospora sp. CR200: taxonomy, fermentation and biological activities. Mar Drugs 2007; 5: 71-84

[339] Lee JC, Strobel GA, Lobkovsky E, Clardy JC. Torreyanic acid: a selectively cytotoxic quinone dimer from the endophytic fungus Pestalotiopsis microspora. J Org Chem 1996; 6: 13232-13233

[340] Zhang LQ, Guo B, Li HY, Zeng SR, Shao H, Gu S, Wei RC. Preliminary study on the isolation of endophytic fungus of Catharanthus roseus and its fermentation to produce products of therapeutic value. Chinese Traditional Herbal Drugs 2000; 31: 805-811

[341] Zhang LQ, Wang HK, Shao H, Shen YM, Zeng SR, Xu CD, Xuan Q Wei RC. Isolation and determination of the anti-cancer substance of chaetoglobosin A producing by endophytic fungus of Maytenus hookeri (in Chinese). Chinese Pharma J 2002; 37: 172-175

[342] Lin Z], Lu ZY, Zhu T], Fang YC, Gu QQ, Zhu WM. Penicillenols from Penicillium sp. GQ-7, an endophytic fungus associated with Aegiceras corniculatum. Chem Pharm Bull 2008; 56: 217-221

[343] Kusari S, Zühlke S, Spiteller M. An endophytic fungus from Camptotheca acuminata that produces camptothecin and analogues. J Nat Prod 2009; 72: 2-7

[344] Harper JK, Ford EJ, Strobel GA, Arif AM, Grant DM, Porco JA jr., Tomer DP, Oneill KK. Pestacin (I): a 1,3-dihydro isobenzofuran from Pestalotiopsis microspora possessing antioxidant and antimycotic activities. Tetrahedron 2003; 59: 2471-2476

[345] Strobel GA, Ford E, Worapong J, Harper JK, Arif AM, Grant DM, Fung PCW, Chan K. Ispoestacin, an isobenzofuranone from Pestalotiopsis microspora, possessing antifungal and antioxidant activities. Phytochemistry 2002; 60: 179-183

[346] Lee J, Lobkovsky E, Pliam NB, Strobel GA, Clardy J. Subglutinols A and B: immunosuppressive compounds from the endophytic fungus Fusarium subglutinans. J Org Chem 1995; 60: 7076-7077

[347] Singh SB, Zink DL, Guan Z, Collado J, Pelaez F, Felock PJ, Hazuda D]. Isolation, structure, and HIV-1 integrase inhibitory activity of xanthoviridicatin $\mathrm{E}$ and $\mathrm{F}$, two novel fungal metabolites produced by Penicillium chrysogenum. Helvetica Chimica Acta 2003; 86: 3380-3385 
[348] Tan RX, Zou WX. Endophytes: a rich source of functional metabolites. Nat Prod Rep 2001; 18: 448-459

[349] Kharwar RN, Mishra A, Gond SK, Stierle A, Stierle D. Anticancer compounds derived from fungal endophytes: their importance and future challenges. Nat Prod Rep 2011; 28: 1208-1228

[350] Chutulo EC, Chalannavar RK. Endophytic mycoflora and their bioactive compounds from Azadirachta indica: a comprehensive review. J Fungi (Basel) 2018; 4: E42

[351] Kusari S, Hertweck C, Spiteller M. Chemical ecology of endophytic fungi: origins of secondary metabolites. Chem Biol 2012; 19: 792-798

[352] Li GH, Yu ZF, Li X, Wang XB, Zheng LJ, Zhang KQ. Nematicidal metabolites produced by the endophytic fungus Geotrichum sp. AL4. Chem Biodivers 2007; 4: 1520-1524

[353] Wu SH, Chen YW, Shao SC, Wang LD, Li ZY, Yang LY, Li SL, Huang R. Ten-membered lactones from Phomopsis sp., an endophytic fungus of Azadirachta indica. J Nat Prod 2008; 71: 731-734

[354] Wu SH, Chen YW, Shao SC, Wang LD, Yu Y, Li ZY, Yang LY, Li SL, Huang R. Two new solanapyrone analogues from the endophytic fungus Nigrospora sp. YB-141 of Azadirachta indica. Chem Biodivers 2009; 6: $79-85$

[355] Wu SH, Chen YW, Shao SC, Li ZZ, Yang LY, Li SL. Studies on the metabolites of Aspergillus aculeatus YM 311498, an endophytic fungus from Azadirachta indica A. Juss. Nat Prod Res Dev 2009; 21: 731-732

[356] Garcia-Sosa K, Villarreal-Alvarez N, Lubben P, Peña-Rodriguez LM. Chrysophanol, an antimicrobial anthraquinone from the root extract of Colubrina greggii. J Mex Chem Soc 2006; 50: 76-78

[357] Kupchan SM, Karim A. Tumor inhibitors. 114. Aloe emodin: antileukemic principle isolated from Rhamnus frangula L. Lloydia 1976; 39: $223-$ 224

[358] Kusari S, Lamshöft M, Zühlke S, Spiteller M. An endophytic fungus from Hypericum perforatum that produces hypericin. I Nat Prod 2008; 71 : 159-162

[359] Wu SH, Chen YW, Miao CP. Secondary metabolites of endophytic fungus Xylaria sp. YC-10 of Azadirachta indica. Chem Nat Comp 2011; 47: 858-861

[360] Boonphong S, Kittakoop P, Isaka M, Pittayakhajonwut D, Tanticharoen M, Thebtaranonth Y. Multiplolides A and B, new antifungal 10-membered lactones from Xylaria multiplex. J Nat Prod 2001; 64: 965-967

[361] Alam SS, Bilton JM, Slawin MZ, Williams DJ, Sheppard RN, Strange RM. Chickpea blight: production of the phytotoxins solanapyrones $A$ and $C$ by Ascochyta rabiei. Phytochemistry 1989; 28: 2627-2630
[362] Hohl B, Weidemann C, Hohl U, Barz W. Isolation of solanapyrones A, B and $C$ from culture filture and spore germination fluids of Ascochyta rabiei and aspects of phytotoxin action. J Phytopathol 1991; 132: 193-206

[363] Latif Z, Strange RN, Bilton ], Riazuddin S. Production of the phytotoxins, solanapyrones $A$ and $C$ and cytochalasin D among nine isolates of Ascochyta rabiei. Plant Pathol 1993; 42: 172-180

[364] Ichihara A, Tazaki H, Sakamura S. Solanapyrones A, B and C, phytotoxic metabolites from the fungus Alternaria solani. Tetrahedron Lett 1983; 24: $5373-5376$

[365] Oikawa H, Yokota T, Sakano C, Suzuki Y, Naya A, Ichihara A. Solanapyrones, phytotoxins produced by Alternaria solani: biosynthesis and isolation of minor components. Biosci Biotechnol Biochem 1998; 62: 2016-2022

[366] Jenkins KM, Toske SG, Jensen PR, Fenical W. Solanapyrones E-G, antialgal metabolites produced by a marine fungus. Phytochemistry 1998; 49: 2299-2304

[367] Wu SH, He J, Li XN, Huang R, Song F, Chen YW, Miao CP. Guaiane ses quiterpenes and isopimarane diterpenes from an endophytic fungus Xylaria sp. Phytochemistry 2014; 105: 197-204

[368] Huang R, Xie XS, Fang XW, Ma KX, Wu SH. Five new guaiane sesquiterpenes from the endophytic fungus Xylaria sp. YM 311647 of Azadirachta indica. Chem Biodivers 2015; 12: 1281-1286

[369] Chatterjee S, Ghosh R, Mandal NC. Production of bioactive compounds with bactericidal and antioxidant potential by endophytic fungus Alternaria alternata AE1 isolated from Azadirachta indica A. Juss. PLoS One 2019; 14: e0214744

[370] Verma VC, Kharwar RN, Gange AC. Biosynthesis of antimicrobial silve nanoparticles by the endophytic fungus Aspergillus clavatus. Nanomedicine (Lond) 2010; 5: 33-40

[371] Kumar J, Sharma VK, Singh DK, Mishra A, Gond SK, Verma SK, Kumar A, Kharwar RN. Epigenetic activation of antibacterial property of an endophytic Streptomyces coelicolor strain azra 37 and identification of the induced protein using MALDI TOF MS/MS. PloS One 2016. doi:10.1371/journal.pone.0147876

[372] Berdy ]. Thoughts and facts about antibiotics: where we are now and where we are heading. J Antibiot 2012; 65: 385-395

[373] Tejesvi VM, Mahesh B, Nalini MS, Prakash HS, Kini KR, Subbiah V, Shetty HS. Fungal endophyte assemblages from ethnopharmaceutically important medicinal trees. Can J Microbiol 2006; 52: 427-435 